\title{
Does Mindfulness Reduce Attentional Negativity Bias and Depressive Symptoms?
}

\author{
Cameron Ford
}

Follow this and additional works at: https://researchrepository.wvu.edu/etd

\section{Recommended Citation}

Ford, Cameron, "Does Mindfulness Reduce Attentional Negativity Bias and Depressive Symptoms?" (2016). Graduate Theses, Dissertations, and Problem Reports. 5609.

https://researchrepository.wvu.edu/etd/5609

This Thesis is protected by copyright and/or related rights. It has been brought to you by the The Research Repository @ WVU with permission from the rights-holder(s). You are free to use this Thesis in any way that is permitted by the copyright and related rights legislation that applies to your use. For other uses you must obtain permission from the rights-holder(s) directly, unless additional rights are indicated by a Creative Commons license in the record and/ or on the work itself. This Thesis has been accepted for inclusion in WVU Graduate Theses, Dissertations, and Problem Reports collection by an authorized administrator of The Research Repository @ WVU. For more information, please contact researchrepository@mail.wvu.edu. 
Does Mindfulness Reduce Attentional Negativity Bias and Depressive Symptoms?

\title{
Cameron Ford, B.A.
}

\author{
Thesis submitted \\ to the Eberly College of Arts and Sciences \\ At West Virginia University \\ in partial fulfillment of the requirements for the degree of \\ Master of Science degree in \\ Psychology
}

Natalie Shook, Ph.D., Chair

Amy Gentzler, Ph.D.

Kevin Larkin, Ph.D.
Department of Psychology
West Virginia University
Morgantown, WV
2016

Keywords: Mindfulness, Negativity Bias, Depression, Eye-tracking Copyright 2016 Cameron Ford 


\begin{abstract}
Does Mindfulness Reduce Attentional Negativity Bias and Depressive Symptoms?

Cameron Ford
\end{abstract}

Mindfulness has been associated with several psychological benefits such as reduced symptoms of depression (Brown, Ryan, \& Creswell, 2007). However, the mechanism(s) by which mindfulness reduces depressive symptoms is unclear. The present research investigated one potential mechanism: attentional negativity bias. In two studies utilizing eye-tracking methodology, gaze patterns served as indicators of visual attention in order to examine the relations among attention, depressive symptoms, and mindfulness. In Study 1, there was some limited evidence that trait mindfulness was related to less attentional negativity bias. Specifically, trait mindfulness was related to more fixation duration and more fixations on positive images. However, there was no evidence that attentional negativity bias mediated the association between mindfulness and depression. In Study 2, state mindfulness was induced in order to test whether a causal relation exists between mindfulness and attentional bias. There were trends such that participants who underwent a state mindfulness induction viewed threat and dysphoric images longer than the control group. There was also some replication of Study 1 findings. Trait mindfulness was associated with less attentional negativity bias; however, trait mindfulness was related to less fixation duration and fewer fixations on negative images. Again, attentional negativity bias did not mediate the mindfulness-depression association. Overall, the studies provide limited and inconsistent evidence that mindfulness is related to less attentional negativity bias and suggest that trait mindfulness and state mindfulness may have differential effects on gaze patterns. 


\section{Table of Contents}

Does Mindfulness Reduce Attentional Negativity Bias and Depressive Symptoms? .............1

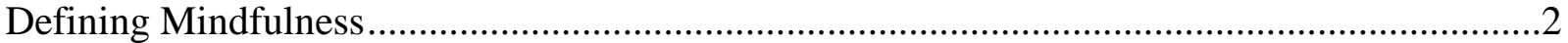

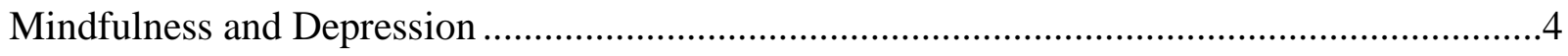

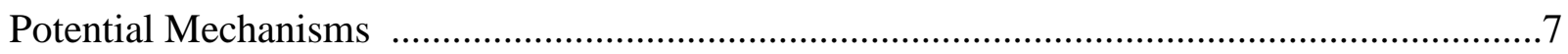

Proposed Mechanism: Attentional Negativity Bias .......................................................9

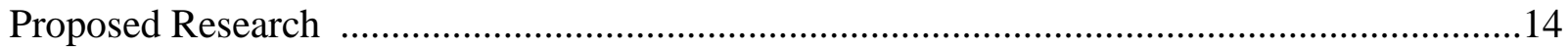

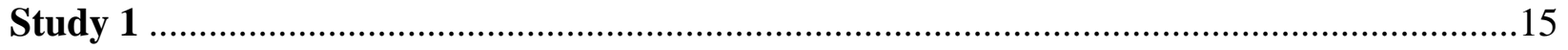

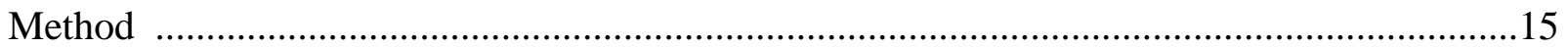

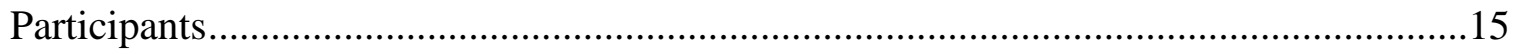

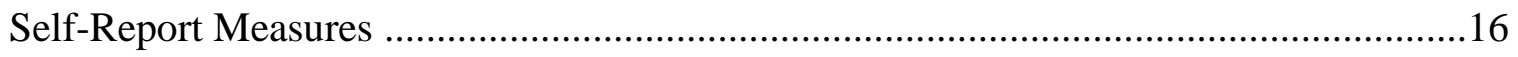

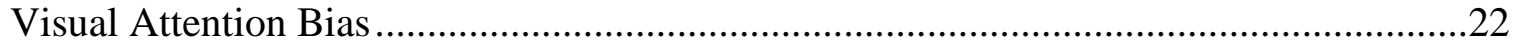

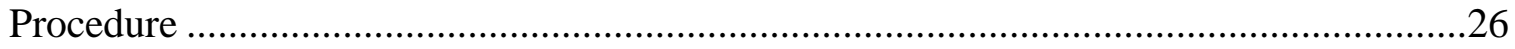

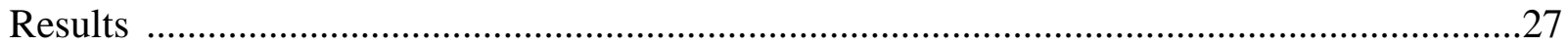

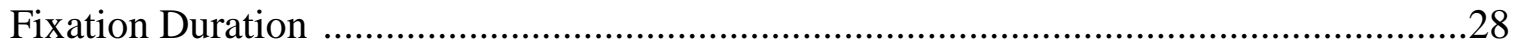

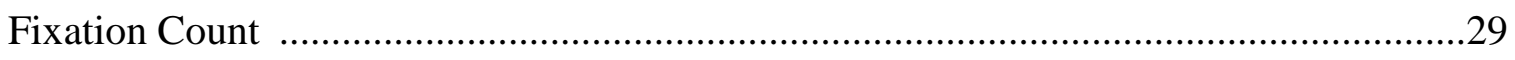

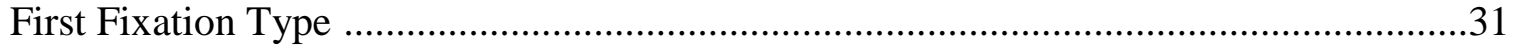

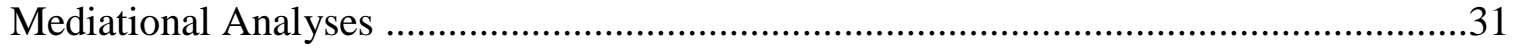

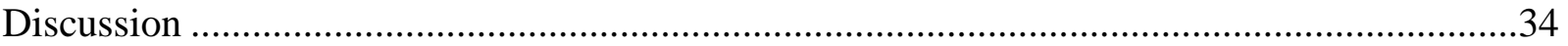

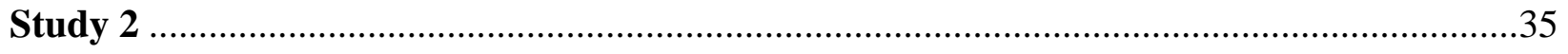

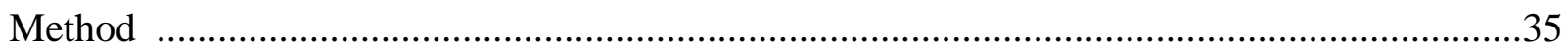

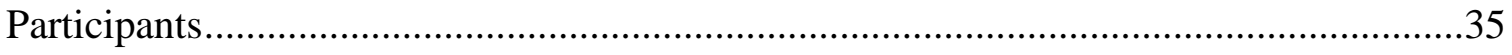

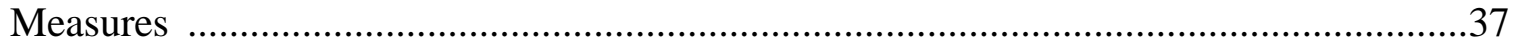

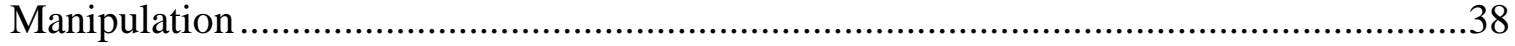

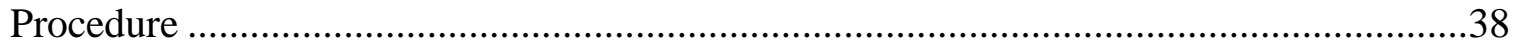

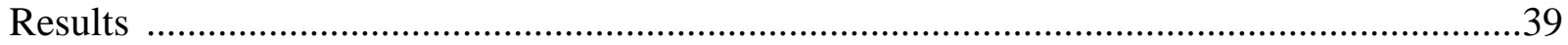

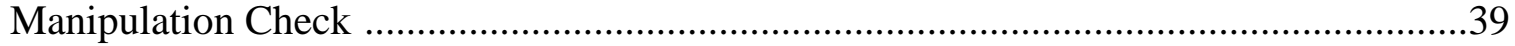

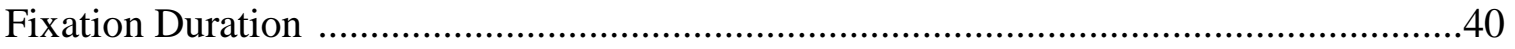

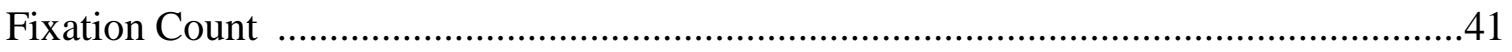

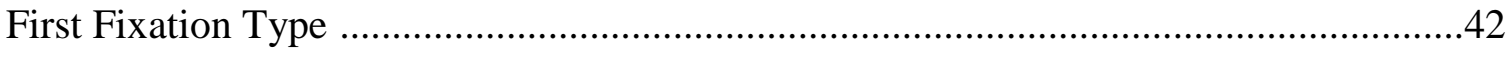

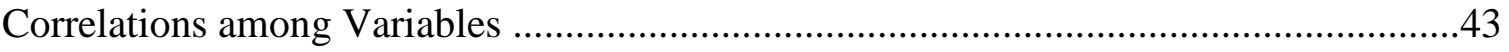

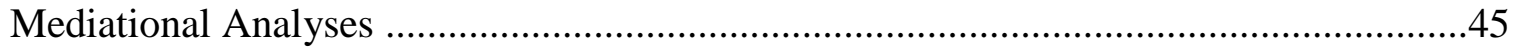




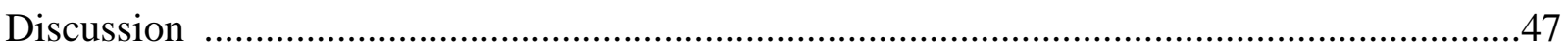

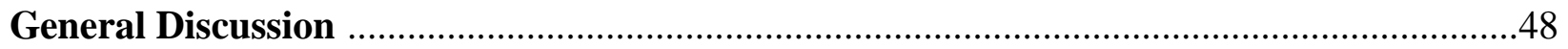

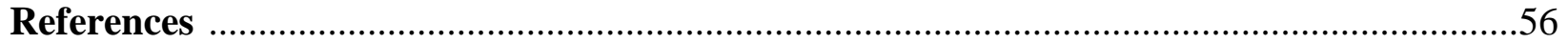

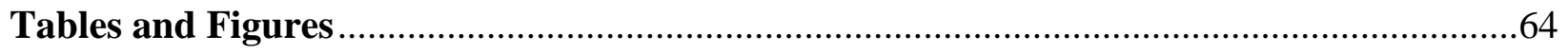

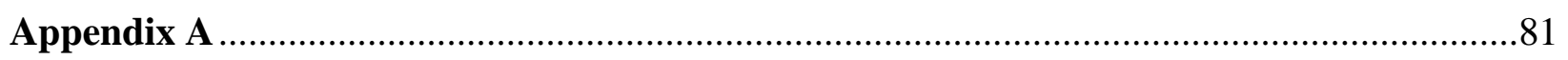

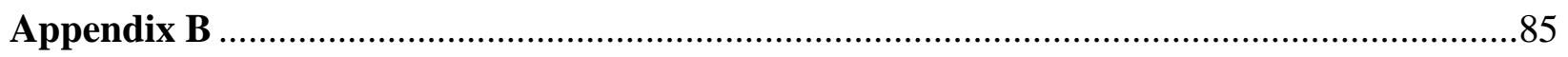

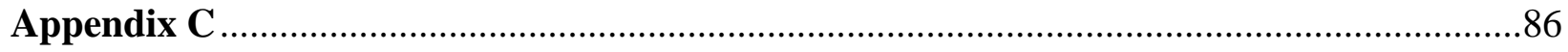


Does Mindfulness Reduce Attentional Negativity Bias and Depressive Symptoms?

Grounded in ancient Buddhist practices, mindfulness involves actively cultivating one’s attention and awareness to the present moment (Brown \& Ryan, 2003). Over the past couple of decades, a growing body of research has demonstrated the benefits of mindfulness for psychological well-being, particularly regarding emotional distress. Mindfulness has been associated with reduced symptoms of depression and anxiety, as well as higher levels of subjective well-being (Brown, Ryan, \& Creswell, 2007). In response to these findings, a number of clinical interventions involving mindfulness practices have been developed to address an array of patient problems (e.g., Acceptance and Commitment Therapy; Hayes, Strosahl, \& Wilson, 1999; Dialectical Behavior Therapy; Linehan, 1993; Mindfulness-Based Cognitive Therapy; Segal, Williams, \& Teasdale, 2002). These mindfulness-based interventions demonstrate clinical utility, especially in reduction of depressive symptoms (e.g., Matthew, Whitford, Kenny, \& Denson, 2010). However, the mechanisms through which mindfulness reduces depressive symptoms have not yet been clearly identified.

The proposed project investigated one possible mechanism: attentional negativity bias. The present study examined whether less attentional negativity bias mediated the inverse relation between mindfulness and depression. In other words, the purpose of the research was to explore whether the association between mindfulness and depression can be explained through visual attentional negativity bias. Utilizing eye-tracking methodology, gaze patterns served as indicators of visual attention in order to examine the relations among attention, depressive symptoms, and mindfulness. The first study was correlational to demonstrate the proposed associations among the variables of interest. In the second study, mindfulness was induced and compared to a mind-wandering control condition in order to investigate whether mindfulness 
affects visual attention and to test whether attentional negativity bias mediated the relation between mindfulness and depressive symptoms. These studies add to the mindfulness literature by examining one potential mechanism by which mindfulness reduces emotional distress.

\section{Defining Mindfulness}

Mindfulness is a sustained, receptive attention to and awareness of internal and external experiences as they occur (Brown \& Ryan, 2003). Kabat-Zinn (1994, p.2) referred to mindfulness as "paying attention in a particular way; on purpose, in the present moment, and non-judgmentally.” Thus, mindfulness is an attention regulation process that involves actively cultivating one’s attention to present moment experiences. Mindfulness stands in contrast to other attentional processes such as rumination, which involves repeatedly focusing attention to one’s emotional distress (Nolen-Hoeksema, Wisco, \& Lyubomirsky, 2008), and worry, which involves focusing attention on uncertain, future events (Borkovec, Ray, \& Stober,1994). Furthermore, Kabat-Zinn (1990) describes mindfulness as focusing attention indiscriminately. That is, observations of present thoughts, feelings, and sensations are experienced as they come and go. The individual is simply an observer of cognitions and does not selectively decide which thoughts to attend to and which thoughts to avoid.

Although researchers have developed several working conceptualizations for mindfulness, one commonality is that most definitions highlight this nonjudgmental, objective perspective. For example, Bucheld, Grossman, and Wallach (2001) maintain that attention to the present must be “without bias or expectation.” Mindfulness requires a willingness to engage with negative, positive, and neutral mind states. Similarly, several self-report scales highlight taking an objective perspective as a critical piece of mindfulness. The Kentucky Inventory of Mindfulness Skill (Baer, Smith, \& Allen, 2004), Cognitive and Affective Mindfulness Scale- 
Revised (Feldman et al., 2007), and the Southampton Mindfulness Questionnaire (Chadwick et al., 2008) all include nonjudgmental perspective or acceptance of mind states as one of the core factors in measuring mindfulness. Thus, mindfulness requires attending to both negative and positive mind states equally in a way that is unbiased and accepting.

Furthermore, mindfulness is conceptualized as both a state of consciousness, as well as a dispositional trait. Kabat-Zinn’s (1990) definition describes mindfulness as a state of paying attention in a particular way. That is, an individual is either in a state of mindfulness or not. A state of mindfulness can be cultivated through regular practice, such as with meditation, yoga, or Tai chi. However, state mindfulness can also be induced in laboratory settings in various ways. For example, mindfulness has been induced via guided self-focused meditation (Broderick, 2005) and guided body scans (Ussher et al., 2014). One of the most commonly used mindfulness inductions is a brief focused breathing audio exercise (Arch \& Craske, 2006) adapted from Kabat-Zinn (1990). The exercise lasts 15 minutes and prompts participants to direct their attention to the actual sensations of the breath without trying to control their breathing. All of these mindfulness inductions instruct the participant to alter their normal attentional process and to direct attention to the present moment.

Mindfulness has also been conceptualized as a dispositional trait. Brown and Ryan (2003) maintain that there are relatively stable differences between people in their propensity to engage in the present moment in an unbiased manner, and that mindful capacity can also be increased or decreased within the individual. Mindfulness based interventions, such as Mindfulness Based Stress Reduction (MBSR; Kabat-Zinn, 1990), generally are intended to increase trait levels of mindfulness by giving individuals the opportunity to enhance mindfulness skills by practicing attending to the present moment. In fact, research has demonstrated that an 
eight-week mindfulness practice increased levels of trait mindfulness (Kiken, Garland, Bluth, Palsson, \& Gaylord, 2015). As such, several self-report measures have been developed to assess trait level mindfulness (e.g., Mindful Attention Awareness Scale, Brown \& Ryan, 2003; Cognitive and Affective Mindfulness Scale - Revised, Feldman, Hayes, Kumar, Greeson, \& Laurenceau, 2007; Five-Facet Mindfulness Questionnaire, Baer et al., 2006).

In sum, mindfulness is an attentional regulation process that involves directing one's attention to whatever is occurring presently without bias or expectation. Whether trying to induce a state of mindfulness or assess trait mindfulness, a primary element of these exercises and measures is attention. Thus, focused attention is a crucial component in defining and engaging in mindfulness.

\section{Mindfulness and Depression}

Mindfulness has been associated with a wide range of physical and psychological health benefits (Brown, Ryan, \& Creswell, 2007; Grossman, Niemann, Schmidt, \& Walach, 2004). For example, mindfulness practice helps to reduce stress and symptoms of chronic pain (Kabat-Zinn, 1982). Of particular importance for this project, a substantial body of research has demonstrated the relation between trait mindfulness and lower levels of emotional distress. Brown and Ryan (2003) found that mindfulness was related to lower levels of emotional disturbance, higher levels of subjective well-being, and higher levels of self-actualization. They also showed that mindfulness was negatively related to symptoms of anxiety and depression. Higher levels of trait mindfulness have also been associated with fewer common cognitive errors, such as forgetting people’s names (Herndon, 2008). Trait mindfulness is also associated with a heightened ability to let go of negative cognitions (Frewen, Evans, Maraj, \& Dozois, 2008). Participants who rated themselves to be higher in dispositional mindfulness tended to self-report a lower frequency of 
negative thoughts and a higher ability to let go of negative thoughts. Taken together, these findings indicate that mindfulness may be useful in addressing symptoms of depression.

Depression is an umbrella term that refers to a number of psychiatric mood disorders, including major depressive disorder (MDD) and persistent depressive disorder (PDD). Prior to the publication of the Diagnostic and Statistical Manual of Mental Disorders, Fifth Edition (DSM-V; American Psychiatric Association, 2013), PDD was previously referred to as Dysthymia. The two hallmark features of depression are low mood and anhedonia, which is the loss of pleasure in previously enjoyable activities. In fact, the DSM-V maintains that an individual must be experiencing at least one of these hallmark symptoms to receive a diagnosis of depression. The DSM-V also lists several other symptoms: significant weight loss or weight gain, insomnia or hypersomnia, psychomotor agitation or retardation, fatigue, feelings of worthlessness or inappropriate guilt, diminished ability to think or concentrate, and recurrent thoughts about death or suicide. Furthermore, depression is also commonly associated with maladaptive cognitive patterns (e.g., Abramson et al., 1989; Beck, 1987). For example, rumination, or repeatedly attending to one’s symptoms of distress (Just \& Alloy, 1997; NolenHoeksema, 2000; Roberts, Gilboa, \& Gotlib, 1998), and dysfunctional attitudes, whereby a person develops negatively biased attitudes about the self, world, and future (Weissman \& Beck, 1978), are often linked with symptoms of depression.

As noted above, trait levels of mindfulness are linked with lower levels of depression (Brown \& Ryan, 2003) and reduced cognitive biases (Frewen, Evans, Maraj, \& Dozois, 2008; Herndon, 2008) as measured by self-report. Some evidence also suggests that interventions utilizing mindfulness practices are useful in alleviating symptoms of emotional distress. More specifically, mindfulness-based interventions can reduce current depressive symptoms 
(Barnhofer et al., 2009; Kenny \& Williams, 2007). Kenny and Williams (2007) found that Mindfulness Based Cognitive Therapy (MBCT) significantly reduced symptoms in a sample of treatment-resistant depressed patients. Furthermore, Barnhofer and colleagues (2009) compared the effectiveness of MBCT and treatment as usual in individuals with at least three previous depressive episodes. They found that patients in the MBCT group experienced a greater reduction in symptoms, and significantly fewer patients in the MBCT condition met criteria for major depressive disorder after treatment compared to the treatment as usual group. Larger randomized controlled experiments have not yet been conducted, but this initial evidence suggests that MBCT can be useful in reducing current symptoms of depression.

Similarly, mindfulness based interventions appear to be useful in decreasing depressive symptoms in individuals with partially remitted symptoms. Specifically, MBCT decreased depressive symptoms measured by the Beck Depression Inventory - II (BDI-II; Beck, Steer, \& Brown, 1996) in a sample of individuals with partially remitted, recurrent unipolar depression compared to a waitlist control group (Britton, Haynes, Fridel, \& Bootzin, 2010). Additionally, in a small sample of 19 participants with residual depressive symptoms, MBCT reduced symptoms significantly more than treatment as usual (Kingston, Dooley, Bates, Lawlor, \& Malone, 2007). Undoubtedly, larger randomized control trials need to be conducted to provide further evidence, but nonetheless preliminary evidence shows mindfulness skills may be beneficial in treating partially remitted symptoms of depression.

In addition to the general harm and dysfunction caused by depression, another problem is that depressive symptoms tend to recur. Approximately 50\% percent of individuals who meet criteria for major depressive disorder will again meet criteria for the disorder within two years after initial recovery (Belsher \& Costello, 1988). Furthermore, each episode of major depression 
increases the risk of recurrence by $18 \%$, and few demographic or personality variables are associated with increased risk of recurrence (Mueller et al., 1999). Some evidence, though, suggests that mindfulness may be useful in preventing relapse of depression, at least in those with multiple depressive episodes. Teasdale and colleagues (2000) found that MBCT significantly reduced major depression recurrence compared to a treatment as usual control group after one year follow-up for individuals who experienced three or more depressive episodes. There was no difference between treatment types for those experiencing fewer than three episodes. Mathew, Whitford, Kenny, and Denson (2010) replicated these findings using a two year follow-up. A recent meta-analysis again found that MBCT significantly reduced risk of recurrence for those with three or more previous episodes, whereas no difference was found for those with two or fewer previous episodes (Piet \& Hougaard, 2011). The authors suggested that relapse in individuals with two or fewer episodes of depression is more often related to significant life events than individuals with three of more episodes, and mindfulness may be less effective at treating depressive episodes resulting from specific life events (Ma \& Teasdale, 2004). Thus, it is possible that MBCT is less effective at preventing relapse spurred by life events.

Together, the growing evidence demonstrates that mindfulness and depression are negatively related and that mindfulness based interventions might be useful in treating depression symptoms, at least in those with chronic-recurrent depression. However, to this point, the mechanism by which these interventions reduce depressive symptoms is not clear. Although a few mechanisms have been proposed and investigated, researchers have not yet agreed upon how exactly mindfulness confers benefits and reduces depression.

\section{Potential Mechanisms}


To date, a few mechanisms have been proposed to explain the association between mindfulness and positive health outcomes. Creswell and Lindsey (2014) offered stress reduction as a possible mechanism of action. The researchers argue that mindfulness reduces physiological stress responses, and this stress reduction is responsible for the change in health outcomes. There is some evidence to support this biological model. Trait mindfulness has been related to lower cortisol reactivity in participants exposed to a high stress situation (Brown, Weinstein, \& Creswell, 2012). Furthermore, mindfulness seems to have its largest effects on high-stress populations, such as patients with psoriasis (Kabat-Zinn et al., 1998). However, there are important limitations in this model. To date, there have been no randomized control trials to determine whether mindfulness training can diminish stress-related responses. Additionally, the model does not stipulate how mindfulness reduces stress responses. It is possible that other factors are contributing to both the diminished stress response and improved outcomes.

Others have proposed enhanced emotion regulation as a mechanism by which mindfulness influences well-being (e.g., Arch \& Craske, 2006; Hayes \& Feldman, 2004). Emotion regulation is the ability for one to respond to present experiences with a flexible range of emotional responses in a way that is socially tolerable (Cole, Michel, \& Teti, 1997). Put another way, emotion regulation involves monitoring, evaluating, and modifying emotional reactions in a way that is consistent with one's goals (Thompson, 1994). A reduced ability to modify emotions can lead to impairment in one's functioning. Rumination provides an example of poor emotion regulation (i.e., inflexibly attending to the symptoms and causes of one's distress). Most support for emotion regulation as a mechanism attempts to establish rumination as a mechanism of action. Indeed, several studies have found a negative relation between mindfulness and rumination (e.g., Brown \& Ryan, 2003; Kiken \& Shook, 2014; Raes \& 
Williams, 2010). Furthermore, two cross-sectional studies found that decreased rumination and enhanced reappraisal mediated the relation between mindfulness and psychological distress (Coffey, Hartman, \& Fredrickson, 2010; Desrosiers, Klemanski, \& Nolen-Hoeksema, 2013). However, there are also limitations with this model. To date, the mediating role of emotion regulation has not been demonstrated in clinical samples of depression. Furthermore, these studies rely solely on self-report instruments, and causality cannot be inferred as these studies are all correlational.

Although evidence for both mechanisms is growing, a broader construct may underlie both models of mindfulness: attentional negativity bias. Attentional negativity bias refers to the tendency for one to selectively focus attention on negative stimuli more so than on positive stimuli (Kellough, Beevers, Ellis, \& Wells, 2008). This bias may provide a more parsimonious model of the mechanism by which mindfulness reduces psychological distress, explaining both stress reduction and emotion regulation models. One of the limitations of the stress response model is that some third variable might be contributing to the reduced stress response and the health benefits. Attentional negativity bias may be that third variable. It is possible that those high in mindfulness simply attend to positive and negative stimuli more equally, and this equanimity could explain the reduced stress response and the health benefits proposed in Creswell and Lindsey’s (2014) model. Similarly, attentional negativity bias can account for the emotion regulation model. Ruminating, or repeatedly attending to one’s emotional distress, may just be a specific example of a broader attentional negativity bias. Thus, attentional negativity bias may provide a more parsimonious model of the mechanism of action in the relation between mindfulness and depression.

\section{Proposed Mechanism: Attentional Negativity Bias}


Cognitive theories of depression maintain that maladaptive, negatively biased thoughts are a key component in depression (Beck, 1987). Accordingly, individuals prone to depression develop negative self-schemata, which in turn result in automatic, negative thoughts about the self, the world, and the future. According to Abramson, Metalsky, and Alloy (1989), these kinds of negative, dysfunctional cognitive styles lead to hopelessness and leave an individual prone to depression. In fact, negatively biased thinking styles predict the first onset and increased lifetime recurrence of depression (Alloy et al., 2006). A recent review suggested that changes in cognitive style after cognitive therapy predict reduction in depressive symptoms (Garratt, Ingram, Rand, \& Sawalani, 2007). Therefore, evidence indicates that negatively biased cognitions increase one’s susceptibility to depression.

More generally, negativity bias refers to the tendency for negative information to weigh more heavily than positive information (for reviews, see Baumeister, Bratslavsky, Finkenauer, \& Vohs, 2001; Rozin \& Royzman, 2001). Negativity bias can be measured cognitively as suggested by the theories above as well as behaviorally. For example, Fazio, Eiser, and Shook (2004) developed a computerized learning task, called BeanFest, which requires participants to learn whether novel stimuli were good or bad. Using healthy college students, initial results demonstrated a learning asymmetry whereby individuals learned negatively valenced stimuli better than they learned positively valenced stimuli. People in general tend to display a negativity bias; however, there is considerable individual variability as to the extent of the negativity bias. In line with cognitive theories of depression, it would logically follow that individuals with depressive symptoms demonstrate a larger negativity bias, and indeed, evidence supports this reasoning. Depressive symptoms and negative cognitive styles have been associated with greater learning asymmetries in the BeanFest task (Conklin, Strunk, \& Fazio, 2009; Shook, Fazio, \& 
Vasey, 2007). That is, individuals with higher levels of depression tended to display larger negativity biases in terms of learning about novel stimuli.

A range of other studies have similarly demonstrated the relation between depressive symptoms and a bias toward negative information compared to positive information. For example, Hindash and Amir (2012) found that individuals reporting depressive symptoms were significantly faster at associating negative words with ambiguous sentences compared to control participants low in depression symptoms. Along the same line, Joorman (2004) found that depressive symptoms were associated with reduced inhibition for negative information. In this study, a non-dysphoric group and a dysphoric group were determined by a median-split on the Center for Epidemiologic Studies Depression Scale. The dysphoric group demonstrated a diminished ability to inhibit negative information. Goeleven, De Raedt, Baert, and Kostner (2006) successfully replicated Joorman's (2004) findings in individuals diagnosed with major depressive disorder. Taken together, the findings are in line with cognitive theories of depression and suggest that individuals with depression have difficulty inhibiting negative information.

Enhanced negativity bias in depression has also been demonstrated with visual attention. Using eye-tracking technology as an indicator of visual attention, numerous studies have found a link between depression and negativity bias in attention. For example, depressive symptoms were associated with increased time spent attending to negative scenes relative to neutral scenes (Caseras, Garner, Bradley, \& Mogg, 2007). In this study, participants were presented with a fixation cross, followed by two images on a screen. One of these images was a neutral scene and the other an emotional scene. The emotional scenes were either positively valenced or negatively valenced. A high dysphoric group, as determined by BDI-II scores greater than the sampled median score of 7, spent a significantly longer time fixating on negative images relative to 
neutral images compared to a low dysphoric group with BDI-II scores lower than the sample median. There was no difference on fixation duration on positive images between groups. A similar study found that dysphoric individuals spent significantly less time directing their visual attention to positive information than a never depressed control group (Sears, Newman, Ference, \& Thomas, 2011). Thus, in two non-clinical samples, higher levels of depressive symptoms were associated with an attentional bias toward negative stimuli and away from positive stimuli.

These relations between depression and attentional negativity bias have also been demonstrated in clinical populations. Isaac and colleagues (2014) found that depressed patients spent significantly less time attending to happy faces compared to non-depressed controls. Kellough, Beevers, Ellis, and Wells (2008) found similar results using an eye-tracking paradigm that included the simultaneous presentation of four stimuli from the International Affective Picture System (IAPS; Lang, Bradley, \& Cuthbert, 2005). On each trial, participants were shown a slide with one image from four different emotion categories: dysphoric, threat, neutral, and positive. The researchers were interested in attentional changes over time and thus presented each slide for thirty seconds. Depressed patients spent significantly more time attending to dysphoric images and less time gazing at positive images than non-depressed participants with no self-reported history of depression. Furthermore, this negativity bias in visual attention was maintained over the course of the thirty seconds. Thus far, the evidence is a bit mixed on whether negativity bias in depression is a result of increased weight given to negative information or reduced weight given to positive information. In either case, the support is convincing that depression involves an increased negativity bias.

Interestingly, mindfulness may be useful in ameliorating the effects of negative cognitive biases. Kabat-Zinn (1994, p.2) referred to mindfulness as "paying attention in a particular way; 
on purpose, in the present moment, and non-judgmentally.” Bucheld, Grossman, and Wallach (2001) also specified that mindful individuals attend to the present without bias or expectation. If an individual is in fact paying attention in a nonjudgmental manner, then all information should theoretically be attended to relatively equally, regardless of valence. Given this definition of mindfulness, it follows that mindfulness may aid in reducing cognitive negativity bias.

Growing evidence supports this reasoning. Kiken and Shook (2011) asked participants to undergo a mind-wandering task or a mindfulness exercise before completing the objective learning task, BeanFest. Individuals induced with state mindfulness demonstrated less negativity bias in learning about novel stimuli than those in the control condition. Similarly, Kiken and Shook (2014) demonstrated that a mindfulness induction reduced the proportion of negative thoughts individuals had in response to emotionally arousing stimuli compared to individuals in a mind-wandering control condition. Furthermore, De Raedt et al. (2011) investigated whether or not negativity bias changed after MBCT. After the 8-week program, individuals with a history of major depressive disorder were better at inhibiting negative information. These studies provide evidence that mindfulness may be effective in reducing negativity bias, as more mindful individuals displayed equality in terms of how they weighed positive and negative information. Thus, it is possible that the automatic response towards negativity bias in depression can be made more flexible with mindfulness. In fact, negatively biased cognitions have been found to partially mediate the relation between mindfulness and emotional distress (Kiken \& Shook, 2012; Gilbert \& Christopher, 2010). These studies, however, were not experimental and thus no causal conclusions could be made.

To the researcher's knowledge, no study has examined the relation between mindfulness and visual attention to date. However, given the demonstrated effects of mindfulness in reducing 
negativity bias, there is reason to believe that mindfulness also reduces visual attention negativity bias. As visual attention negativity bias is associated with depressive symptoms, attentional negativity bias may be the mechanism by which mindfulness has its effects on relieving depressive symptoms. Mindfulness may reduce attentional negativity bias, which in turn may reduce predisposition to depression.

\section{Proposed Research}

The proposed studies investigated whether reduction in negativity bias, specifically negatively biased visual attention, is a mechanism by which mindfulness lessens depression. To do this, two studies were proposed. The first study was correlational to determine the associations among mindfulness, attentional negativity bias, and depression. Mindfulness and depression were measured via self-report measures and visual attention was measured using eyetracking technology. The second study was experimental to test whether attentional negativity bias mediated the relation between mindfulness and depression. Mindfulness was induced via a brief focused breathing exercise. Overall, it was expected that mindfulness reduces depression through visual attention bias. In Study 1, it was hypothesized that mindfulness would be negatively associated with negative visual attention bias and depression. A positive association between attentional negativity bias and depression was expected. Furthermore, it was hypothesized that attentional negativity bias would mediate the relation between mindfulness and endorsement of depressive symptoms. Due to its correlational design, it is important to note that Study 1 did not meet the temporal or causal assumptions of mediation. Thus, for Study 2, the mediation model was tested again, with an experimental manipulation and more closely meeting the temporal assumptions. It was predicted that the mindfulness induction, as compared to a 
control group, would result in less attentional negativity bias, which in turn would reduce endorsement of depressive symptoms.

\section{Study 1}

The purpose of Study 1 was to investigate the relations among the variables of interest. More specifically, the purpose was to establish relations among mindfulness, attentional negativity bias, and depression. Therefore, the design was correlational. It was expected that mindfulness would be negatively associated with both attentional negativity bias and depression. That is, it was predicted that participants higher in mindfulness would exhibit less attentional negativity bias and would report lower levels of depression. It was also anticipated that attentional negativity bias would be positively associated with depression. It was predicted that participants with more of a negativity bias in visual attention would report higher levels of depression. Furthermore, attentional negativity bias was expected to mediate the relation between mindfulness and depression. It was predicted that the association between mindfulness and depression would be explained through reduced attentional negativity bias. Thus, Study 1 addressed four hypotheses: 1) mindfulness measures would be negatively correlated with attentional negativity bias, as assessed by an eye tracking paradigm, 2) mindfulness measures would be negatively correlated with depression measures, 3) visual attentional negativity bias would be positively correlated with depression measures, and 4) attentional negativity bias would mediate the relation between mindfulness and depression.

\section{Method}

\section{Participants}

A total of 166 undergraduate students (75.3\% female; $M_{\text {age }}=19.24$ years, $S D=2.14$, range: 18-41) at West Virginia University were recruited from the Department of Psychology's 
subject pool. To participate, students had to be 18 years or older, fluent English speakers, and not be visually impaired or wear glasses. Thirty-one participants were excluded from statistical analyses due to errors with the eye-tracking task, including failure to properly calibrate participants' gaze, technical difficulties with Tobii eye-tracking software (e.g., software would not open), and research assistants reporting the participant was not engaging in the task. After excluding these participants, outliers were identified. To do this, the total fixation duration on screen times and the ratio of time spent viewing images to viewing the space in between images were converted to z-scores. Scores more than three standard deviations from the mean were excluded. Thus, participants who spent less than $63 \%$ of the task viewing the screen and those who viewed the space in between images $40 \%$ or more of their total time viewing the screen were excluded. Using this criteria excluded an additional ten participants and resulted in a sample of 125 participants (76.0\% female; $M_{\text {age }}=19.33$ years, $S D=2.37$, range: $\left.18-41\right)$. Participants were 82.5\% Caucasian/White, 6.6\% African-American/Black, 6.0\% Asian, 1.2\% Hispanic, 1.2\% Native American, and 2.4\% did not indicate their ethnicity. Participants selfreported considerable engagement with mindfulness-related activities. Sixty-one percent of participants practiced yoga regularly, 39.2\% practiced mediation regularly, 6.0\% practiced tai chi regularly, and 55\% prayed regularly ${ }^{1}$.

\section{Self-Report Measures}

\section{Beck Depression Inventory (BDI - II; Beck, Steer, \& Brown, 1996). To assess} depressive symptoms, the BDI-II was used. This is a 21-item, unidimensional self-report scale that measures depression severity $(\alpha=.93)$. For each item, participants indicate the statement

\footnotetext{
${ }^{1}$ A series of independent samples $t$-tests were conducted to determine whether individuals who practice mindfulness-related activities regularly differ from those who do not on the mindfulness measures. There were no differences between groups, $p s>.05$.
} 
that best describes the way they have felt during the past two weeks, including the day of scale administration. An example item asks participants to describe how often they felt sadness. Participants choose either 0 ("I do not feel sad”), 1 ("I feel sad much of the time”), 2 ("I am sad all of the time”), or 3 (“I am so sad or unhappy that I can’t stand it”). Items are summed to compute a total scale score (Beck, Steer, \& Brown, 1996). Total scores in the 0-13 range indicate minimal depressive symptoms, scores in the 14-19 range indicate mild depressive symptoms, scores in the 20-28 range indicate moderate depressive symptoms, and scores in the 29-63 range indicate severe depressive symptoms. The BDI-II was found to be highly related to the Hamilton Depression Rating with a correlation coefficient of .71 (Beck, Steer, \& Brown, 1996). It also showed a one week test-retest reliability with a correlation coefficient of .93(Beck, Steer, \& Brown, 1996).

Ruminative Response Scale (RRS; Treynor, Gonzalez, \& Nolen-Hoeksema, 2003). Ruminative thinking is repetitive, negative self-focused thinking that is associated with depressive symptoms. Because the current sample consisted of a healthy, undergraduate population, it was anticipated that there would be few BDI-II scores meeting clinical levels of depression and potentially limited variability. Thus, this measure served as an indicator of depression or predisposition for depression (Bagby, Rector, Bacchiochi, \& McBride, 2004). Also, this measure has been used in undergraduate samples with sufficient variability in scores (Roelofs, Muris, Huibers, Peeters, \& Arntz, 2006).

The RRS is a 10-item scale that consists of two subscales: reflection $(\alpha=.72)$ and brooding ( $\alpha=$.77). The reflection subscale (e.g., "If I fail at my work, then I am a failure as a person”) refers to neutrally valenced remembrance of past events, whereas brooding refers to anxiously or moodily pondering the past. Participants indicate the frequency with which they 
engage in various behaviors (e.g., “Think about a recent situation, wishing it had gone better”) on a scale from 1 (“almost never”) to 4 (“almost always”). Items are summed to compute a total subscale score. Higher total scores indicate more reflection or brooding. Both subscales are directly associated with measures of depression and indirectly related to a sense of mastery.

\section{Dysfunctional Attitudes Scale - Short Form 1 (DAS - SF1; Beevers, Strong, Meyer,}

Pilkonis, \& Miller, 2007). Dysfunctional attitudes are negatively biased beliefs about oneself or one's world that are associated with depressive symptoms. For the same reasons as the RRS, the dysfunctional attitudes scale was used as an indicator of depression or a predisposition for depression (Alloy et al., 2006). The DAS - SF1 also shows sufficient variability in scores in undergraduate samples (Beevers et al., 2007).

The DAS - SF1 is a 9-item scale measuring cognitive distortions related to depression ( $\alpha$ $=$.84). Participants indicate how well each statement describes how they generally feel (e.g., "If I fail at my work, then I am a failure as a person”) on a scale from 1 (“totally agree”) to 4 (“totally disagree”). All items on the scale are reverse scored and then summed to compute a total score. Higher total scores indicate more cognitive distortions. The DAS is directly related to other measures of cognitive bias, depression, and hopelessness.

\section{State-Trait Anxiety Inventory (STAI; Spielberger, Gorsuch, Lushene, Vagg, \&}

Jacobs, 1983). Because depression and anxiety are highly comorbid (Regier et al., 1998) and anxiety has been associated with attention bias towards threatening stimuli (Pergamin-Hight, Naim, Bakermans-Kranenburg, van Ijzendoorn, \& Bar-Haim, 2015), it is important to measure anxiety in order to distinguish whether the hypothesized effects relate specifically to depression. Thus, the STAI was included as a potential covariate. This is a 40-item measure of state and trait level anxiety. The inventory consists of two subscales. The first subscale measures state anxiety 
$(\alpha=$.92). Participants indicate how they are currently feeling (e.g., "I am tense") on a scale from 1 ("not at all”) to 4 ("very much so"). The second subscale measures trait anxiety $(\alpha=.92)$. Participants indicate how they generally feel (e.g., I feel nervous and restless) on a scale from 1 ("not at all”) to 4 ("very much so"). Appropriate items on each subscale are reverse scored and then summed to compute total subscale scores. Higher total scores indicate higher levels of anxiety. Both subscales are positively associated with other measures of anxiety, stress, worry, and fear. The STAI demonstrates adequate internal consistency and test-retest reliability at over various time intervals (Barnes, Harp, \& Jung, 2002). As would be expected, the state subscale demonstrates less temporal stability (average $r=.70$ ) than the trait subscale (average $r=.88$; Barnes, Harp, \& Jung, 2002). The STAI has demonstrate strong, positive correlations with the BDI-II (Bieling, Antony, \& Swinson, 1998; Gotlib, 1984).

Beck Anxiety Inventory (BAI; Beck, Epstein, Brown, \& Steer, 1988). In addition to the STAI, the BAI was included as a potential covariate to control for anxiety and ensure that any associations are specific to depression. The BAI is different than the STAI, because the BAI focuses solely on physical symptoms of anxiety. On the other hand, STAI includes only a few items assessing physical symptoms and instead focuses on cognitive symptoms (Julian, 2011). Thus, the BAI was included to assess a different aspect of anxiety. The BAI is a 21-item scale that measures trait anxiety $(\alpha=.88)$. Participants indicate how much they are bothered by common symptoms of anxiety (e.g., unable to relax, difficulty in breathing, hot/cold sweats) on a scale from 0 ("not at all”) to 3 (“severely"). The items are summed to create a total scale, whereby scores less than 21 indicate very low anxiety, scores from 22 to 35 indicate mild anxiety, and scores above 35 indicate severe anxiety. The BAI demonstrated a moderate 
association with the Hamilton Aniexty Rating Scale Revised $(r=.51)$ and a small correlation with the Hamilton Depression Rating Scale ( $r=.25$; Beck, Epstein, Brown, \& Steer, 1988).

\section{Positive and Negative Affect Schedule (PANAS; Watson, Clark, \& Tellegen, 1988).}

This is a 20-item scale that measures state affect. There are two subscales, each consisting of ten items: Positive Affect $(\alpha=.88)$ and Negative Affect $(\alpha=.87)$. Participants indicate the degree to which they are currently experiencing positive (e.g., "excited”," strong, proud) and negative (e.g., distressed, scared, irritable) emotional states on a scale from 1 ("very slightly or not at all”) to 5 ("extremely”). The subscales are weakly correlated, $r=-.17$. This measure was included as a potential covariate to determine whether attentional biases were related to depression above and beyond the effect of negative affect. Items are summed to compute total subscale scores. Higher scores on the positive affect subscale indicate more positive state affect and higher scores on the negative affect subscale indicate more negative state affect. The positive affect subscale $(r=.68)$ and the negative affect subscale $(r=.70)$ demonstrated adequate test-retest reliability over an eight week time period (Watson, Clark, \& Tellegen, 1988). The positive affect subscale was inversely related to the BDI-II ( $r=-.36)$ and the STAI state subscale ( $r=-.35$; Watson, Clark, \& Tellegen, 1988). The negative affect subscale was directly related to the BDI-II $(r=.58)$ and the STAI state subscale ( $r=.51$; Watson, Clark, \& Tellegen, 1988).

Mindful Attention Awareness Scale (MAAS; Brown \& Ryan 2003). This is a 15-item scale that measures attention to and awareness of present moment experience in one's daily life. It is a unidimensional measure of dispositional mindfulness $(\alpha=.87)$. Participants indicate how frequently they have each experience (e.g., I do jobs or tasks automatically, without being aware of what I'm doing) on a scale from 1 (“almost always”) to 6 (“almost never”). All items are reverse coded and then summed to compute a total scale. Higher total scores indicate higher 
levels of trait mindfulness. The MAAS is directly associated with openness to experience $(r=$ .18), internal state awareness ( $r=.23)$, and need for cognition $(r=.19$; Brown \& Ryan 2003). It is also indirectly associated with BDI-II $(r=-.41)$, trait subscale of STAI $(r=-.40)$, and rumination ( $r=.23$; Brown \& Ryan, 2003).

\section{Cognitive and Affective Mindfulness Scale - Revised (CAMS-R; Feldman, Hayes,}

Kumar, Greeson, \& Laurenceau, 2007). This 12-item scale is a unidimensional measure of dispositional mindfulness $(\alpha=.77)$. The scale addresses four components of mindfulness (i.e., acceptance, awareness, attention, and present focus) that load onto a single mindfulness factor. Participants indicate how frequently they have each experience (e.g., "It is easy for me to concentrate on what I am doing.”) on a scale from 1 (“rarely/not at all”) to 4 (“almost always”). The appropriate items are reverse scored and then items are summed to compute a total scale score. Higher total scores indicate greater levels of trait mindfulness. The CAMS-R has been shown to be associated with the MAAS, $r=-.51$ (Feldmen et al., 2007). It is also indirectly related to depression ( $r=-.30)$ and anxiety ( $r=-.24$; Feldmen et al., 2007).

Five Facet Mindfulness Questionnaire (FFMQ; Baer, Smith, Hopkins,

Krietenmeyer, \& Toney, 2006). This 39-item scale measures trait mindfulness. It was developed by administering items from several mindfulness scales and then performing a factor analysis. The FFMQ was an attempt to clarify the essential factors involved with mindfulness. It consists of five subscales: nonreactivity ( $\alpha=.75)$, observing $(\alpha=.83)$, acting with awareness $(\alpha$ $=.87)$, describing $(\alpha=.91)$, nonjudging $(\alpha=.87)$. Participants indicate the degree to which a number of statements (e.g., I find myself doing things without paying attention) are true in their lives on a scale from 1 ("rarely” or "never true”) to 5 ("very often” or “always true”).

Appropriate items are reverse scored, and then items on each subscale are summed to compute a 
total subscale score. Higher scores on each total subscale score indicate higher levels of mindfulness. All of the factors were directly related to psychological well-being, and all but one were associated with meditation experience (Baer et al., 2008). Further, these four subscales mediated the association between meditation experience and well-being.

Demographic Questionnaire. Participants were asked about common demographic variables including age, gender, college rank, sexual orientation, political orientation, marital status, ethnicity, socioeconomic status, and psychology courses taken. Participants were also asked about their experiences with mindfulness-related activities such as yoga, tai chi, or meditation. Furthermore, participants were asked about whether or not they experience color blindness or whether they have specific phobias. These items were included as potential covariates.

\section{Visual Attention Bias}

Apparatus. Visual attention bias was measured by tracking participant gaze patterns. Line of visual gaze was assessed using Tobii X2-30 Eye Tracker Compact Edition hardware and corresponding software (Tobii, Sweden). Tobii X2-30 is an unobtrusive measure of visual gaze and does not require participant head location to be fixed at one location. Participants were situated approximately 26 inches from a computer screen as recommended by the manufacturer specifications. The hardware consisted of one, medium-sized black bar that was attached to the bottom of a computer monitor. This bar was connected to the computer via USB port. The Tobii eye-tracker was connected to a Windows 7 Enterprise 64-bit computer, with 8.00 GB RAM and an i5-2400, 3.10 GHz Intel processor. The eye tracker recorded direction of eye gaze, measured with $x$ and $y$ coordinates, sampling at a rate of $30 \mathrm{~Hz}(S D=2 \mathrm{~Hz})$, with spatial resolution between 0.5 - 1.0 degrees, as per manufacturer specifications. Before the experimental paradigm 
began, there was a calibration process to ensure the gaze pattern indicators were as accurate as possible. Participants were asked to direct their gaze to several fixation points on the screen. Afterwards, the screen displayed where the software tracked the gaze. If the average error of the measured pattern was less than $1^{\circ}$ visual angle from the fixation points, then calibration was complete and the experimental paradigm began. If the measured pattern was inconsistent with the fixation points, calibration was repeated until an error was less than $1^{\circ}$ visual angle. Under ideal conditions, the eye-tracking technology is accurate to .4 degrees, as per manufacturer specifications.

Stimuli. Images from the International Affective Picture System (IAPS; Lang, Bradley, \& Cuthbert, 2005) were used as the stimuli in the eye-tracking paradigm. The IAPS database has been used extensively and the images are well standardized. Each image has been normed for valence and arousal; however, the IAPS images are not categorized by specific emotion. To ensure that each image used in the study represented the desired emotion category, a pilot study was conducted. In this pilot study, undergraduate participants $\left(N=50 ; 70.6 \%\right.$ female; $M_{\text {age }}=$ 19.78 years, $S D=1.98$; $98 \%$ White) were shown images one at a time and then rated the extent to which they presently experienced specific mood states (e.g., happy, sad, scary, disgusting, nervous, and angry) on a scale from 1 (“not at all”) to 5 (“extremely”). Additionally, participants rated each image on a number of other dimensions. They provided ratings of valence ("How pleasant/unpleasant do you feel about the image?”) on a scale from 1 (“extremely unpleasant”) to 9 (“extremely pleasant”), ratings of arousal (“How aroused does this image make you feel?”) on a scale from 1 (“not at all aroused”) to 9 ("very aroused”), and novelty ("How often have you seen the objects or experienced the situations depicted in the image?”) on a scale from 1 (“I’ve never seen or experienced anything like that”) to 9 ("I see or experience these things all the time”). 
Images that uniquely produced feelings of sadness, fear, happiness, or neutrality (i.e., images that did not elicit mixed emotions) were used (see Appendix A for example images).

Four emotional categories (i.e., dysphoric, threat, neutral, and positive) were chosen to demonstrate a general negativity bias (e.g., greater attention to dysphoric or threatening images than positive or neutral images). The threatening images were also included to differentiate depression-related stimuli (e.g., dysphoric images) and anxiety-related stimuli (e.g., threatening images). Symptoms of depression and anxiety are highly comorbid (Regier et al., 1998), and research has demonstrated an attention bias toward threatening stimuli in individuals with anxiety symptoms (Pergamin-Hight, Naim, Bakermans-Kranenburg, van Ijzendoorn, \& BarHaim, 2015). Thus, threatening images were necessary to distinguish whether or not depression is related to a general negativity bias or whether this bias is specific to dysphoric, nonthreatening images.

Participants viewed and rated a total of 80 images. Ultimately, 8 images were chosen for each of the emotion categories: dysphoric, threat, neutral, and positive. Between-group comparisons were conducted using Analysis of Variance (ANOVA) to ensure that the selected images as a group were rated higher than other emotion groups (e.g., to ensure dysphoric images were rated as more sad than other emotion categories). Descriptive and F-test statistics are presented in Table 1. Dysphoric and threat stimuli did not differ on valence or arousal. The dysphoric and threat groups differed from the positive group on valence ratings but not arousal ratings. Furthermore, the images were slightly adjusted using Adobe Photoshop such that the groups did not differ on luminescence, color saturation (red, green, and blue), complexity (e.g., number of pixels in each image) and contrast. The images were matched on these variables because differences between groups potentially affect and confound eye gaze patterns. 
Visual Attention Paradigm. Gaze patterns in an eye-tracking paradigm adapted from Sears and colleagues (2011) were used as indicators of visual attention. In this paradigm, participants were presented with a series of trials in which four images appear simultaneously on a computer screen. Every trial in this eye-tracking paradigm began with a centrally presented fixation cross for three seconds. Then, a slide containing four images was presented for 10 seconds. One image was placed in each of the four distal corners of the slide and were separated by a distance of $4^{\circ}$ on the vertical axis and $8^{\circ}$ on the horizontal axis (see Appendix $\mathrm{B}$ for a sample trial). Participants were instructed to view each image as they naturally would. The task consisted of 24 target trials and 15 filler trials. During target trials, each of the four images represented a different emotion: dysphoric, threatening, neutral, and positive. During filler trials, participants were presented with four neutral images. Filler trials were incorporated to help obscure the purpose of the task. The location of each image was arranged such that each image category occurred in each corner location an equal number of times across the target trials. There were eight images per image category. Thus, each image was presented three times across the target trials. For each repeated presentation, images appeared in a different location and with a different combination of images from the other categories to reduce habituation which could influence eye gaze.

Eye Movement Indices. Three indices of gaze patterns were assessed: fixation duration (FD), fixation count (FC), and first fixation type (FFT) on each image type. A fixation was defined according to an algorithm proposed by Olsson (2007), which is the default setting in Tobii Studio software. Slowly moving gaze patterns (less than .21 pixels per millisecond) constituted a single fixation. Rapidly moving gaze patterns (greater than .21 pixels per millisecond) constituted separate fixation points. Both FD and FC indices were measured by 
Tobii eye tracking software. FD refers to the amount of time a participant fixated upon a given image type. FD was calculated as the ratio of total amount of time spent viewing a particular image category relative to the total amount of time viewing any part of the screen (FD Ratio) ${ }^{2}$. The amount of time a participant fixated upon the black area in between images was measured as well. This made it possible to distinguish when participants were viewing the screen and when participants were looking away from the screen. FC refers to the number of times participants fixated upon each image type. Similar to FD, FC was calculated as the ratio of the number of fixations on a particular image category relative to the total number of fixations on any part of the screen (FC ratio $)^{3}$. First fixation type measures which image type participants first fixated upon. FFT was coded by research assistants. Similar to the method used by Caseras et al. (2008), participants must have been gazing at the center crosshair and then fixated on a particular image for FFT to be recorded. If a participant first fixated on the area between images or looked away from the screen, FFT was not coded. FFT total scores were computed by summing the total number of first fixations on each image type. Attentional negativity bias was operationalized as a tendency to first fixate on the dysphoric stimuli, increased number of fixations on dysphoric stimuli, and increased duration fixated on dysphoric stimuli compared to the other stimuli.

\section{Procedure}

Upon entering the lab, participants were greeted by an experimenter and seated at an individual workstation. The experimenter provided participants with a brief overview of the study. Participants were asked to read and sign informed consent forms. First, participants

\footnotetext{
${ }^{2}$ All analyses were also conducted using the total amount of time participants fixated on a particular image category, instead of FD Ratio. However, similar patterns of results were found, so only FD Ratio results are presented.

${ }^{3}$ All analyses were also conducted using the total number of fixations on a particular image category, instead of FC Ratio. Again, similar patterns of results were found, so only FC Ratio results are presented.
} 
completed the PANAS to measure mood, because mood may be related to visual attention patterns. Next, the experimenter explained the instructions for the eye-tracking paradigm. Participants were told that the eye tracker would measure pupil dilation to obscure the purpose of the task. Participants' gaze was then calibrated with the eye-tracker. After calibration, participants completed 39 trials of the eye tracking paradigm, which took about 10 minutes. Participants were then instructed to complete the questionnaires in the following order: depression scales (BDI-II, RRS, DAS - SF1), mindfulness scales (MAAS, CAMS-R, FFMQ), anxiety scales (BAI, STAI), and finally the demographic questionnaire (see Appendix C for all measures). The primary constructs (e.g., depression and mindfulness) were measured first to minimize participant fatigue. Upon completion of these questionnaires, participants were debriefed, thanked for their participation, granted credit, and excused.

\section{Results}

The distribution plot, as well as skewness and kurtosis, for each measure was observed before statistical analysis. For measures that had a skewness and kurtosis greater than one, log transformations were conducted to normalize the data. FD Ratio for dysphoric images, FC Ratio for dysphoric images, and FC Ratio for the black space between images were transformed. The transformations did not impact the relations among variables. Thus, analyses with untransformed data are presented for ease of interpretation.

Descriptive statistics for all eye-tracking indices are presented in Table 2, and self-report measures with Cronbach's alpha are presented in Table 3. To determine whether mindfulness was associated with depression, Pearson bivariate correlations were conducted between selfreport measures (see Table 4). Generally, all measures of mindfulness were positively correlated, although some subscales of the FFMQ were not significantly related to other measures. 
Additionally, most of the depression-related measures (i.e., BDI, RRS-R, RRS-B, and DAS-SF1) were positively associated with one another. However, the DAS-SF1 and RRS-B were not significantly associated, although the relation was in a positive direction. Overall, mindfulness was inversely associated with depression, rumination, and biased cognition. The CAMSR, MAAS, and four of the five FFMQ subscales were negatively related to BDI. The CAMSR, MAAS, and three of the five FFMQ subscales were negatively related to DAS-SF1; the CAMSR, MAAS, and four of the five FFMQ subscales were negatively related to RRS-R; and the MAAS, and two of the five FFMQ subscales were negatively related to RRS-B.

The measures of anxiety (i.e., STAI-T and BAI) were directly associated. The BAI was significantly related to all depression-related measures. The BAI was also negatively related to the MAAS and three of the five FFMQ subscales. Furthermore, the STAI-T was directly associated with all depression related measures and indirectly associated with all mindfulness measures. Analyses were conducted including measures of anxiety as a covariate. Including BAI as a covariate did not significantly alter the pattern of observed results. Many of the observed correlations between mindfulness and depression were no longer significant after controlling for STAI-T. However, since the effect size of the correlation between STAI-T and BDI was so large, it was concluded the STAI-T was not an adequate measure of anxiety independent of depression. Thus, the presented results do not include any anxiety measures as covariates. Furthermore, negative affect was also not used as a covariate, as down mood is one of the two hallmark symptoms of depression.

\section{Fixation Duration}

To determine whether there were differences in how long participants fixated upon each emotion category, a repeated measures ANOVA with emotion category entered as a within- 
subjects independent variable and FD Ratio as a dependent variable was conducted, $F(3,124)=$ 146.26, $p<.001$. Bonferroni post-hoc analyses revealed that participants viewed dysphoric images longer than any other image type (all $p s<.001)$. Participants viewed positive images longer than threat $(p=.02)$ and neutral images $(p<.001)$, but less than dysphoric images. Participants viewed fear images longer than neutral images $(p<.001)$, but less than dysphoric and positive images.

Bivariate correlations were conducted to determine the relations between FD ratio, mindfulness, and depression (see Table 5). BDI was associated with less time viewing positive images. Additionally, there was a trend such that less time viewing positive images was also related to higher levels of RRS-R, but was unrelated to RRS-B and DAS-SF1. The CAMSR and one of the five subscales of the FFMQ were associated with more time viewing positive images. Furthermore, there was a trend such that the nonreactance FFMQ subscale was positively related to FD ratio on positive images.

To explore negativity bias toward dysphoric images, three difference scores were calculated to compare FD ratio between the dysphoric images and the other image categories. Specifically, FD ratio on positive, fear, and neutral were each subtracted from FD ratio on dysphoric images. Bivariate correlations were conducted to determine the relations between FD ratio difference scores, mindfulness, and depression (see Table 5). BDI and RRS-R were associated with spending more time viewing dysphoric images than positive images. The CAMS-R was associated with spending more time viewing fearful images relative to dysphoric images. Furthermore, the CAMS-R, the FFMQ-observing, and the FFMQ-nonjudging subscale were related to spending more time viewing positive images relative to dysphoric images.

\section{Fixation Count}


To determine whether there were differences in how many times participants fixated upon each emotion category, a repeated measures ANOVA with emotion category entered as a within-subjects independent variable and FC Ratio as a dependent variable was conducted, $F(3$, $124)=201.93, p<.001$. Bonferroni post-hoc analyses revealed that participants fixated on dysphoric images more times than any other image type (all ps <.001). Participants fixated on positive images more times than fear $(p<.001)$ or neutral images $(p<.001)$. Participants fixated on fear images $(p<.001)$ more times than neutral images $(p<.001)$.

Bivariate correlations were conducted to determine the relations between FC ratio mindfulness, and depression (see Table 6). There was a trend such that BDI was associated with fewer fixations on positive images. There was also a trend such that fewer fixations on positive images was also related to RRS-R, but was unrelated to RRS-B and DAS-SF1. Additionally, number of fixations on positive images was directly associated with CAMSR and the FFMQ awareness subscale. There was also a trend such that FFMQ nonreactance subscale was related to more fixations on positive images.

To assess negativity bias, three difference scores were calculated to compare FC ratio between the dysphoric images and other image categories. FC ratio on positive, fear, and neutral images were each subtracted from FC ratio on dysphoric images. Bivariate correlations were conducted to determine the relations between FC ratio difference scores, mindfulness, and depression (see Table 6). PANAS-NA, BDI, and RRS-R were associated with more fixations on dysphoric images relative to positive images. RRS-B and DAS-SF1 were unrelated to all difference scores. The CAMS-R was significantly related to fewer fixations on dysphoric images relative to fearful images. Additionally, there was a trend such that the CAMS-R was related to fewer fixations on dysphoric images relative to positive images. 


\section{First Fixation Type}

To determine whether there were differences in which emotion category participants tended to first fixate, a repeated measures ANOVA with emotion category entered as a withinsubjects independent variable and FFT as a dependent variable was conducted, $F(3,372)=$

33.47, $p<.001$. Bonferroni post-hoc analyses revealed that participants first fixated on dysphoric images more than positive $(p<.001)$ and neutral images $(p<.001)$. Participants did not differ in terms of how often they first fixated on dysphoric and fear images $(p<.001)$. Further, participants did not differ in terms of how often they first fixated on dysphoric and fear images $(p<.001)$

Bivariate correlations were conducted to determine the relations between FFT, mindfulness, and depression (see Table 7). BDI was associated with more first fixations on dysphoric images. RRS-R was also related to more first fixations on dysphoric images, but RRSB and DAS-SF1 were unrelated to first fixation type. The CAMSR was associated with fewer first fixations on dysphoric images. Additionally, there was a trend such that the MAAS and two FFMQ subscales were indirectly related to first fixations on dysphoric images. The FFMQ awareness subscale was the only mindfulness measure directly related to more first fixations on positive images.

\section{Mediation Analyses}

Bivariate correlations replicated previous findings whereby mindfulness was negatively related to depression. Additionally, the results replicated previous research demonstrating that depression was associated with attentional negativity bias. The present study revealed that depression was associated with shorter duration viewing positive images, fewer fixations on positive images, and a tendency to first fixate on dysphoric images. Furthermore, there was some 
limited evidence that mindfulness was associated with decreased attentional bias. Specifically, there was some evidence that mindfulness was associated with more time spent viewing positive images, more fixations on positive images, and less tendency to first fixate on a dysphoric image.

To test whether decreased attentional bias explained the relation between mindfulness and depression, a series of mediation analyses were conducted. Bootstrapping (Preacher \& Hayes, 2004, 2008) was used to examine the direct and indirect effects of mindfulness on depression before and after entering visual attention bias as a mediator. The SPSS INDIRECT macro was used to create 5,000 bootstrap samples and estimate bias-corrected confidence intervals in order to assess the indirect effect of mindfulness on depression (Hayes \& Scharkow, 2013).

First, mediation analyses were conducted to determine whether longer fixation duration on positive images explained the relation between mindfulness and depression. Three mindfulness measures were significantly related to longer fixation duration on positive images: CAMS-R, FFMQ-awareness, and FFMQ-nonreactance. A composite mindfulness score consisting of these three scales was computed. To create a composite score, each scale was standardized, and the standardized scales were averaged. The composite mindfulness score was entered as a predictor variable. FD ratio on positive images was used as a mediator, and BDI was entered as the dependent variable. The indirect effect was -.22, and the 95\% confidence interval ranged from -.81 to .06 . Thus, the indirect effect was not statistically significant. The relation between mindfulness and BDI was not mediated by FD ratio on positive images. An alternative model, whereby mindfulness mediated the relation between FD ratio on positive images and BDI, was also tested. The indirect effect was -16.25 , and the $95 \%$ confidence interval ranged 
from -34.98 to -2.75 . Thus, the indirect effect was statistically significant. The relation between FD ratio on positive images and BDI was mediated by mindfulness.

Second, mediation analyses were conducted to determine whether more fixations on positive images explained the relation between mindfulness and depression. There were two mindfulness measures that were significantly related to more fixations on positive images: CAMS-R and FFMQ-awareness. A composite score using the same method described above was calculated using these two measures. The composite score was entered as a predictor variable. FC ratio on positive images was used as the mediator. BDI was entered as the dependent variable. The indirect effect was -..11, and the 95\% confidence interval ranged from -.51 to .11. Thus, the indirect effect was not statistically significant. The relation between mindfulness and BDI was not mediated by FC ratio on positive images. An alternative model whereby mindfulness mediated the relation between FC ratio on positive images and BDI was also tested. The indirect effect was -20.63 , and the $95 \%$ confidence interval ranged from -42.13 to -3.83 . Thus, the indirect effect was statistically significant. The relation between FC ratio on positive images and BDI was mediated by mindfulness.

Third, mediation analyses were conducted to determine whether reduced tendency to first fixate of dysphoric images explained the relation between mindfulness and depression. CAMS-R was the only mindfulness measure significantly related to FFT on dysphoric images and was entered as the predictor variable. FFT on dysphoric images was used as the mediator. BDI was entered as the dependent variable. The indirect effect was -.30, and the 95\% confidence interval ranged from -1.16 to .05 . Thus, the indirect effect was not statistically significant. The relation between mindfulness and BDI was not mediated by FFT on dysphoric images. An alternative model, whereby mindfulness mediated the relation between FFT on dysphoric images and BDI, 
was also tested. The indirect effect was -.36, and the 95\% confidence interval ranged from .05 to .82. Thus, the indirect effect was statistically significant. The relation between FFT ratio on dysphoric images and BDI was mediated by mindfulness.

\section{Study 1 Discussion}

The purpose of Study 1 was to initially demonstrate the associations among mindfulness, depression, and attentional negativity bias. There was partial support for the first three hypotheses. First, there was mixed evidence that mindfulness was associated with attentional negativity bias. Specifically, some, but not all, measures of mindfulness were associated with less time spent viewing positive images, fewer fixations on positive images, and a reduced tendency to first fixate on a dysphoric image. Additionally, there was a trend such that some measures of mindfulness were related to less fixation duration and fewer fixations on dysphoric images, relative to positive images. Second, there was strong support that mindfulness is negatively associated with depression, which replicated previous findings. Third, depression was related to attentional negativity bias. Specifically, depression was associated with less time spent viewing positive images, fewer fixations on positive images, and a higher probability of first fixating on a dysphoric image.

The fourth hypothesis was not supported. There was no evidence that attentional negativity bias mediated the relation between mindfulness and depression. However, alternate models were tested and supported, whereby mindfulness mediated the relation between attentional negativity bias and depression. That is, less attentional negativity bias was associated with greater trait mindfulness, which in turn, was related to lower levels of depression.

Study 1 consisted of a cross-sectional design; thus, causal claims about the relations between mindfulness and attentional negativity bias cannot be made. Furthermore, the study only 
examined the relations among variables using dispositional, trait measures of mindfulness. Mindfulness can also be conceptualized as a state of being. Although Study 1 revealed that attentional negativity bias did not mediate the relation between dispositional mindfulness and depression, it is possible that the associations among variables may differ when considering state levels of mindfulness.

\section{Study 2}

The purpose of Study 2 was to replicate the associations in Study 1 and to examine whether there was a causal relation between state mindfulness and attentional negativity bias. That is, the study examined whether state mindfulness reduces attentional negativity bias. Further, the proposed mediation model was tested to determine whether the causal relation with attentional negativity bias is the mechanism through which mindfulness has its effect on reducing depression. More specifically, Study 2 involved an experimental manipulation of mindfulness to investigate whether attentional negativity bias mediated the relation between state mindfulness and depression. The experimental group engaged in a mindful breathing task, and the control group engaged in a mind-wandering task. It was expected that participants randomly assigned to the mindful breathing task would demonstrate less of a negativity bias in visual attention than participants assigned to the mind-wandering condition, and that this reduction in attention bias would explain the negative association between mindfulness and depression. Thus, it was hypothesized that 1) mindfulness would reduce attentional negativity bias and 2) that attentional negativity bias would mediate the relation between mindfulness and depression.

\section{Method}

\section{Participants}


A total of 164 undergraduate students (75.6\% female; $M_{\text {age }}=19.34$ years, $S D=2.15$, range: 18-33) at West Virginia University were recruited from the Department of Psychology's subject pool. To participate, students had to be 18 years or older, fluent English speakers, and not be visually impaired or wear glasses. Eleven participants were excluded from statistical analyses due to errors with the eye-tracking task, including failure to properly calibrate participants' gaze, technical difficulties with Tobii eye-tracking software (e.g., software would not open), and research assistants reporting the participant was not engaging in the task. After excluding these participants, outliers were identified. The same criteria used to determine outliers in Study 1 were used in Study 2. Thus, participants who spent less than $63 \%$ of the task viewing the screen and those who viewed the space in between images $40 \%$ or more of their total time viewing the screen were excluded. Using this criteria excluded an additional twenty participants and resulted in a sample of 123 participants $\left(78.9 \%\right.$ female; $M_{\text {age }}=19.54$ years, $S D=2.16$, range: $\left.18-33\right)$. Participants were 86.2\% Caucasian/White, 3.3\% African-American/Black, 2.4\% Asian, 1.6\% Hispanic, and 6.5\% indicated their ethnicity as "other."

Participants self-reported considerable engagement with mindfulness-related activities: 73.2\% of participants had previously practiced yoga, 50.4\% had previously practiced meditation, 6.5\% had previously practiced tai chi, and 33.3\% prayed regularly. A series of independent samples $t$-tests were conducted to determine whether individuals who practiced mindfulnessrelated activities regularly differed from those who did not on any of the mindfulness measures. Individuals who had previously practiced yoga were higher in trait level mindfulness than individuals with no yoga experience ${ }^{4}$. Specifically, individuals with experience doing yoga were higher on the MAAS, $t(119)=2.10, p=.038$, and FFMQ-nonjudging, $t(119)=2.55, p=.01$,

\footnotetext{
${ }^{4}$ Controlling for yoga experience did not alter the pattern of results from the reported analyses.
} 
than those who did not have any prior experience with yoga. There were no differences between all other groups, $p s>.05$.

\section{Measures}

Participants completed the same measures as described in Study 1. This includes the Beck Depression Inventory - II, Rumination Response Scale, Dysfunctional Attitudes Scale Short Form II, State-Trait Anxiety Inventory, Beck Anxiety Inventory, Positive and Negative Affect Schedule, Mindful Attention Awareness Scale, Cognitive and Affective Mindfulness Scale - Revised, Five Facet Mindfulness Scale, and demographic questions. Attentional negativity bias using the eye-tracking paradigm was assessed exactly the same way as in Study 1. A couple of measures were included in Study 2 as manipulation checks.

State Mindful Attention Awareness Scale (State MAAS; Brown and Ryan, 2003). The State MAAS was used as a manipulation check to determine whether the mindfulness induction condition resulted in higher levels of state mindfulness relative to the control mind wandering condition. This 5-item scale is a unidimensional measure of state mindfulness ( $\alpha=$ .92). Participants indicate the degree to which they had several experiences during a task (e.g., I was finding it difficult to stay focused on what was happening) on a scale from 0 (“not at all”) to 6 ("very much”). All items on the scale are reverse scored and then summed to create a total scale score. Higher total scores indicate higher state mindfulness. The State MAAS has been shown to predict trait MAAS scores, $B=.46, t=4.47, p<.0001$ (Brown and Ryan, 2003). Additionally, the State MAAS also predicts high positive affect and lower negative affect above and beyond trait levels of mindfulness (Brown and Ryan, 2003).

Mood Questionnaire (Mata, Hogan, Joorman, Waugh \& Gibb, 2013). The mood questionnaire was included to ensure that the mindfulness induction did not result in a different 
mood state than the mind-wandering control condition. Using a 7-point Likert-type scale from 1 (not at all) to 7 (very), participants are asked to respond to two questions: "How positive are you feeling right now?” and “How negative are you feeling right now?” Responses to the two items are indicators of positive affect and negative affect, respectively.

\section{Manipulation}

Participants were randomly assigned using a random number generator in Excel to either a mindfulness induction condition or a mind-wandering control condition. In the mindfulness induction condition, participants were instructed through a mindful breathing exercise (Kiken \& Shook, 2011), which was adapted from Arch and Craske (2006). Participants were guided to focus their attention to each breath as it occurs. Additional instructions encouraged participants to briefly acknowledge any thoughts or feelings and then direct attention back to their breath. Variations of these instructions were repeated for 15 minutes. A mind-wandering condition served as a control condition (Kiken \& Shook, 2011). In the mind-wandering condition, participants were instructed to let their mind wander freely. Participants were guided to think about whatever comes to mind without focusing on anything in particular. Variations of these instructions were repeated for 15 minutes. The audio instructions for each condition were standardized for length of exercise, length of instructions, and time interval between instructions.

\section{Procedure}

The procedure for Study 2 was similar to the procedure for Study 1. The key difference in procedure was that participants underwent either a mindfulness induction or a mind-wandering task before the eye tracking paradigm. Upon entering the lab, participants were greeted by an experimenter and seated at an individual workstation. The experimenter provided participants with a brief overview of the study. Participants were asked to read and sign informed consent 
forms. Participants were randomly assigned to either the mindfulness induction condition or the mind-wandering condition. Then, they were guided through the respective exercise. Both exercises lasted approximately 15 minutes. After the exercise, participants completed the mood questionnaire and the state MAAS. Next, the experimenter explained the instructions for the eye tracking paradigm. Participants’ gaze was then calibrated with the eye-tracker. After calibration, participants completed 39 trials of the eye tracking paradigm. Participants were then instructed to complete the questionnaires in the following order: depression scales (BDI-II, RRS, DAS - SF1), mindfulness scales (MAAS, CAMS-R, FFMQ), anxiety scales (BAI, STAI), and finally the demographic questionnaire. Upon completion of these questionnaires, participants were thanked for their participation, granted credit, and excused.

\section{Results}

The distribution plot, as well as skewness and kurtosis, for each measure was observed before statistical analysis. For measures that had a skewness and kurtosis greater than one, log transformations were conducted to normalize the data. FD Ratio for the black space between images, FC Ratio for the black space between images, and PANAS-NA were transformed. The transformations did not impact the relations among variables. Thus, analyses with untransformed data are presented for ease of interpretation. Descriptive statistics split by condition for all eyetracking indices are presented in Table 8, and self-report measures with Cronbach's alpha are presented in Table 9.

\section{Manipulation Check}

Before testing the primary hypotheses, a manipulation check was conducted to investigate whether the mindfulness induction resulted in more state mindfulness relative to the mind-wandering task. An independent samples $t$-test on state MAAS scores revealed that there was no difference in state mindfulness between groups, $t(119)=0.22, p=.83$. Additionally, two 
independent samples $t$-test were conducted on the mood questionnaire items between the experimental and control conditions. The two groups did not differ on positive mood, $t(119)=$ $0.69, p=.59$, or negative mood, $t(119)=-0.54, p=.59$.

Furthermore, a series of independent $t$-tests and chi square analyses were conducted to determine if the two groups differed on other demographic items. The two groups did not differ in the number of females, $X^{2}(2, \mathrm{~N}=121)=.99, p=.61$, ethnicity, $X^{2}(3, \mathrm{~N}=114)=.1 .34, p=$ .72 , number of individuals who had previous experience with yoga, $X^{2}(1, \mathrm{~N}=120)=.39, p=$ .68 , number of individuals who had previous experience with meditation, $X^{2}(1, \mathrm{~N}=120)=.03, p$ $>$.99, number of individuals who had previous experience with tai chi, $X^{2}(1, \mathrm{~N}=120)=2.14, p$ $=.27$, or number of individuals who pray often, $X^{2}(1, \mathrm{~N}=120)=.55, p=.58$. Additionally, the two groups did not significantly differ in BDI, $t(119)=0.64, p=.52$, DAS-SF1, $t(119)=0.35, p$ $=.73, \mathrm{RRS}-\mathrm{R}, t(119)=0.95, p=.34, \mathrm{RRS}-\mathrm{B}, t(119)=0.64, p=.52, \mathrm{STAI}-\mathrm{S}, t(119)=0.04, p=$ .97, STAI-T, $t(119)=0.55, p=.58$, BAI, $t(119)=-0.11, p=.91$, PANAS - positive affect, $t(119)$ $=-0.35, p=.73$, or PANAS - negative affect, $t(119)=-.21, p=.83$.

Although the two conditions did not differ in state mindfulness, the State MAAS demonstrated poor reliability in this sample $(\alpha=.63)$. As such, it is unclear whether the manipulation had an effect or not. Thus, the initial analytic strategy was pursued.

\section{Fixation Duration}

To determine whether the manipulation caused changes in gaze patterns, a 2 (condition) by 4 (image type) mixed-factorial ANOVA was conducted with FD Ratio as the dependent variable. Mauchly's Test of Sphericity indicated that the assumption of sphericity had been violated, $\chi^{2}(5)=0.79, p<.001$. A Greenhouse-Geisser Correction was used to combat the violation of the assumption of sphericity. There was a significant main effect of image type, 
$F(2.60,309.81)=135.87, p<.001$. Post hoc pairwise comparisons revealed that participants viewed dysphoric images longer than all other image types (all $p s<.01$ ), positive images more than threat $(p<.001)$ and neutral images $(p<.001)$, and threat images longer than neutral images $(p<.001)$. There was no main effect of condition, $F(1,119)=.97, p=.33$. There was also a significant condition by image type interaction, $F(2.60,309.81)=2.74, p=.05$ (see Figure 1 ). To probe the interaction, simple effects analyses were performed. To reduce the probability of a Type I error, $p$ value adjustments were made using the Least Significant Difference. The simple effect analyses revealed that the mindfulness group viewed threat images longer than the mindwandering group, $p=.03$. Furthermore, there was a trend such that the mindfulness group viewed dysphoric images longer than the mind-wandering group, $p=.10$.

As in Study 1, to explore negativity bias toward dysphoric images, three difference scores were calculated to compare FD ratio between the dysphoric image and the other image categories. Specifically, FD ratio on positive, fear, and neutral were each subtracted from FD ratio on dysphoric images. To determine whether the manipulation caused changes in these difference scores, three independent samples $t$-tests were conducted. There was a trend such that the difference between FD on dysphoric images and FD on positive images was greater in the mindfulness group, $t(119)=-1.84, p=.07$. Furthermore, there was a trend such that the difference between FD on dysphoric images and FD on neutral images was greater in the mindfulness group, $t(119)=-1.73, p=.09$. The two groups did not differ on FD on dysphoric images relative to FD on threat images, $t(119)=0.14, p=.89$.

\section{Fixation Count}

To determine whether the manipulation caused changes in gaze patterns, a 2 (condition) by 4 (image type) mixed-factorial ANOVA was conducted on FC Ratio. Mauchly's Test of 
Sphericity indicated that the assumption of sphericity had been violated, $\chi^{2}(5)=0.84, p=.001$. A Greenhouse-Geisser Correction was used to combat the violation of the assumption of sphericity. There was a significant main effect of image type, $F(2.70,320.67)=161.52, p<.001$. Post hoc pairwise comparisons revealed that participants fixated on dysphoric images more than on any other image type (all $p$ s $<.001)$, fixated on positive images more than threat $(p<.001)$ and neutral images $(p<.001)$, and fixated on threat images more than neutral images $(p<.001)$. There was no main effect of condition, $F(1,119)=.52, p=.47$. There was not a significant condition by image type interaction, $F(2.70,320.67)=2.03, p=.12$.

As in Study 1, to explore negativity bias toward dysphoric images, three difference scores were calculated to compare FC ratio between the dysphoric image and the other image categories. Specifically, FC ratio on positive, fear, and neutral, were each subtracted from FC ratio on dysphoric images. To determine whether the manipulation caused changes in these difference scores, three independent samples $t$-tests were conducted. The two groups did not differ on FD on dysphoric images relative to FD on threat images, $t(119)=0.31, p=.76$, FD on dysphoric images relative to FD on positive images, $t(119)=-1.34, p=.19$, or FD on dysphoric images relative to FD on neutral images, $t(119)=-1.63, p=.11$.

\section{First Fixation Type}

To determine whether the manipulation caused changes in gaze patterns, a 2 (condition) by 4 (image type) mixed-factorial ANOVA was conducted on FFT. Mauchly's Test of Sphericity indicated that the assumption of sphericity had been violated, $\chi^{2}(5)=0.80, p<.001$. A Greenhouse-Geisser Correction was used to combat the violation of the assumption of sphericity. There was a significant main effect of image type, $F(2.60,309.29)=13.09, p<.001$. Post hoc pairwise comparisons revealed that participants first fixated on dysphoric images more than on 
any other image type (all ps <.01). Participants did not differ in how often they first fixated on positive, threat, or neutral images (all $p s>.10)$. There was no main effect of condition, $F(1,119)$ $=.02, p=.89$. There was not a significant condition by image type interaction, $F(2.60,309.29)=$ $.86, p=.46$.

\section{Correlations among Variables}

Self-Report. Bivariate correlations among all self-report measures were conducted (see Table 10). Generally, all measures of trait mindfulness were positively correlated, although some subscales of the FFMQ were not significantly related to other measures. Additionally, most of the depression-related measures (i.e., BDI, RRS-R, RRS-B, and DAS-SF1) were positively associated with one another. However, the DAS-SF1 and RRS-B were not significantly associated, although there was a trend such that the two were directly associated. Overall, trait mindfulness was indirectly associated with depression, rumination, and biased cognition. The CAMSR, MAAS, and four of the five FFMQ subscales were negatively related to BDI. The CAMSR, MAAS, and four of the five FFMQ subscales were negatively related to DAS-SF1; the CAMSR, MAAS, and three of the five FFMQ subscales were negatively related to RRS-R; and the MAAS, and two of the five FFMQ subscales were negatively related to RRS-B.

Additionally, the measures of anxiety (i.e., STAI-S, STAI-T, and BAI) were directly associated. BAI was significantly related to all depression-related measures. The BAI was also negatively related to CAMSR, MAAS, and three of the five FFMQ subscales. Furthermore, the STAI-S and STAI-T were directly associated with all depression related measures and indirectly associated with the CAMSR, MAAS and four of the five FFMQ subscales.

Partial correlations were conducted between the self-report measures and the eye-tracking measures, controlling for BAI. The overall pattern of results was generally the same when 
controlling for BAI. Since the effect size of the correlation between STAI-T, STAI-S, and BDI, and were so large, the measures seemed to have been measuring very similar constructs. Thus, the presented results do not include any anxiety measures as covariates. Furthermore, negative affect was also not used as a covariate, as down mood is one of the two hallmark symptoms of depression. Partial correlations were conducted between the self-report measures and the eyetracking measures, controlling for condition as well. Including condition as a covariate did not significantly influence the pattern of results. Thus, the presented results do not include any anxiety, mood, or condition as covariates.

FD ratio. Bivariate correlations were conducted to determine the relations between FD ratio, trait mindfulness, and depression to determine whether the associations found in Study 1 were replicated in Study 2 (see Table 11). There were trends such that BDI and DAS-SF1 were associated with more time viewing dysphoric images. RRS-R and RRS-B were unrelated to FD Ratio scores. The FFMQ describing subscale was associated with less time viewing dysphoric images. Additionally, there was a trend such that the MAAS was associated with less time viewing dysphoric images.

As in Study 1, to explore negativity bias toward dysphoric images, three difference scores were calculated to compare FD ratio between the dysphoric images and the other image categories. Specifically, FD ratio on positive, fear, and neutral were each subtracted from FD ratio on dysphoric images. Bivariate correlations were conducted to determine the relations between FD ratio difference scores, trait mindfulness, and depression (see Table 11). BDI was associated with spending more time viewing dysphoric images than neutral images. DAS-SF1, RRS-R, and RRS-B were unrelated to difference scores. Although none of the mindfulness measures were significantly related to difference scores, there was a trend such that MAAS and 
FFMQ describing subscale were associated with less time viewing dysphoric images relative to neutral images.

FC ratio. Bivariate correlations were conducted to determine the relations between FC ratio, trait mindfulness, and depression (see Table 12). BDI was associated with more fixations on dysphoric images. There were trends such that DAS-SF1 and RRS-B were related to more fixations on dysphoric images, whereas the RRS-R was unrelated to FC. The MAAS was associated with fewer fixations on dysphoric images. There were trends such that the CAMSR and two FFMQ subscales were also related with fewer fixations on dysphoric images.

As in Study 1, to assess negativity bias, three difference scores were calculated to compare FC ratio between the dysphoric images and other image categories. FC ratio on positive, fear, and neutral images were each subtracted from FC ratio on dysphoric images. Bivariate correlations were conducted to determine the relations between FC ratio difference scores, trait mindfulness, and depression (see Table 12). BDI was associated with more fixations on dysphoric images than neutral images. The MAAS was significantly related to fewer fixations on dysphoric images than neutral images. Additionally, there was a trend such that three FFMQ subscales were related to fewer fixations on dysphoric images than neutral images.

FFT. Bivariate correlations were conducted to determine the relations between FFT, trait mindfulness, and depression (see Table 13). BDI, RRS-R, and DAS-SF1 were unrelated to all FFT measures. RRS-B was related to more first fixations on dysphoric images and more first fixations on positive images. The FFMQ observing subscale was associated with fewer first fixations on threat images, and there was a trend that FFMQ observing was also related to fewer first fixations on dysphoric images.

\section{Mediation Analyses}


Because the series of mixed model ANOVAs revealed that the mindfulness induction did not reduce attentional negativity bias, there was not sufficient grounds to test the proposed mediation model $^{5}$.

As in Study 1, mediation analyses were conducted to test whether the decreased attentional bias explained the relation between trait mindfulness and depression. Bootstrapping (Preacher \& Hayes, 2004, 2008) was used to examine the direct and indirect effects of mindfulness on depression before and after entering visual attention bias as a mediator. The SPSS INDIRECT macro was used to create 5,000 bootstrap samples and estimate bias-corrected confidence intervals in order to assess the indirect effect of mindfulness on depression (Hayes \& Scharkow, 2013).

First, mediation analyses were conducted to determine whether shorter fixation duration on dysphoric images explained the relation between trait mindfulness and depression. FFMQ describing subscale was the only trait mindfulness measure significantly related to shorter fixation duration on dysphoric images. FFMQ describing subscale was entered as a predictor variable. FD ratio on dysphoric images was used as a mediator, and BDI was entered as the dependent variable. The indirect effect was -.02, and the 95\% confidence interval ranged from -.11 to .01. Thus, the indirect effect was not statistically significant. The relation between mindfulness and BDI was not mediated by FD ratio on dysphoric images. An alternative model, whereby mindfulness mediated the relation between FD ratio on dysphoric images and BDI, was also tested. The indirect effect was 6.62 , and the 95\% confidence interval ranged from .22 to 18.15. Thus, the indirect effect was statistically significant. The relation between FD ratio on dysphoric images and BDI was mediated by mindfulness.

\footnotetext{
${ }^{5}$ Moderation analyses were also conducted to investigate whether trait mindfulness or depression moderated the effect of the manipulation on gaze patterns. These analysis did not reveal evidence for moderation, $p s>.05$.
} 
Second, mediation analyses were conducted to determine whether fewer fixations on dysphoric images explained the relation between mindfulness and depression. MAAS was the only mindfulness measures that was significantly related to fewer fixations on dysphoric images. MAAS was entered as a predictor variable. FC ratio on dysphoric images was used as the mediator. BDI was entered as the dependent variable. The indirect effect was -.26, and the 95\% confidence interval ranged from -1.00 to .05 . Thus, the indirect effect was not statistically significant. The relation between mindfulness and BDI was not mediated by FC ratio on dysphoric images. An alternative model whereby mindfulness mediated the relation between FC ratio on positive images and BDI was also tested. The indirect effect was 17.76 , and the $95 \%$ confidence interval ranged from 2.17 to 37.62 . Thus, the indirect effect was statistically significant. The relation between FC ratio on dysphoric images and BDI was mediated by mindfulness.

Mediation analyses were not conducted with FFT, because FFT was unrelated to all depression-related measures.

\section{Study 2 Discussion}

The purpose of Study 2 was twofold. First, the study was designed to determine whether the relation between state mindfulness and attentional negativity bias was causal. The results did not support this hypothesis. In fact, the results demonstrated some limited evidence that a mindfulness induction, relative to a mind-wandering induction, increased the amount of time participants viewed dysphoric and threat images. That is, the brief mindfulness induction may have increased attentional negativity bias. All other measures of attentional negativity bias did not differ between groups. These results are taken with caution, though. Because the State 
MAAS demonstrated poor reliability in this sample, it is unclear whether the manipulation had an effect or not.

Second, Study 2 attempted to replicate the associations among attentional negativity bias, mindfulness, and depression. Study 2 revealed an inverse relation between mindfulness and depression. Depression was also associated with more time viewing and more fixations on dysphoric images. There was some evidence that mindfulness was associated with less time viewing and fewer fixations on dysphoric images. As in Study 1, there was no evidence that attentional negativity bias mediated the relation between mindfulness and depression. However, alternate models were tested and supported, whereby mindfulness mediated the relation between attentional negativity bias and depression. That is, less attentional negativity bias was associated with greater trait mindfulness, which in turn, was related to lower levels of depression.

\section{General Discussion}

The purpose of this research was to determine whether less attentional negativity bias was a mechanism by which mindfulness reduces depression. It was predicted that attentional negativity bias would mediate the relation between mindfulness and depression. Study 1 was a correlation design that tested the hypothesized associations between variables, and Study 2 was designed to test whether a causal relation existed between state mindfulness and attentional negativity bias. Across both studies, there was some indication that trait mindfulness was associated with reduced attentional negativity bias, but the evidence was limited and inconsistent. The proposed mediation model was tested and results revealed that attentional bias did not mediate the relation between mindfulness and depression. Furthermore, Study 2 demonstrated that a brief mindfulness induction increased the amount of time participants viewed dysphoric and threat images relative to a mind-wandering control, which was counter to 
the original hypotheses. Overall, attentional negativity bias was not supported as a mechanism by which mindfulness reduces depression.

The finding that trait mindfulness was associated with less attentional negativity bias supports one of the primary predictions. It is important to note that while this pattern was observed in general, not all measures of mindfulness were associated with all measures of gaze patterns. Given that mindfulness is conceptualized in numerous, diverse ways, the fact that not all mindfulness measures were significantly related to gaze patterns is not surprising. Numerous researchers have pointed out that there is widespread disparities in the conceptualization and measurement of mindfulness (e.g. Bergomi, Tschacher, \& Kupper, 2013; Sauer et al., 2013). There has been debate in the field about the factor structure of mindfulness. For example, while the FFMQ originally contained five factors, more recent evidence demonstrates that the FFMQ observe subscale is uncorrelated with other facets of mindfulness (Siegling \& Petrides, 2016). In all, the measurement of mindfulness for research purposes remains relatively new, and difficulties measuring this construct remain.

In general, Study 1 provided evidence that some trait mindfulness measures were related to longer fixation duration and more fixations on positive images and a reduced tendency to first fixate on dysphoric images. Study 2 demonstrated that some trait mindfulness measures were associated with shorter fixation duration and fewer fixations on dysphoric images. FFT was unrelated to trait mindfulness in Study 2. Furthermore, Study 1 found that depression was associated with less time spent viewing positive images, fewer fixations on positive images, and a higher probability of first fixating on a dysphoric image. In contrast, Study 2 found that depression was related to more time spent viewing dysphoric mages and fewer fixations on positive images. Both studies support the hypothesis that depression is related to enhanced 
attentional bias and mindfulness is associated with reduced attentional bias. However, it remains unclear whether these biases occur with positive or negative information.

Other studies on negativity bias in depression have exhibited similar inconsistencies. Some studies show that depression is related to less attention on positive information. For example, Sears and colleagues (2011) found that individuals with elevated depressive symptoms gazed at positive images less than a non-dysphoric group. The paradigm in the present was adapted from Sears and colleagues (2011), and Study 1 replicated this finding. Isaac and colleagues (2014) also replicated this finding using happy faces. Depressed individuals viewed happy faces for a shorter amount of time than a non-depressed control group. In contrast, other studies have found that depression is associated with more attention on negative information. Caseras and colleagues (2007) found that depressive symptoms were associated with increased time spent attending to negative scenes relative to neutral scenes and were unrelated to time spent viewing positive scenes relative to neutral scenes.

There are also similar inconsistencies in studies involving mindfulness and negativity bias. For example, Kiken and Shook (2011) demonstrated that individuals induced with state mindfulness exhibited improved learning of positive information relative to a mind-wandering control group. The two groups though, did not differ in learning of negative information. Meanwhile, other work has demonstrated that mindfulness is associated with a reduction of negatively valenced thoughts (Kiken \& Shook, 2012; 2014).

Taken together, there is support that depression is related to enhanced negativity bias and mindfulness is associated with reduced negativity bias. Though, it is not clear whether the bias is accounted for by differences in positive information or differences in negative information or difference in both. Differences in paradigms and procedures may be able to explain the different 
patterns of findings observed in the literature. Study 1 utilized the same paradigm as Sears and colleagues (2011), but involved different images. The results of both studies were very similar, with depression being related to less time viewing positive images. There are a few differences between Study 1 and Study 2 that could have contributed to the differential findings. It is possible that the attention exercises before the eye tracking procedure may have influenced gaze patterns. Additionally, participants in Study 2 completed the self-report measures in the same room in which they completed the eye-tracking paradigm, and participants in Study 1 completed the self-reports in a different room. Participants who participated in Study 1 were not excluded from Study 2. Some participants may have previously participated in the eye-tracking paradigm and familiarity with the task may have influenced gaze patterns.

Although the correlational evidence in both studies indicate that mindfulness is associated with less attentional negativity bias, there was no support for the proposed mediation model. In Study 1, less attentional negativity bias did not account for the relation between mindfulness and depression. However, there was some evidence for an alternate model whereby mindfulness mediated the relation between attentional negativity bias and depression. That is, less attentional negativity bias was associated with greater trait mindfulness, which in turn, was related to lower levels of depression. Study 2 replicated this finding. There was no evidence that attentional negativity bias mediated the relation between mindfulness and depression. However, mindfulness mediated the relation between attentional negativity bias and depression. Given that this model was counter to the original hypothesis and that it was found in a correlational design, the results should be interpreted with caution. Future research should investigate whether this alternate model can be replicated. However, this model would suggest that changing biased attention in more direct ways may enhance mindfulness. 
There are a few possible explanations that might explain why attentional negativity bias did not mediate the mindfulness-depression association. First, it is possible that the effect size of the mediation model may have been too small to be detected. The present study may have been underpowered. Second, although mindfulness may be related to attentional negativity bias, biased gaze patterns may not be one of the mechanisms by which mindfulness reduces depression. It is unlikely that biased attentional patterns have no effect on mood or well-being. Thus, future research is necessary to determine other possible effects that biased attentional may have.

It was also predicted that a brief state mindfulness induction would cause a decrease in biased attention. The results did not support this hypothesis. Rather, there was some evidence that the mindfulness induction group viewed threat and dysphoric images longer than the mindwandering control group. There was no difference between groups on gaze patterns for any other index of attentional negativity bias. However, this finding suggests that trait mindfulness and state mindfulness have opposite effects on gaze patterns. Whereas trait mindfulness was associated with less attentional bias, a state mindfulness induction caused increased fixation duration on threat and dysphoric images.

That state mindfulness is associated with increased attention on negatively-valenced information is consistent with one conceptualization of mindfulness (Hayes \& Wilson, 2003; Hayes \& Feldman, 2004). In this view, individuals with depression often avoid or over-engage (e.g., ruminate) with negative information, and mindfulness reduces depression by promoting the acceptance of present states without avoidance or over-engagement. In fact, Hayes and Feldman (2004) found that mindfulness was negatively related to experiential avoidance and rumination. Thus, the researchers argued that mindfulness encourages the non-evaluative approach of 
emotional states which in turn reduces emotional distress. This conceptualization has been likened to that of interoceptive exposure (Hayes \& Feldman, 2004). That is, mindfulness encourages the approach of negative thoughts and stimuli which may cause distress in the shortterm, but that exposure may decrease distress in the long-term. In fact, Hayes and colleagues (2007) found that transient worsening of depression symptoms in a mindfulness based psychotherapy treatment for depression was associated with lower levels of depression at posttreatment.

This conceptualization of mindfulness may explain the differential effects of state mindfulness and trait mindfulness observed in Studies 2 and 1, respectively. The brief mindful breathing intervention may have encouraged engagement with negative information, which in turn may have resulted in increased fixation duration on dysphoric and threat images. This finding is consistent with that of Arch and Craske (2006). Participants were instructed to view a series of emotional images presented one at a time as long as they desired. Participants who underwent the same mindful breathing exercise used in the present study viewed negative slides longer than the mind-wandering control group. Therefore, state mindfulness may temporarily increase attention to negative information. However, after repeated exposure to negative information (i.e., being high in trait mindfulness), individuals may be better equipped to face negative information, resulting in decreased attentional bias.

It is important to remember that although a commonly used mindfulness induction and mind-wandering control group was utilized in the present study, the manipulation check did not indicate significant differences in state mindfulness. It is also important to note that the reliability of the state mindfulness measure was below conventional standards for adequate reliability. Therefore, it is impossible to determine whether the manipulation had an effect or not on state 
mindfulness. The finding that the two groups differed on fixation duration on threat and dysphoric images should be interpreted with caution.

There are a few noteworthy limitations to the present studies that have not yet been noted. First, a pattern was observed whereby participants had a tendency to first fixate on the image in the top left corner of the screen, regardless of emotion category. Thus, all findings related to FFT must also be interpreted carefully. Second, although the images were standardized on a number of dimensions that may impact gaze patterns, some differences between image groups still existed. Most notably, there were a higher proportion of images that contained faces in the dysphoric emotion category group than other image types. The greater number of faces in the dysphoric group may have influenced the results observed. Third, there are limitations concerning the mindfulness induction and the mind-wandering induction group. It is possible that a brief, fifteen-minute mindfulness induction may have been too short to cause more significant changes in gaze patterns. Moreover, mind-wandering control groups have been criticized. Some argue that by prompting participants to pay attention to their thoughts as they wander, they are engaging with their present states in a form of mindfulness. In this way, the difference between mind-wandering and mindfulness exercises become clouded. This may also explain why the two groups did not differ in state mindfulness in Study 2. Additionally, both studies involved samples of undergraduates. Both samples contained relatively mild symptoms of depression and some limited variability. Lastly, due to the large number of analyses conducted in each study, type out error rate may have been inflated.

Given these limitations, future research may design paradigms that account for individuals' tendency to first fixate on the top-left corner of a screen. Perhaps FFT should only be included in paradigms that present two images at a time. In particular, an improved measure 
of biases in first fixation may exist in paradigms that only show images at the top and bottom half of a screen. Additionally, the generalization of these findings would be improved if the results were replicated using a different set of images. It would be important for future work also to consider and control for other variables (e.g. contrast, complexity, etc.) that influence gaze patterns as was done in the present study. Furthermore, future research should also incorporate different mindfulness and control group inductions. Specifically, future studies may investigate how a mindfulness intervention aimed to increase dispositional mindfulness, such as mindfulness-based stress reduction, relates to attentional negativity bias. Future research may also using clinical samples with individuals who meet diagnostic criteria for major depression. The current study was an investigation of one potential mechanism of mindfulness. Future work should consider other potential mechanisms such as increased health behaviors or engagement in more valued activities.

Although the results did not support the proposed mediation model, they provided some evidence that trait mindfulness mediates the relation between attentional negativity bias and depression. Furthermore, the results revealed some mixed and inconsistent evidence that trait mindfulness is associated with reduced attentional negativity bias. However, there was some preliminary evidence that state mindfulness may increase attentional negativity. Although mindfulness is largely an attentional process, to the researcher's knowledge, these studies are the first investigations between mindfulness and visual attention. 


\section{References}

Abramson, L. Y., Metalsky, G. I., \& Alloy, L. B. (1989). Hopelessness depression: A theory based subtype of depression. Psychological review, 96, 358.

Alloy, L. B., Abramson, L. Y., Whitehouse, W. G., Hogan, M. E., Panzarella, C., \& Rose, D. T. (2006). Prospective incidence of first onsets and recurrences of depression in individuals at high and low cognitive risk for depression. Journal of abnormal psychology, 115, 145.

American Psychiatric Association. (2013). Diagnostic and statistical manual of mental disorders: DSM-5. Washington, D.C: American Psychiatric Association.

Arch, J. J., \& Craske, M. G. (2006). Mechanisms of mindfulness: Emotion regulation following a focused breathing induction. Behaviour research and therapy, 44, 1849-1858.

Baer, R. A., Smith, G. T., \& Allen, K. B. (2004). Assessment of mindfulness by self-report: The Kentucky Inventory of Mindfulness Skills. Assessment, 11, 191-206.

Baer, R. A., Smith, G. T., Hopkins, J., Krietemeyer, J., \& Toney, L. (2006). Using self-report assessment methods to explore facets of mindfulness. Assessment, 13, 27-45.

Baer, R. A., Smith, G. T., Lykins, E., Button, D., Krietemeyer, J., Sauer, S., ... \& Williams, J. M. G. (2008). Construct validity of the five facet mindfulness questionnaire in meditating and nonmeditating samples. Assessment, 15, 329-342.

Bagby, R. M., Rector, N. A., Bacchiochi, J. R., \& McBride, C. (2004). The stability of the response styles questionnaire rumination scale in a sample of patients with major depression. Cognitive Therapy and Research, 28(4), 527-538.

Barnes, L. L., Harp, D., \& Jung, W. S. (2002). Reliability generalization of scores on the Spielberger state-trait anxiety inventory. Educational and Psychological Measurement, 62(4), 603-618.

Barnhofer, T., Crane, C., Hargus, E., Amarasinghe, M., Winder, R., \& Williams, J. M. G. (2009). Mindfulness-based cognitive therapy as a treatment for chronic depression: a preliminary study. Behaviour Research and Therapy, 47, 366-373.

Baumeister, R.F., Bratslavsky, E., Finkenauer, C., \& Vohs, K.D. (2001). Bad is stronger than good. Review of General Psychology, 5, 323-370.

Beck, A. T. (1987). Cognitive models of depression. Journal of Cognitive Psychotherapy, 1, 537.

Beck, A. T., Epstein, N., Brown, G., \& Steer, R. A. (1988). An inventory of measuring clinical anxiety: Psychometric properties. Journal of Counseling and Clinical Psychology, 56, 893-897. 
Beck, A. T., Steer, R. A., \& Brown, G. K. (1996). Beck Depression Inventory manual (2nd Ed.). San Antonio, TX: Psychological Corporation.

Beevers, C. G., Strong, D. R., Meyer, B., Pilkonis, P. A., \& Miller, I. W. (2007). Efficiently assessing negative cognition in depression: an item response theory analysis of the Dysfunctional Attitude Scale. Psychological assessment, 19(2), 199.

Belsher, G., \& Costello, C. G. (1988). Relapse after recovery from unipolar depression: a critical review. Psychological bulletin, 104, 84.

Bergomi, C., Tschacher, W., \& Kupper, Z. (2013). Measuring mindfulness: first steps towards the development of a comprehensive mindfulness scale. Mindfulness, 4(1), 18-32.

Bieling, P. J., Antony, M. M., \& Swinson, R. P. (1998). The State--Trait Anxiety Inventory, Trait version: structure and content re-examined. Behaviour research and therapy, 36(7), 777 788.

Borkovec, T. D., Ray, W. J., \& Stober, J. (1998). Worry: A cognitive phenomenon intimately linked to affective, physiological, and interpersonal behavioral processes. Cognitive Therapy and Research, 22, 561-576.

Britton, W. B., Haynes, P. L., Fridel, K. W., \& Bootzin, R. R. (2010). Polysomnographic and subjective profiles of sleep continuity before and after mindfulness-based cognitive therapy in partially remitted depression. Psychosomatic Medicine, 72, 539-548.

Broderick, P.C. (2005). Mindfulness and coping with dysphoric mood: Contrasts with rumination and distraction. Cognitive Therapy and Research, 29, 501-510.

Brown, K. W., \& Ryan, R. M. (2003). The benefits of being present: mindfulness and its role in psychological well-being. Journal of personality and social psychology, 84, 822.

Brown, K. W., Ryan, R. M., \& Creswell, J. D. (2007). Mindfulness: Theoretical foundations and evidence for its salutary effects. Psychological Inquiry, 18, 211-237.

Brown, K. W., Weinstein, N., \& Creswell, J. D. (2012). Trait mindfulness modulate neuroendocrine and affective responses to social evaluative threat.

Psychoneuroendocrinology, 37, 2037-2041.

Buchheld, N., Grossman, P., \& Wallach, H. (2001). Measuring mindful- ness in insight meditation (Vipassana) and meditation-based psychotherapy: The development of the Freiburg Mindfulness Inventory (FMI). Journal for Meditation and Meditation Research, $1,11-34$.

Caseras, X., Garner, M., Bradley, B. P., \& Mogg, K. (2007). Biases in visual orienting to negative and positive scenes in dysphoria: An eye movement study. Journal of abnormal psychology, 116(3), 491. 
Chadwick, P., Hember, M., Symes, J., Peters, E., Kuipers, E., \& Dagnan, D. (2008). Responding mindfully to unpleasant thoughts and images: Reliability and validity of the Southampton Mindfulness Questionnaire (SMQ). British Journal of Clinical Psychology, 47, 451-455.

Coffey, K. A., Hartman, M., \& Fredrickson, B. L. (2010). Deconstructing mindfulness and constructing mental health: Understanding mindfulness and its mechanisms of action. Mindfulness, 1(4), 235-253.

Cole, P. M., Michel, M. K., \& Teti, L. O. D. (1994). The development of emotion regulation and dysregulation: A clinical perspective. Monographs of the Society for Research in Child Development, 59, 73-102.

Conklin, L. R., Strunk, D. R., \& Fazio, R. H. (2009). Attitude formation in depression: Evidence for deficits in forming positive attitudes. Journal of behavior therapy and experimental psychiatry, 40(1), 120-126.

Creswell, J. D., \& Lindsay, E. K. (2014). How does mindfulness training affect health? A mindfulness stress buffering account. Current Directions in Psychological Science, 23, 401-407.

Desrosiers, A., Vine, V., Klemanski, D. H., \& Nolen-Hoeksema, S. (2013). Mindfulness and emotion regulation in depression and anxiety: common and distinct mechanisms of action. Depression and anxiety, 30, 654-661.

Faul, F., Erdfelder, E., Buchner, A., \& Lang, A.-G. (2009). Statistical power analyses using G*Power 3.1: tests for correlation and regression analyses. Behavior Research Methods, 41, 1149-60.

Fazio, R. H., Eiser, J. R., \& Shook, N. J. (2004). Attitude formation through exploration: valence asymmetries. Journal of personality and social psychology, 87(3), 293.

Feldman, G., Hayes, A., Kumar, S., Greeson, J., \& Laurenceau, J. (2007). Mindfulness and emotion regulation: The development and initial validation of the Cognitive and Affective Mindfulness Scale-Revised (CAMS-R). Journal of Psychopathology and Behavioral Assessment, 29, 177-190.

Frewen, P. A., Evans, E. M., Maraj, N., Dozois, D. J. A., \& Partridge, K. (2008). Letting go: Mindfulness and negative automatic thinking. Cognitive Therapy and Research, 32, 758774.

Garratt, G., Ingram, R. E., Rand, K. L., \& Sawalani, G. (2007). Cognitive processes in cognitive therapy: Evaluation of the mechanisms of change in the treatment of depression. Clinical Psychology: Science and Practice, 14, 224-239. 
Gilbert, B. D., \& Christopher, M. S. (2010). Mindfulness-based attention as a moderator of the relationship between depressive affect and negative cognitions. Cognitive Therapy and Research, 34, 514-521.

Goeleven, E., De Raedt, R., Baert, S., \& Koster, E. H. (2006). Deficient inhibition of emotional information in depression. Journal of affective disorders, 93(1), 149-157.

Gotlib, I. H. (1984). Depression and general psychopathology in university students. Journal of abnormal psychology, 93(1), 19.

Grossman, P., Niemann, L., Schmidt, S., \& Walach, H. (2004). Mindfulness-based stress reduction and health benefits: A meta-analysis. Journal of psychosomatic research, 57, 35-43.

Hayes, A. M., \& Feldman, G. (2004). Clarifying the construct of mindfulness in the context of emotion regulation and the process of change in therapy. Clinical Psychology: science and practice, 11(3), 255-262.

Hayes, A. M., Laurenceau, J. P., Feldman, G., Strauss, J. L., \& Cardaciotto, L. (2007). Change is not always linear: The study of nonlinear and discontinuous patterns of change in psychotherapy. Clinical psychology review, 27(6), 715-723.

Hayes, A. F., \& Scharkow, M. (2013). The relative trustworthiness of inferential tests of the indirect effect in statistical mediation analysis: Does method really matter? Psychological Science, 24, 1918-1927.

Hayes, S. C., Strosahl, K. D., \& Wilson, K. G. (1999). Acceptance and Commitment Therapy: An experiential approach to behavior change. New York: The Guilford Press.

Hayes, S. C., \& Wilson, K. G. (2003). Mindfulness: Method and process. Clinical Psychology: Science and Practice, 10(2), 161-165.

Herndon, F. (2008). Testing mindfulness with perceptual and cognitive factors: External vs. internal encoding, and the cognitive failures questionnaire. Personality and Individual Differences, 44, 32-41.

Hindash, A. H. C., \& Amir, N. (2012). Negative interpretation bias in individuals with depressive symptoms. Cognitive therapy and research, 36(5), 502-511.

Isaac, L., Vrijsen, J. N., Rinck, M., Speckens, A., \& Becker, E. S. (2014). Shorter gaze duration for happy faces in current but not remitted depression: Evidence from eye movements. Psychiatry research, 218(1), 79-86.

Joormann, J. (2004). Attentional bias in dysphoria: The role of inhibitory processes. Cognition and Emotion, 18(1), 125-147. 
Julian, L. J. (2011), Measures of anxiety: State-Trait Anxiety Inventory (STAI), Beck Anxiety Inventory (BAI), and Hospital Anxiety and Depression Scale-Anxiety (HADS-A). Arthritis Care Research, 63, 467-472.

Just, N., \& Alloy, L. B. (1997). The response styles theory of depression: tests and an extension of the theory. Journal of abnormal psychology, 106, 221.

Kabat-Zinn, J. (1982) An out-patient program in Behavioral Medicine for chronic pain patients based on the practice of mindfulness meditation: Theoretical considerations and preliminary results. General Hospital Psychiatry, 4, 33-47.

Kabat-Zinn, J., Wheeler, E., Light, T., Skillings, A., Scharf, M. J., Cropley, T. G., ... \& Bernhard, J. D. (1998). Influence of a mindfulness meditation-based stress reduction intervention on rates of skin clearing in patients with moderate to severe psoriasis undergoing photo therapy (UVB) and photochemotherapy (PUVA). Psychosomatic medicine, 60, 625-632.

Kabat-Zinn, J. (1990). Full catastrophe living: Using the wisdom of your body and mind to face stress, pain, and illness. New York: Delacourt.

Kabat-Zinn, J. (1994). Wherever You Go, There You Are: Mindfulness Meditation in Everyday Life. New York: Hyperion.

Kabat-Zinn, J., Lipworth, L., \& Burney, R. (1985). The clinical use of mindfulness meditation for the self-regulation of chronic pain. Journal of Behavioral Medicine, 8, 163-190.

Kellough, J. L., Beevers, C. G., Ellis, A. J., \& Wells, T. T. (2008). Time course of selective attention in clinically depressed young adults: An eye tracking study. Behaviour research and therapy, 46(11), 1238-1243.

Khoury, B., Lecomte, T., Fortin, G., Masse, M., Therien, P., Bouchard, V., ... \& Hofmann, S. G. (2013). Mindfulness-based therapy: A comprehensive meta-analysis. Clinical Psychology Review, 33, 763-771.

Kiken, L. G., Garland, E. L., Bluth, K., Palsson, O. S., \& Gaylord, S. A. (2015). From a state to a trait: Trajectories of state mindfulness in meditation during intervention predict changes in trait mindfulness. Personality and Individual Differences.

Kiken, L. G., \& Shook, N. J. (2011). Looking Up Mindfulness Increases Positive Judgments and Reduces Negativity Bias. Social Psychological and Personality Science, 2(4), 425-431.

Kiken, L. G., \& Shook, N. J. (2012). Mindfulness and emotional distress: The role of negatively biased cognition. Personality and Individual Differences, 52(3), 329-333.

Kiken, L. G., \& Shook, N. J. (2014). Does mindfulness attenuate thoughts emphasizing negativity, but not positivity?. Journal of research in personality, 53, 22-30. 
Kingston, T., Dooley, B., Bates, A., Lawlor, E., \& Malone, K. (2007). Mindfulness-based cognitive therapy for residual depressive symptoms. Psychology and Psychotherapy: Theory, Research and Practice, 80, 193-203.

Lang, P. J., Bradley, M. M., \& Cuthbert, B. N. (2005). International affective picture system (IAPS): affective ratings of pictures and instruction manual. Technical Report A-6. Gainesville, FL: University of Florida.

Lenhard, W. \& Lenhard, A. (2014). Hypothesis Tests for Comparing Correlations. available: http://www.psychometrica.de/correlation.html. Bibergau (Germany): Psychometrica.

Linehan, M. M. (1993). Cognitive-behavioral treatment of borderline personality disorder. New York: The Guilford Press.

Ma, S. H., \& Teasdale, J. D. (2004). Mindfulness-based cognitive therapy for depression: replication and exploration of differential relapse prevention effects. Journal of consulting and clinical psychology, 72, 31.

Mathew, K. L., Whitford, H. S., Kenny, M. A., \& Denson, L. A. (2010). The long-term effects of Mindfulness-based Cognitive Therapy as a relapse prevention treatment for major depressive disorder. Behavioural and Cognitive Psychotherapy, 38, 561-576.

Mata, J., Hogan, C. L., Joormann, J., Waugh, C. E., \& Gotlib, I. H. (2013). Acute exercise attenuates negative affect following repeated sad mood inductions in persons who have recovered from depression. Journal of abnormal psychology, 122(1), 45.

Mueller, T. I., Leon, A. C., Keller, M. B., Solomon, D. A., Endicott, J., Coryell, W., ... \& Maser, J. D. (1999). Recurrence after recovery from major depressive disorder during 15 years of observational follow-up. American Journal of Psychiatry, 156, 1000-1006.

Nolen-Hoeksema, S. (2000). The role of rumination in depressive disorders and mixed anxiety/depressive symptoms. Journal of abnormal psychology, 109(3), 504.

Nolen-Hoeksema, S., Wisco, B. E., \& Lyubomirsky, S. (2008). Rethinking rumination. Perspectives on Psychological Science, 3, 400-424.

Pergamin-Hight, L., Naim, R., Bakermans-Kranenburg, M. J., van IJzendoorn, M. H., \& BarHaim, Y. (2015). Content specificity of attention bias to threat in anxiety disorders: A metaanalysis. Clinical Psychology Review, 3510-3518.

Piet, J., \& Hougaard, E. (2011). The effect of mindfulness-based cognitive therapy for prevention of relapse in recurrent major depressive disorder: a systematic review and meta-analysis. Clinical psychology review, 31(6), 1032-1040. 
Preacher, K. J., \& Hayes, A. F. (2004). SPSS and SAS procedures for estimating indirect effects in simple mediation models. Behavior Research Methods, Instruments, and Computers, 36, 717-731.

Preacher, K. J., \& Hayes, A. F. (2008). Asymptotic and resampling strategies for assessing and comparing indirect effects in multiple mediator models. Behavior Research Methods, 40, 879-891.

Raes, F., \& Williams, J. M. G. (2010). The relationship between mindfulness and uncontrollability of ruminative thinking. Mindfulness, 1, 199-203.

Regier, D. A., Rae, D. S., Narrow, W. E., Kaelber, C. T., \& Schatzberg, A. F. (1998). Prevalence of anxiety disorders and their comorbidity with mood and addictive disorders. The British Journal Of Psychiatry, 173, 24-28

Roberts, J. E., Gilboa, E., \& Gotlib, I. H. (1998). Ruminative response style and vulnerability to episodes of dysphoria: Gender, neuroticism, and episode duration. Cognitive Therapy and Research, 22(4), 401-423.

Roelofs, J., Muris, P., Huibers, M., Peeters, F., \& Arntz, A. (2006). On the measurement of rumination: A psychometric evaluation of the ruminative response scale and the rumination on sadness scale in undergraduates.Journal of behavior therapy and experimental psychiatry, 37(4), 299-313.

Rozin, P., \& Royzman, E. B. (2001). Negativity bias, negativity dominance, and contagion. Personality and Social Psychology Review, 5, 296-320.

Sauer, S., Walach, H., Schmidt, S., Hinterberger, T., Lynch, S., Büssing, A., \& Kohls, N. (2013). Assessment of mindfulness: Review on state of the art. Mindfulness, 4(1), 3-17.

Sears, C. R., Newman, K. R., Ference, J. D., \& Thomas, C. L. (2011). Attention to emotional images in previously depressed individuals: An eye-tracking study. Cognitive therapy and research, 35(6), 517-528.

Segal, Z. V., Williams, J. M. G., \& Teasdale, J. D. (2002). Mindfulness-based cognitive therapy for depression: A new approach to preventing relapse. New York: The Guilford Press.

Shook, N. J., Fazio, R. H., \& Vasey, M. W. (2007). Negativity bias in attitude learning: A possible indicator of vulnerability to emotional disorders?. Journal of behavior therapy and experimental psychiatry, 38(2), 144-155.

Siegling, A. B., \& Petrides, K. V. (2016). Zeroing In on Mindfulness Facets: Similarities, Validity, and Dimensionality across Three Independent Measures. PloS one, 11(4), e0153073. 
Spielberger, C. D., Gorsuch, R. L., Lushene, R., Vagg, P. R., \& Jacobs, G. A. (1983). Manual for the State-Trait Anxiety Inventory. Palo Alto, CA: Consulting Psychologists Press

Teasdale, J. D., Segal, Z. V., Williams, J. M. G., Ridgeway, V. A., Soulsby, J. M. and Lau, M. A. (2000). Prevention of relapse/recurrence in major depression by mindfulness-based cognitive therapy. Journal of Consulting and Clinical Psychology, 68, 615-623.

Thompson, R. A. (1994). Emotion regulation: A theme in search of definition. Monographs of the society for research in child development, 59, 25-52.

Treynor, Gonzalez, \& Nolen-Hoeksema (2003). Rumination Reconsidered: A Psychometric Analysis Cognitive Therapy and Research, 27, 247-259.

Ussher, M., Spatz, A., Copland, C., Nicolaou, A., Cargill, A., Amini-Tabrizi, N., \& McCracken, L. M. (2014). Immediate effects of a brief mindfulness-based body scan on patients with chronic pain. Journal Of Behavioral Medicine, 37(1), 127-134.

Watson, D., Clark, L. a, \& Tellegen, a. (1988). Development and validation of brief measures of positive and negative affect: the PANAS scales. Journal of Personality and Social Psychology, 54, 1063-1070.

Weissman, A. N., \& Beck, A. T. (1978). Development and validation of the Dysfunctional Attitude Scale. Chicago: Paper presented at the annual meeting of the Association for the Advancement of Behavior Therapy.

Wenk-Sormaz, H. (2005). Meditation can reduce habitual responding. Advances in Mind-Body Medicine, 21(3-4), 33-49. 
Table 1. Pilot Study Descriptive Statistics for Image Categories

\begin{tabular}{|c|c|c|c|c|c|}
\hline Index & Positive & Dysphoric & Threat & Neutral & $\begin{array}{c}\text { Group } \\
\text { Comparisons }\end{array}$ \\
\hline Happy & $3.02(0.60)^{\mathrm{a}}$ & $1.06(0.08)^{b}$ & $1.07(0.06)^{\mathrm{b}}$ & $1.27(0.25)^{\mathrm{b}}$ & $\begin{array}{c}F(3,31)=67.55 \\
p<.001\end{array}$ \\
\hline Dysphoric & $1.10(0.07)^{\mathrm{a}}$ & $3.52(0.50)^{b}$ & $1.65(0.34)^{\mathrm{c}}$ & $1.14(0.11)^{\mathrm{a}}$ & $\begin{array}{c}F(3,31)=107.89 \\
p<.001\end{array}$ \\
\hline Scared & $1.67(0.84)^{\mathrm{a}}$ & $1.90(0.50)^{\mathrm{a}}$ & $3.36(0.29)^{b}$ & $1.07(0.08)^{\mathrm{c}}$ & $\begin{array}{c}F(3,31)=28.57 \\
p<.001\end{array}$ \\
\hline Luminescence & $105.31(23.43)^{\mathrm{a}}$ & $105.07(25.79)^{\mathrm{a}}$ & $79.89(19.72)^{\mathrm{a}}$ & $103.27(30.31)^{\mathrm{a}}$ & $\begin{array}{c}F(3,31)=1.94 \\
p=.15\end{array}$ \\
\hline $\begin{array}{l}\text { Color Saturation - } \\
\text { Red }\end{array}$ & $110.96(27.41)^{\mathrm{a}}$ & $115.25(27.47)^{\mathrm{a}}$ & $82.94(30.48)^{a}$ & $113.66(24.30)^{\mathrm{a}}$ & $\begin{array}{c}F(3,31)=2.47 \\
p>.08\end{array}$ \\
\hline $\begin{array}{l}\text { Color Saturation - } \\
\text { Green }\end{array}$ & $105.28(22.96)^{\mathrm{a}}$ & $102.22(24.91)^{\mathrm{a}}$ & $79.60(21.74)^{\mathrm{a}}$ & $101.20(33.84)^{\mathrm{a}}$ & $\begin{array}{c}F(3,31)=1.61 \\
p=.21\end{array}$ \\
\hline $\begin{array}{l}\text { Color Saturation - } \\
\text { Blue }\end{array}$ & $90.40(27.95)^{\mathrm{a}}$ & $92.89(29.34)^{\mathrm{a}}$ & $73.33(27.34)^{\mathrm{a}}$ & $86.35(38.75)^{a}$ & $\begin{array}{c}F(3,31)=0.62 \\
p=.61\end{array}$ \\
\hline Contrast & $0.57(0.11)^{\mathrm{a}}$ & $0.61(0.18)^{\mathrm{a}}$ & $0.75(0.21)^{\mathrm{a}}$ & $0.57(0.18)^{\mathrm{a}}$ & $\begin{array}{c}F(3,31)=1.97 \\
p=.14\end{array}$ \\
\hline Complexity & $\begin{array}{c}85,830.50 \\
(30,749.14)^{\mathrm{a}}\end{array}$ & $\begin{array}{c}45,385.75 \\
(20,518.77)^{\mathrm{a}}\end{array}$ & $\begin{array}{c}63,452.50 \\
(30,085.83)^{\mathrm{a}}\end{array}$ & $\begin{array}{c}65,850.25 \\
(28,780.12)^{\mathrm{a}}\end{array}$ & $\begin{array}{c}F(3,31)=2.83 \\
p=.06\end{array}$ \\
\hline Valence & $6.87(0.45)^{\mathrm{a}}$ & $2.73(0.52)^{b}$ & $2.62(0.48)^{b}$ & $5.04(0.32)^{\mathrm{c}}$ & $\begin{array}{c}F(3,31)=203.82 \\
p<.001\end{array}$ \\
\hline Arousal & $4.55(1.75)^{\mathrm{a}}$ & $4.12(0.64)^{\mathrm{a}}$ & $5.34(0.70)^{\mathrm{a}}$ & $1.51(0.18)^{\mathrm{b}}$ & $\begin{array}{c}F(3,31)=26.15 \\
p<.001\end{array}$ \\
\hline
\end{tabular}


Table 2. Study 1 Descriptive Statistics for Eye Tracking Indices

\begin{tabular}{clc}
\hline Eye Tracking Index & Image Category & Mean (SD) \\
\hline FD Ratio & Positive & $.24(0.05)$ \\
& Dysphoric & $.28(0.07)$ \\
& Threat & $.21(0.05)$ \\
& Neutral & $.14(0.04)$ \\
& Black area & $.12(0.07)$ \\
FC Ratio & & \\
& Positive & $.23(.05)$ \\
& Dysphoric & $.29(.06)$ \\
& Threat & $.20(.04)$ \\
& Neutral & $.15(.03)$ \\
Black area & $.14(.07)$ \\
FFT & Positive & \\
& Dysphoric & $5.12(1.54)$ \\
& Threat & $7.06(1.80)$ \\
& Neutral & $5.47(1.70)$ \\
& &
\end{tabular}

FD Difference Scores

$$
\begin{array}{ll}
\text { Dysphoric - Positive } & .05(.10) \\
\text { Dysphoric - Threat } & .07(.09) \\
\text { Dysphoric - Neutral } & .15(.09)
\end{array}
$$

FC Difference Scores

$$
\text { Dysphoric - Positive } \quad .06(.08)
$$




$$
\begin{array}{ll}
\text { Dysphoric - Threat } & .09(.07) \\
\text { Dysphoric - Neutral } & .14(.07)
\end{array}
$$

Note. FD = Fixation Duration; FC = Fixation Count; FFT = First Fixation Type. 
Table 3. Study 1 Descriptive Statistics for Self-Report Measures

\begin{tabular}{lccc}
\hline Measure & Mean (SD) & Range & Cronbach’s Alpha \\
\hline BDI -II & $10.62(7.92)$ & $0.00-43.00$ & .88 \\
RRS - R & $9.66(3.15)$ & $5.00-18.00$ & .80 \\
RRS - B & $8.14(2.91)$ & $5.00-17.00$ & .78 \\
DAS- SF1 & $19.60(4.66)$ & $10.00-32.00$ & .81 \\
STAI - Trait & $42.54(10.66)$ & $21.00-68.00$ & .91 \\
BAI & $12.43(9.51)$ & $0.00-40.00$ & .91 \\
PANAS - PA & $27.73(6.98)$ & $12.00-44.00$ & .86 \\
PANAS - NA & $13.50(4.75)$ & $10.00-34.00$ & .86 \\
MAAS & $3.79(0.86)$ & $2.00-5.00$ & .89 \\
CAMS-R & $2.52(0.53)$ & $1.20-3.80$ & .81 \\
FFMQ - observing & $25.48(4.89)$ & $40.00-66.00$ & .76 \\
FFMQ - describing & $25.02(6.52)$ & $8.00-39.00$ & .90 \\
FFMQ - awareness & $25.71(6.22)$ & $8.00-35.00$ & .91 \\
FFMQ - non- judgment & $26.64(6.45)$ & $11.00-40.00$ & .84 \\
FFMQ - non-reactance & $20.14(4.25)$ & $11.00-32.00$ & .77
\end{tabular}

Note. PANAS- PA= Positive and Negative Affect Schedule - Positive Affect; PANAS - NA= Positive and Negative Affect Schedule - Negative Affect; BDI-II = Beck Depression InventoryII; DAS-SF1 = Dysfunctional Attitudes Scale-Short Form-1; RRS-R = Ruminative Response Scale-Reflection; RRS-B = Ruminative Response Scale-Brooding; BAI = Beck Anxiety Inventory; STAI-State $=$ State-Trait Anxiety Inventory - Trait; CAMSR $=$ Cognitive and Affective Mindfulness Scale Revised; MAAS - Mindful Attention Awareness Scale; FFMQ = Five Facet Mindfulness Questionnaire. 
Table 4. Study 1 Self-report correlations

\begin{tabular}{|c|c|c|c|c|c|c|c|c|c|c|c|c|c|c|c|}
\hline & \multicolumn{15}{|c|}{ Correlation Coefficients } \\
\hline & 1 & 2 & 3 & 4 & 5 & 6 & 7 & 8 & 9 & 10 & 11 & 12 & 13 & 14 & 15 \\
\hline 1. PANAS-PA & 1 & & & & & & & & & & & & & & \\
\hline 2. PANAS-NA & $.16^{\dagger}$ & 1 & & & & & & & & & & & & & \\
\hline 3. BDI & $-.20^{*}$ & $.41^{* *}$ & 1 & & & & & & & & & & & & \\
\hline 4. DAS-SF1 & $-.18^{\dagger}$ & $.22^{*}$ & $.41^{* *}$ & 1 & & & & & & & & & & & \\
\hline 5. RRS-R & -.10 & $.31^{* *}$ & $.69^{* *}$ & $.27^{* *}$ & 1 & & & & & & & & & & \\
\hline 6. RRS-B & -.01 & $.24^{* *}$ & $.36^{* *}$ & $.17^{\dagger}$ & $.44^{* *}$ & 1 & & & & & & & & & \\
\hline 7. BAI & .05 & $.48^{* *}$ & $.43^{* *}$ & $.17^{\dagger}$ & $.49^{* *}$ & $.22^{*}$ & 1 & & & & & & & & \\
\hline 8. STAI-T & $-.32^{* *}$ & $.36^{* *}$ & $.72^{* *}$ & $.49^{* *}$ & $.68^{* *}$ & $.33^{* *}$ & $.41^{* *}$ & 1 & & & & & & & \\
\hline 9. CAMSR & $.34^{* *}$ & -.11 & $-.41^{* *}$ & $-.22^{*}$ & $-.32^{* *}$ & -.12 & $-.17^{\dagger}$ & $-.59^{* *}$ & 1 & & & & & & \\
\hline 10. MAAS & $.21^{*}$ & $-.19^{*}$ & $-.43^{* *}$ & $-.38^{* *}$ & $-.42^{* *}$ & $-.30^{* *}$ & $-.27^{* *}$ & $-.50^{* *}$ & $.43^{* *}$ & 1 & & & & & \\
\hline 11. FFMQ-observing & $.31^{* *}$ & .03 & -.14 & $-.17^{\dagger}$ & -.12 & .07 & $.17^{\dagger}$ & $-.19^{*}$ & $.38^{* *}$ & .11 & 1 & & & & \\
\hline 12. FFMQ-nonjudging & .08 & $-.18^{*}$ & $-.35^{* *}$ & $-.21^{*}$ & $-.52^{* *}$ & $-.41^{* *}$ & $-.19^{*}$ & $-.59^{* *}$ & $.39^{* *}$ & $.45^{* *}$ & -.07 & 1 & & & \\
\hline 13. FFMQ-describing & $.27^{* *}$ & $-.19^{*}$ & $-.35^{* *}$ & $-.21^{*}$ & $-.32^{* *}$ & -.00 & $-.23^{*}$ & $-.47^{* *}$ & $.58^{* *}$ & $.31^{* *}$ & $.39^{* *}$ & $.18^{*}$ & 1 & & \\
\hline 14. FFMQ-awareness & $.17^{\dagger}$ & $-.18^{*}$ & $-.48^{* *}$ & $-.27^{* *}$ & $-.40^{* *}$ & $-.23^{*}$ & $-.22^{*}$ & $-.49^{* *}$ & $.56^{* *}$ & $.79^{* *}$ & .09 & $.44^{* *}$ & $.35^{* *}$ & 1 & \\
\hline 15. FFMQ-nonreactance & .13 & $-.22^{*}$ & $-.19^{*}$ & $-.16^{\dagger}$ & $-.23^{*}$ & .03 & $-.17^{\dagger}$ & $-.26^{* *}$ & $.41^{* *}$ & $.21^{*}$ & $.35^{* *}$ & .06 & $.33^{* *}$ & $.16^{\dagger}$ & 1 \\
\hline
\end{tabular}

Note. ${ }^{* *} p<0.01 ; * p<0.05 ; \dagger p<0.10$. PANAS- PA= Positive and Negative Affect Schedule - Positive Affect; PANAS - NA= Positive and Negative Affect Schedule - Negative Affect; BDI-II = Beck Depression Inventory-II; DAS-SF1 = Dysfunctional Attitudes Scale-Short Form-1; RRS-R = Ruminative Response Scale-Reflection; RRS-B = Ruminative Response Scale-Brooding; BAI = Beck Anxiety Inventory; STAI-State = State-Trait Anxiety Inventory - Trait; CAMSR = Cognitive and Affective Mindfulness Scale Revised; MAAS - Mindful Attention Awareness Scale; FFMQ = Five Facet Mindfulness Questionnaire. 
Table 5. Study 1 FD Ratio and Difference Score Correlations

\begin{tabular}{|c|c|c|c|c|c|c|c|c|}
\hline & \multicolumn{8}{|c|}{ Correlation Coefficients } \\
\hline & $\begin{array}{c}\text { FD } \\
\text { Ratio- } \\
\text { Positive }\end{array}$ & $\begin{array}{l}\text { FD Ratio- } \\
\text { Dysphoric }\end{array}$ & $\begin{array}{c}\text { FD } \\
\text { Ratio- } \\
\text { Threat }\end{array}$ & $\begin{array}{c}\text { FD } \\
\text { Ratio- } \\
\text { Neutral }\end{array}$ & $\begin{array}{c}\text { FD } \\
\text { Ratio- } \\
\text { Black }\end{array}$ & $\begin{array}{l}\text { Dysphoric } \\
\text { minus } \\
\text { Positive }\end{array}$ & $\begin{array}{c}\text { Dysphoric } \\
\text { minus } \\
\text { Threat }\end{array}$ & $\begin{array}{c}\text { Dysphoric } \\
\text { minus } \\
\text { Neutral }\end{array}$ \\
\hline PANAS_PA & -.09 & -.01 & .09 & -.10 & .06 & .04 & -.05 & .04 \\
\hline PANAS_NA & -.17 & $.21^{*}$ & .02 & .02 & -.09 & $.24^{* *}$ & .15 & $.15^{\dagger}$ \\
\hline BDI & $-.20^{*}$ & .16 & -.03 & -.05 & .05 & $.21^{*}$ & .12 & .13 \\
\hline DAS_SF & -.14 & .02 & .04 & .02 & .05 & .08 & -.02 & .00 \\
\hline RRS-R & $-.17^{\dagger}$ & .12 & -.07 & .06 & .02 & $.18^{*}$ & .13 & .07 \\
\hline RRS-B & -.06 & .13 & -.03 & .09 & -.11 & .12 & .11 & .06 \\
\hline BAI & -.08 & .11 & -.12 & .10 & -.02 & .12 & .15 & .04 \\
\hline STAI - T & -.14 & .08 & -.11 & .06 & -.07 & .13 & .12 & .03 \\
\hline CAMS-R & $.18^{*}$ & -.10 & $-.23^{*}$ & -.02 & $-.18 *$ & $-.17^{\dagger}$ & $-.20^{*}$ & -.06 \\
\hline MAAS & .01 & -.07 & -.11 & .06 & -.04 & -.06 & -.11 & -.08 \\
\hline $\begin{array}{l}\text { FFMQ- } \\
\text { observing }\end{array}$ & -.05 & $.17^{\dagger}$ & .04 & -.12 & -.10 & $.15^{+}$ & .11 & $.18^{*}$ \\
\hline $\begin{array}{l}\text { FFMQ- } \\
\text { nonjudging }\end{array}$ & .14 & -.13 & .06 & -.02 & .00 & $-.17^{\dagger}$ & -.13 & -.10 \\
\hline $\begin{array}{l}\text { FFMQ- } \\
\quad \text { describing }\end{array}$ & .04 & .10 & .09 & -.07 & $-.15^{\dagger}$ & .05 & .03 & .11 \\
\hline $\begin{array}{l}\text { FFMQ- } \\
\quad \text { awareness }\end{array}$ & $.20 *$ & -.01 & .05 & .04 & $-.18^{*}$ & -.11 & -.03 & -.02 \\
\hline $\begin{array}{l}\text { FFMQ- } \\
\text { nonreactance }\end{array}$ & $.17^{\dagger}$ & .02 & .14 & -.10 & $-.18 *$ & -.08 & -.06 & .05 \\
\hline
\end{tabular}

Note. ${ }^{* *} p<0.01 ; * p<0.05 ; \dagger p<0.10$. PANAS- PA= Positive and Negative Affect Schedule - Positive Affect; PANAS - NA= Positive and Negative Affect Schedule - Negative Affect; BDI-II = Beck Depression Inventory-II; DAS-SF1 = Dysfunctional Attitudes Scale-Short Form-1; RRS-R = Ruminative Response Scale-Reflection; RRS-B = Ruminative Response Scale-Brooding; BAI = Beck Anxiety Inventory; STAI-State = State-Trait Anxiety Inventory - Trait; CAMSR = Cognitive and Affective Mindfulness Scale Revised; MAAS - Mindful Attention Awareness Scale; FFMQ = Five Facet Mindfulness Questionnaire; FD = Fixation Duration. 
Table 6. Study 1 FC Ratio and Difference Score Correlations

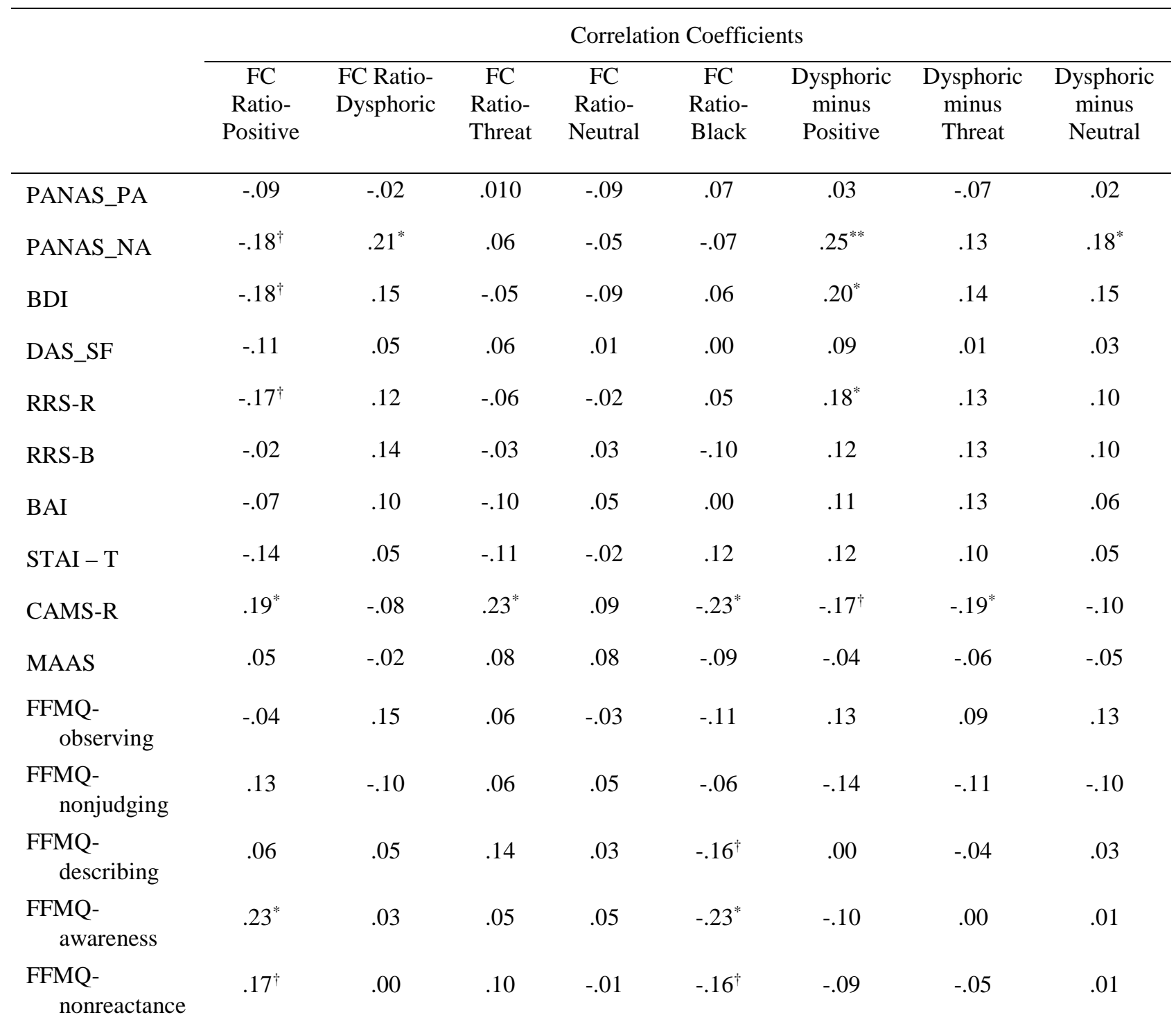

Note. ${ }^{* *} p<0.01 ; * p<0.05 ; \dagger p<0.10$. PANAS- PA= Positive and Negative Affect Schedule - Positive Affect; PANAS - NA= Positive and Negative Affect Schedule - Negative Affect; BDI-II = Beck Depression Inventory-II; DAS-SF1 = Dysfunctional Attitudes Scale-Short Form-1; RRS-R = Ruminative Response Scale-Reflection; RRS-B = Ruminative Response Scale-Brooding; BAI = Beck Anxiety Inventory; STAI-State = State-Trait Anxiety Inventory - Trait; CAMSR = Cognitive and Affective Mindfulness Scale Revised; MAAS - Mindful Attention Awareness Scale; FFMQ = Five Facet Mindfulness Questionnaire; FC = Fixation Count. 
Table 7. Study 1 FFT Correlations

\begin{tabular}{|c|c|c|c|c|}
\hline & \multicolumn{4}{|c|}{ Correlation Coefficients } \\
\hline & $\begin{array}{l}\text { FFT - } \\
\text { Positive }\end{array}$ & FFT - Dysphoric & $\begin{array}{l}\text { FFT- } \\
\text { Threat }\end{array}$ & $\begin{array}{l}\text { FFT - } \\
\text { Neutral }\end{array}$ \\
\hline PANAS_PA & -.03 & -.07 & .11 & .00 \\
\hline PANAS_NA & -.13 & .16 & -.07 & .01 \\
\hline BDI & -.09 & $.18^{*}$ & -.14 & .02 \\
\hline DAS_SF & .14 & -.02 & -.17 & .02 \\
\hline RRS-R & .02 & $.22^{*}$ & -.15 & -.09 \\
\hline RRS-B & -.02 & .09 & .00 & -.06 \\
\hline BAI & -.05 & .13 & .02 & -.11 \\
\hline $\mathrm{STAI}-\mathrm{T}$ & -.09 & $.27^{* *}$ & $-.19^{*}$ & -.04 \\
\hline CAMS-R & .10 & $-.21^{*}$ & .05 & .08 \\
\hline MAAS & .13 & $-.15^{\dagger}$ & .01 & -.01 \\
\hline FFMQ-observing & .01 & .00 & .03 & -.03 \\
\hline FFMQ-nonjudging & .08 & $-.17^{\dagger}$ & .06 & .06 \\
\hline FFMQ-describing & -.02 & -.08 & .03 & .06 \\
\hline FFMQ-awareness & $.19^{*}$ & $-.17^{\dagger}$ & .04 & -.06 \\
\hline FFMQ-nonreactance & -.02 & -.03 & -.04 & .09 \\
\hline
\end{tabular}

Note: ${ }^{* *} p<0.01 ; * p<0.05 ; \dagger p<0.10$. PANAS- PA= Positive and Negative Affect Schedule - Positive Affect; PANAS - NA= Positive and Negative Affect Schedule - Negative Affect; BDI-II = Beck Depression Inventory-II; DAS-SF1 = Dysfunctional Attitudes Scale-Short Form-1; RRS-R = Ruminative Response Scale-Reflection; RRS-B $=$ Ruminative Response Scale-Brooding; BAI = Beck Anxiety Inventory; STAI-State = State-Trait Anxiety Inventory - Trait; CAMSR = Cognitive and Affective Mindfulness Scale Revised; MAAS - Mindful Attention Awareness Scale; FFMQ = Five Facet Mindfulness Questionnaire; FFT = First Fixation Type. 
Table 8. Study 2 Descriptive Statistics for Eye Tracking Indices

\begin{tabular}{llll}
\hline & & Total Sample & Mindfulness \\
Eye Tracking Index & Image Category & Mean (SD) & $\begin{array}{l}\text { Mind-wandering } \\
\text { Mean (SD) }\end{array}$ \\
\hline
\end{tabular}

FD Ratio

$\begin{array}{llll}\text { Positive } & .25(.06) & .24(.06) & .26(.07) \\ \text { Dysphoric } & .28(.06) & .29(.06) & .27(.05) \\ \text { Threat } & .21(.04) & .22(.05) & .20(.04) \\ \text { Neutral } & .15(.04) & .14(.04) & .15(.04) \\ \text { Black area } & .10(.04) & .10(.07) & .11(.09)\end{array}$

FC Ratio

$\begin{array}{llll}\text { Positive } & .24(.05) & .24(.04) & .25(.05) \\ \text { Dysphoric } & .28(.05) & .28(.05) & .27(.05) \\ \text { Threat } & .20(.04) & .21(.04) & .18(.03) \\ \text { Neutral } & .15(.03) & .15(.03) & .15(.03) \\ \text { Black area } & .13(.06) & .12(.06) & .13(.06)\end{array}$

FFT

$\begin{array}{llll}\text { Positive } & 4.22(1.68) & 4.26(1.61) & 4.30(1.65) \\ \text { Dysphoric } & 5.53(1.99) & 5.56(2.01) & 5.58(1.89) \\ \text { Threat } & 4.62(1.72) & 4.48(1.58) & 4.83(1.77) \\ \text { Neutral } & 4.65(1.76) & 4.84(1.60) & 4.51(1.84)\end{array}$

FD Difference Scores

$\begin{array}{llll}\text { Dysphoric - Positive } & .03(.09) & .04(.09) & .01(.09) \\ \text { Dysphoric - Threat } & .07(.07) & .07(.08) & .07(.06) \\ \text { Dysphoric - Neutral } & .14(.08) & .15(.08) & .12(.08)\end{array}$

FC Difference Scores

$\begin{array}{llll}\text { Dysphoric - Positive } & .03(.07) & .04(.07) & .02(.08) \\ \text { Dysphoric - Threat } & .07(.06) & .07(.06) & .08(.06)\end{array}$


Note. $\mathrm{FD}=$ Fixation Duration; FC = Fixation Count; FFT = First Fixation Type. 
Table 9. Study 2 Descriptive Statistics for Self-Report Measures

\begin{tabular}{|c|c|c|c|c|}
\hline Measure & $\begin{array}{c}\text { Total Sample } \\
\text { Mean (SD) }\end{array}$ & $\begin{array}{l}\text { Mindfulness } \\
\text { Mean (SD) }\end{array}$ & $\begin{array}{c}\text { Mind-wandering } \\
\text { Mean (SD) }\end{array}$ & $\begin{array}{c}\text { Total Sample } \\
\text { Cronbach's Alpha }\end{array}$ \\
\hline BDI -II & $12.89(7.21)$ & $12.50(6.83)$ & $13.35(7.66)$ & .84 \\
\hline RRS - R & $10.44(3.16)$ & $10.18(3.30)$ & $10.73(3.02)$ & .76 \\
\hline RRS - B & $8.46(3.11)$ & $8.28(3.18)$ & $8.65(3.07)$ & .78 \\
\hline DAS- SF1 & $18.98(3.87)$ & $19.08(4.21)$ & $18.83(3.55)$ & .71 \\
\hline STAI - State & 39.17 (10.23) & $39.22(10.74)$ & $39.30(9.76)$ & .74 \\
\hline STAI -Trait & $43.37(9.97)$ & $42.98(10.02)$ & $43.98(9.92)$ & .90 \\
\hline BAI & $13.19(8.68)$ & $13.25(8.31)$ & $13.06(9.15)$ & .88 \\
\hline PANAS - PA & $25.67(6.67)$ & $25.92(6.47)$ & $25.43(8.64)$ & .88 \\
\hline PANAS - NA & $14.99(4.76)$ & $15.10(5.14)$ & $14.92(4.42)$ & .78 \\
\hline Mood - Negative & $2.48(1.38)$ & $2.39(1.49)$ & $2.57(1.28)$ & $\mathrm{n} / \mathrm{a}$ \\
\hline Mood - Positive & $4.83(1.21)$ & $4.89(1.27)$ & $4.77(1.56)$ & $\mathrm{n} / \mathrm{a}$ \\
\hline State MAAS & $17.17(5.33)$ & $17.28(5.60)$ & $17.07(4.98)$ & .63 \\
\hline MAAS & $3.64(0.74)$ & $3.60(.63)$ & $3.70(.83)$ & .84 \\
\hline CAMS-R & $2.51(0.50)$ & $2.55(.46)$ & $2.49(.54)$ & .71 \\
\hline FFMQ - observing & $25.42(5.42)$ & $25.37(5.38)$ & $25.63(5.42)$ & .76 \\
\hline FFMQ - describing & $25.11(6.06)$ & $25.43(6.45)$ & $24.78(5.74)$ & .89 \\
\hline FFMQ - awareness & $24.87(5.40)$ & $24.55(5.10)$ & $5.28(5.71)$ & .87 \\
\hline FFMQ - non-judgment & $22.88(5.20)$ & $22.97(4.72)$ & $22.82(5.73)$ & .86 \\
\hline FFMQ - non-reactance & $19.79(4.21)$ & $20.40(3.92)$ & $19.25(4.46)$ & .77 \\
\hline
\end{tabular}


Note. BDI-II = Beck Depression Inventory-II; RRS-R = Ruminative Response Scale-Reflection; RRS-B = Ruminative Response Scale-Reflection; DAS-SF1 = Dysfunctional Attitudes Scale-Short Form 1; STAI-State = State-Trait Anxiety Inventory - State; BAI = Beck Anxiety Inventory; PANAS- PA= Positive and Negative Affect Schedule - Positive Affect; PANAS - NA= Positive and Negative Affect Schedule - Negative Affect; Mood -

Negative $=$ Mood Questionnaire - negative mood; Mood - Positive $=$ Mood Questionnaire - positive mood; MAAS - Mindful Attention Awareness Scale; FFMQ = Five Facet Mindfulness Questionnaire. 
Table 10. Study 2 Self-report correlations

\begin{tabular}{|c|c|c|c|c|c|c|c|c|c|c|c|c|c|c|c|c|}
\hline & \multicolumn{16}{|c|}{ Correlation Coefficients } \\
\hline & 1 & 2 & 3 & 4 & 5 & 6 & 7 & 8 & 9 & 10 & 11 & 12 & 13 & 14 & 15 & 16 \\
\hline 1. PANAS-PA & 1 & & & & & & & & & & & & & & & \\
\hline 2. PANAS-NA & .09 & 1 & & & & & & & & & & & & & & \\
\hline 3. BDI & -.13 & $.29^{* *}$ & 1 & & & & & & & & & & & & & \\
\hline 4. DAS-SF1 & .01 & $.26^{* *}$ & $.44^{* *}$ & 1 & & & & & & & & & & & & \\
\hline 5. RRS-R & -.01 & $.27^{* *}$ & $.47^{* *}$ & $.32^{* *}$ & 1 & & & & & & & & & & & \\
\hline 6. RRS-B & .10 & $.20^{*}$ & $.37^{* *}$ & $.17^{\dagger}$ & $.56^{* *}$ & 1 & & & & & & & & & & \\
\hline 7. BAI & -.05 & $.29^{* *}$ & $.51^{* *}$ & $.27^{* *}$ & $.45^{* *}$ & $.22^{*}$ & 1 & & & & & & & & & \\
\hline 8. STAI-S & $-.18^{*}$ & $.51^{* *}$ & $.62^{* *}$ & $.46^{* *}$ & $.41^{* *}$ & $.25^{* *}$ & $.41^{* *}$ & 1 & & & & & & & & \\
\hline 9. STAI-T & $-.18^{\dagger}$ & $.25^{* *}$ & $.77^{* *}$ & $.43^{* *}$ & $.57^{* *}$ & $.33^{* *}$ & $.51^{* *}$ & $.74^{* *}$ & 1 & & & & & & & \\
\hline 10. CAMSR & $.36^{* *}$ & $-.17^{\dagger}$ & $-.55^{* *}$ & $-.34^{* *}$ & $-.32^{* *}$ & -.11 & $-.39^{* *}$ & $-.52^{* *}$ & $-.65^{* *}$ & 1 & & & & & & \\
\hline 11. MAAS & .13 & $-.22^{*}$ & $-.52^{* *}$ & $-.34^{* *}$ & $-.38^{* *}$ & $-.29^{* *}$ & $-.39^{* *}$ & $-.33^{* *}$ & $-.42^{* *}$ & $.50^{* *}$ & 1 & & & & & \\
\hline 12. FFMQ-observing & $.27^{* *}$ & .01 & -.12 & -.09 & .02 & .05 & .14 & -.14 & -.10 & $.33^{* *}$ & .13 & 1 & & & & \\
\hline 13. FFMQ-nonjudging & .02 & $-.17^{\dagger}$ & $-.43^{* *}$ & $-.26^{* *}$ & $-.41^{* *}$ & $-.26^{* *}$ & $-.28^{* *}$ & $-.28^{* *}$ & $-.49^{* *}$ & $.30^{* *}$ & $.35^{* *}$ & -.09 & 1 & & & \\
\hline 14. FFMQ-describing & $.15^{\dagger}$ & -.03 & $-.29^{* *}$ & $-.22^{*}$ & -.06 & -.08 & $-.20^{*}$ & $-.26^{* *}$ & $-.31^{* *}$ & $.53^{* *}$ & $.37^{* *}$ & $.25^{* *}$ & $.19^{*}$ & 1 & & \\
\hline 15. FFMQ-awareness & $.23^{*}$ & $-.22^{*}$ & $-.41^{* *}$ & $-.22^{*}$ & $-.20^{*}$ & .05 & $-.35^{* *}$ & $-.32^{* *}$ & $-.41^{* *}$ & $.60^{* *}$ & $.70^{* *}$ & .08 & $.35^{* *}$ & $.32^{* *}$ & 1 & \\
\hline 16. FFMQ-nonreactance & $.19^{*}$ & -.12 & $-.32^{* *}$ & $-.18^{*}$ & $-.24^{* *}$ & $-.20^{*}$ & -.07 & $-.31^{* *}$ & $-.35^{* *}$ & $.47^{* *}$ & $.17^{\dagger}$ & $.48^{* *}$ & .01 & $.21^{*}$ & $.16^{\dagger}$ & 1 \\
\hline
\end{tabular}

Note. ${ }^{* *} p<0.01 ;{ }^{*} p<0.05 ; \dagger p<0.10$. BDI-II = Beck Depression Inventory-II; RRS-R = Ruminative Response Scale-Reflection; RRS-B = Ruminative Response Scale-Reflection; DAS-SF1 = Dysfunctional Attitudes Scale-Short Form 1; STAI-S = State-Trait Anxiety Inventory - State; STAI-T = State-Trait Anxiety Inventory - Trait; BAI = Beck Anxiety Inventory; PANAS- PA= Positive and Negative Affect Schedule - Positive Affect; PANAS - NA= Positive and Negative Affect Schedule - Negative Affect; MAAS - Mindful Attention Awareness Scale; FFMQ = Five Facet Mindfulness Questionnaire. 
Table 11. Study 2 FD Ratio and Difference Score Correlations

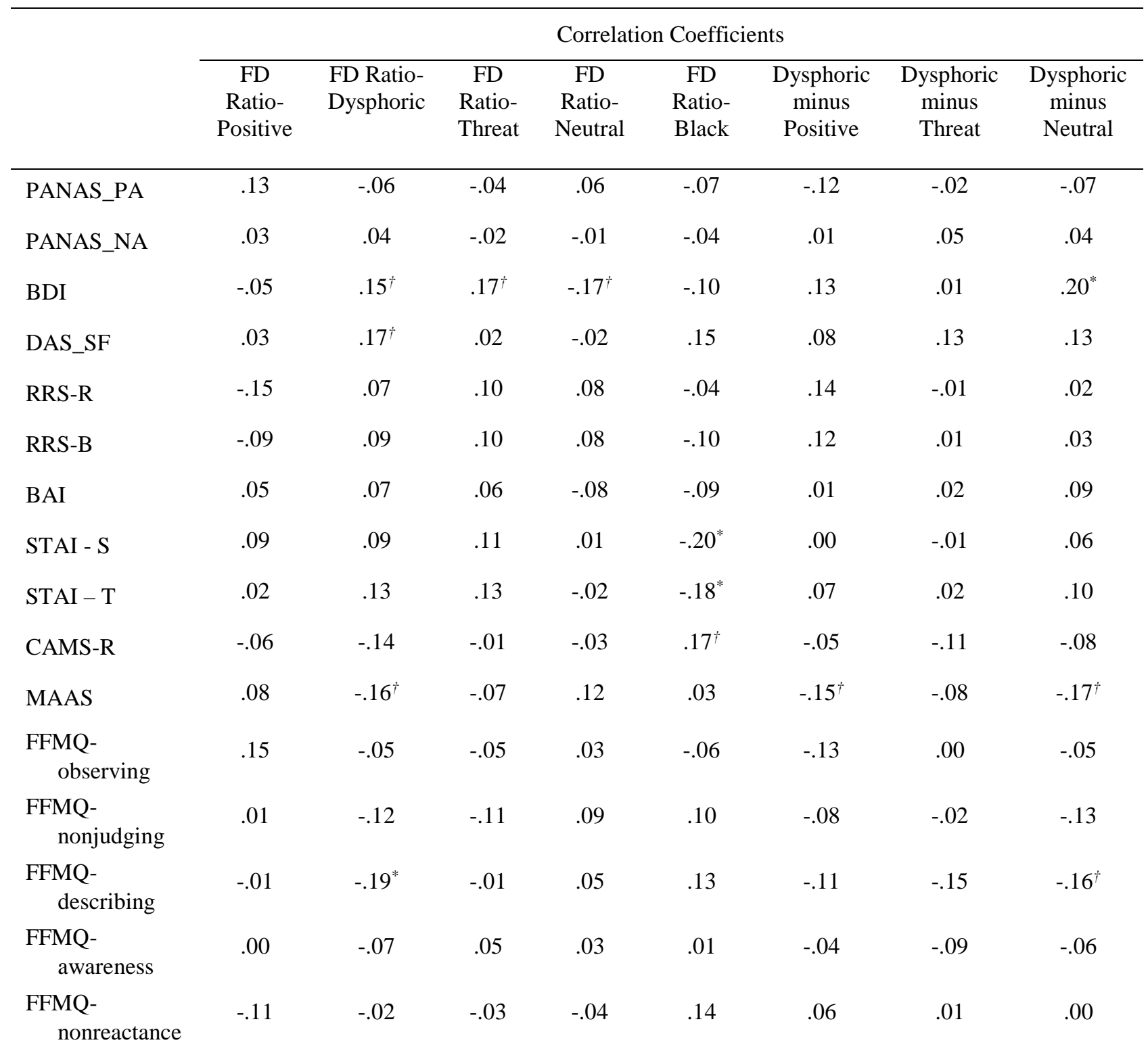

Note. ${ }^{* *} p<0.01 ; *<<0.05 ; \dagger p<0.10$. PANAS- PA= Positive and Negative Affect Schedule - Positive Affect; PANAS - NA= Positive and Negative Affect Schedule - Negative Affect; BDI-II = Beck Depression Inventory-II; DAS-SF1 = Dysfunctional Attitudes Scale-Short Form-1; RRS-R = Ruminative Response Scale-Reflection; RRS-B $=$ Ruminative Response Scale-Brooding; BAI = Beck Anxiety Inventory; STAI-State $=$ State-Trait Anxiety Inventory - Trait; CAMSR = Cognitive and Affective Mindfulness Scale Revised; MAAS - Mindful Attention Awareness Scale; FFMQ = Five Facet Mindfulness Questionnaire; FD = Fixation Duration. 
Table 12. Study 2 FC Ratio and Difference Score Correlations

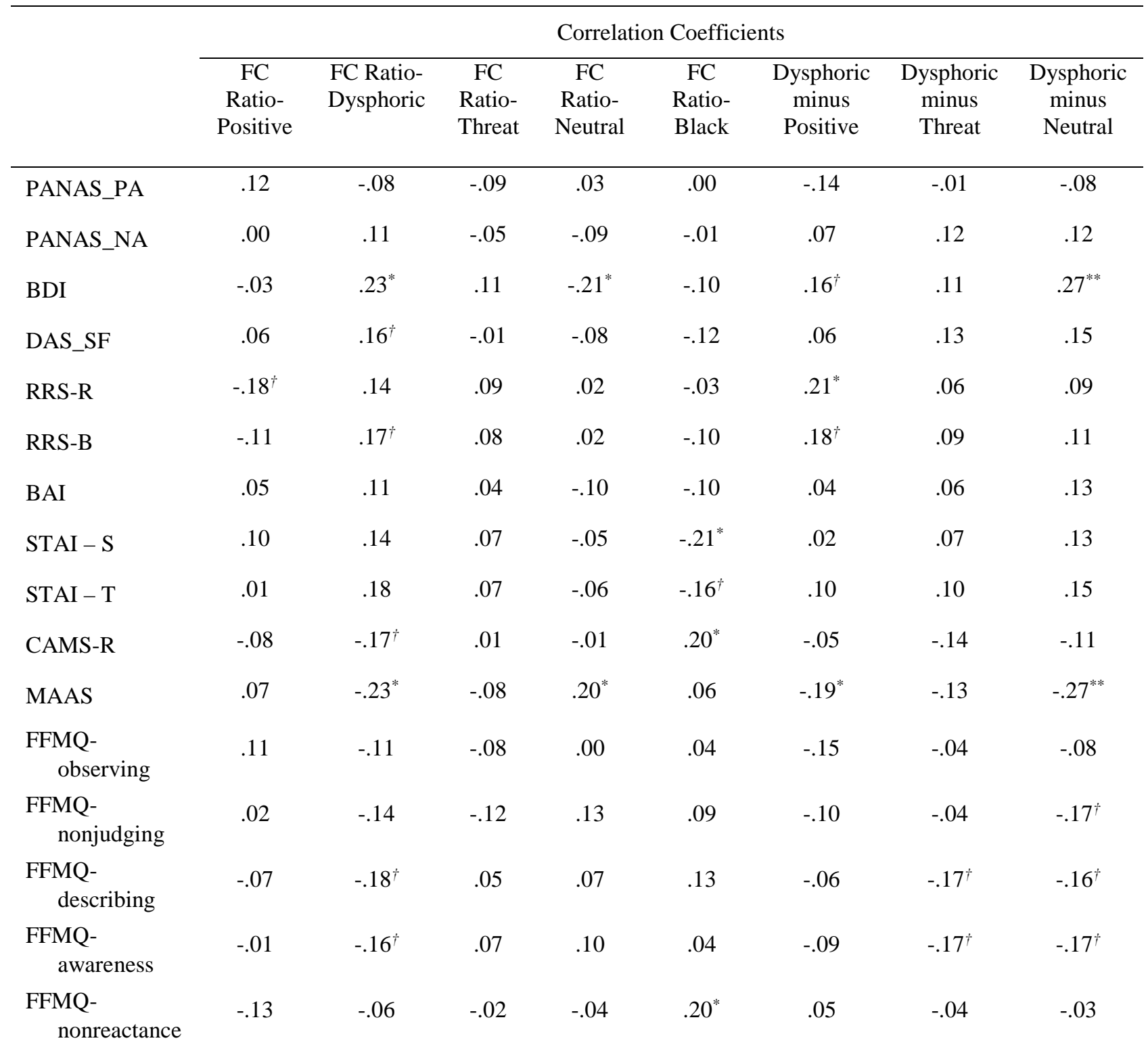

Note. ${ }^{* *} p<0.01 ; *<<0.05 ; \dagger p<0.10$. PANAS- PA= Positive and Negative Affect Schedule - Positive Affect; PANAS - NA= Positive and Negative Affect Schedule - Negative Affect; BDI-II = Beck Depression Inventory-II; DAS-SF1 = Dysfunctional Attitudes Scale-Short Form-1; RRS-R = Ruminative Response Scale-Reflection; RRS-B $=$ Ruminative Response Scale-Brooding; BAI = Beck Anxiety Inventory; STAI-State $=$ State-Trait Anxiety Inventory - Trait; CAMSR = Cognitive and Affective Mindfulness Scale Revised; MAAS - Mindful Attention Awareness Scale; FFMQ = Five Facet Mindfulness Questionnaire; FC = Fixation Count. 
Table 13. Study 2 FFT Correlations

\begin{tabular}{|c|c|c|c|c|}
\hline & \multicolumn{4}{|c|}{ Correlation Coefficients } \\
\hline & $\begin{array}{l}\text { FFT - } \\
\text { Positive }\end{array}$ & FFT - Dysphoric & $\begin{array}{l}\text { FFT- } \\
\text { Threat }\end{array}$ & $\begin{array}{l}\text { FFT - } \\
\text { Neutral }\end{array}$ \\
\hline PANAS_PA & .07 & -.10 & .10 & -.09 \\
\hline PANAS_NA & .04 & -.04 & -.01 & -.14 \\
\hline BDI & .15 & .09 & .08 & -.01 \\
\hline DAS_SF & -.02 & -.05 & -.01 & -.02 \\
\hline RRS-R & .16 & -.11 & -.01 & -.12 \\
\hline RRS-B & $.25^{* *}$ & $.20^{*}$ & -.01 & -.01 \\
\hline BAI & .03 & -.10 & -.10 & -.13 \\
\hline STAI - S & .10 & .02 & -.10 & -.04 \\
\hline STAI - T & .08 & .06 & -.04 & -.05 \\
\hline CAMS-R & .04 & -.02 & .09 & .06 \\
\hline MAAS & -.11 & .06 & .04 & -.05 \\
\hline FFMQ-observing & -.04 & $-.16^{\dagger}$ & $-.25^{* *}$ & -.09 \\
\hline FFMQ-nonjudging & -.09 & -.11 & -.08 & .13 \\
\hline FFMQ-describing & -.11 & -.15 & .04 & -.01 \\
\hline FFMQ-awareness & -.01 & .10 & .14 & .08 \\
\hline FFMQ-nonreactance & .03 & -.05 & -.09 & .00 \\
\hline
\end{tabular}

Note: ${ }^{* *} p<0.01 ; * p<0.05 ; \dagger p<0.10$. PANAS- PA= Positive and Negative Affect Schedule - Positive Affect; PANAS - NA= Positive and Negative Affect Schedule - Negative Affect; BDI-II = Beck Depression Inventory-II; DAS-SF1 = Dysfunctional Attitudes Scale-Short Form-1; RRS-R = Ruminative Response Scale-Reflection; RRS-B = Ruminative Response Scale-Brooding; BAI = Beck Anxiety Inventory; STAI-State = State-Trait Anxiety Inventory - Trait; CAMSR = Cognitive and Affective Mindfulness Scale Revised; MAAS - Mindful Attention Awareness Scale; FFMQ = Five Facet Mindfulness Questionnaire; FFT = First Fixation Type. 
Figure 1. Study 2 Fixation Duration Scores by Condition

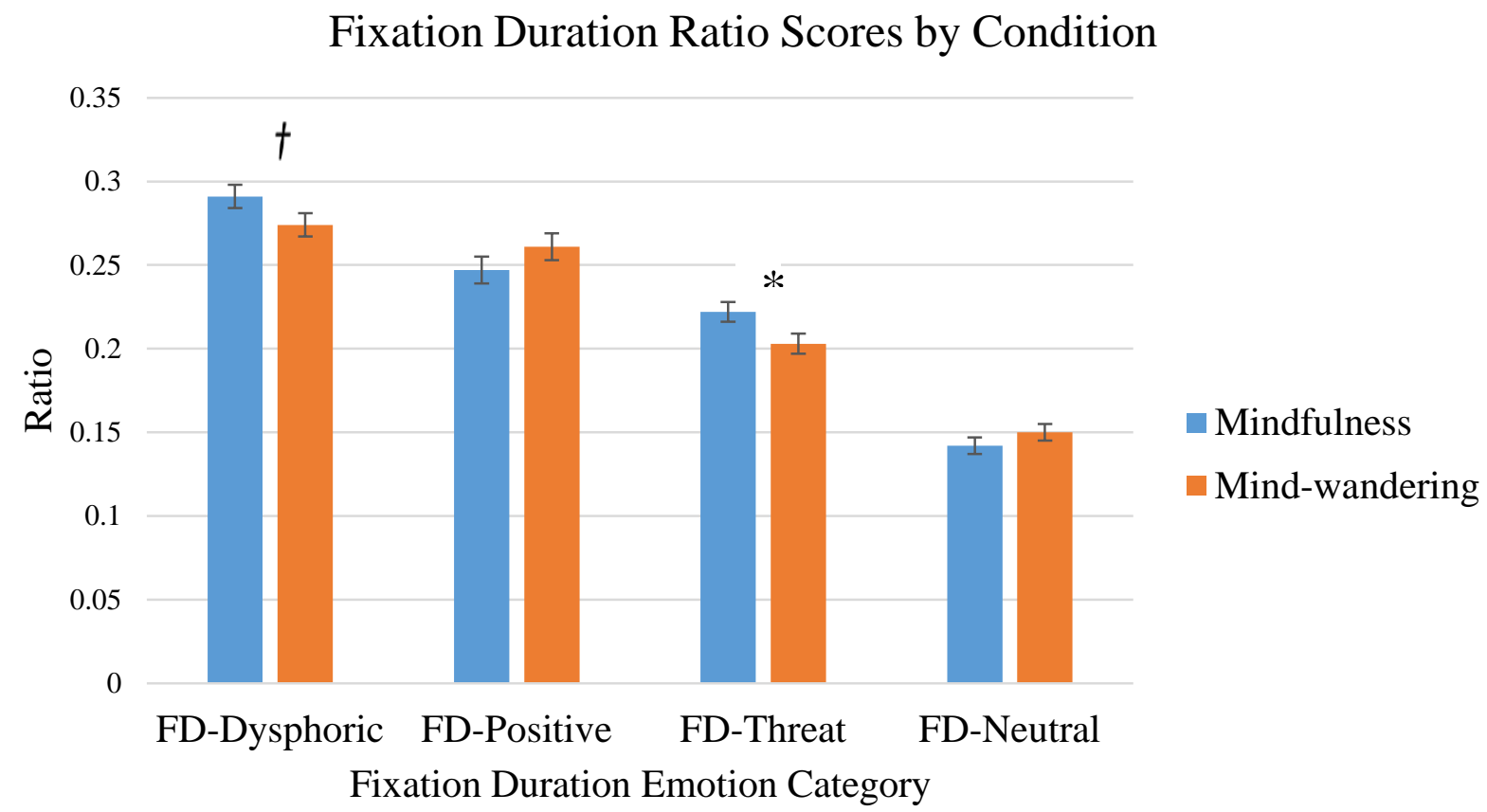


Appendix A

Dysphoric Example Images
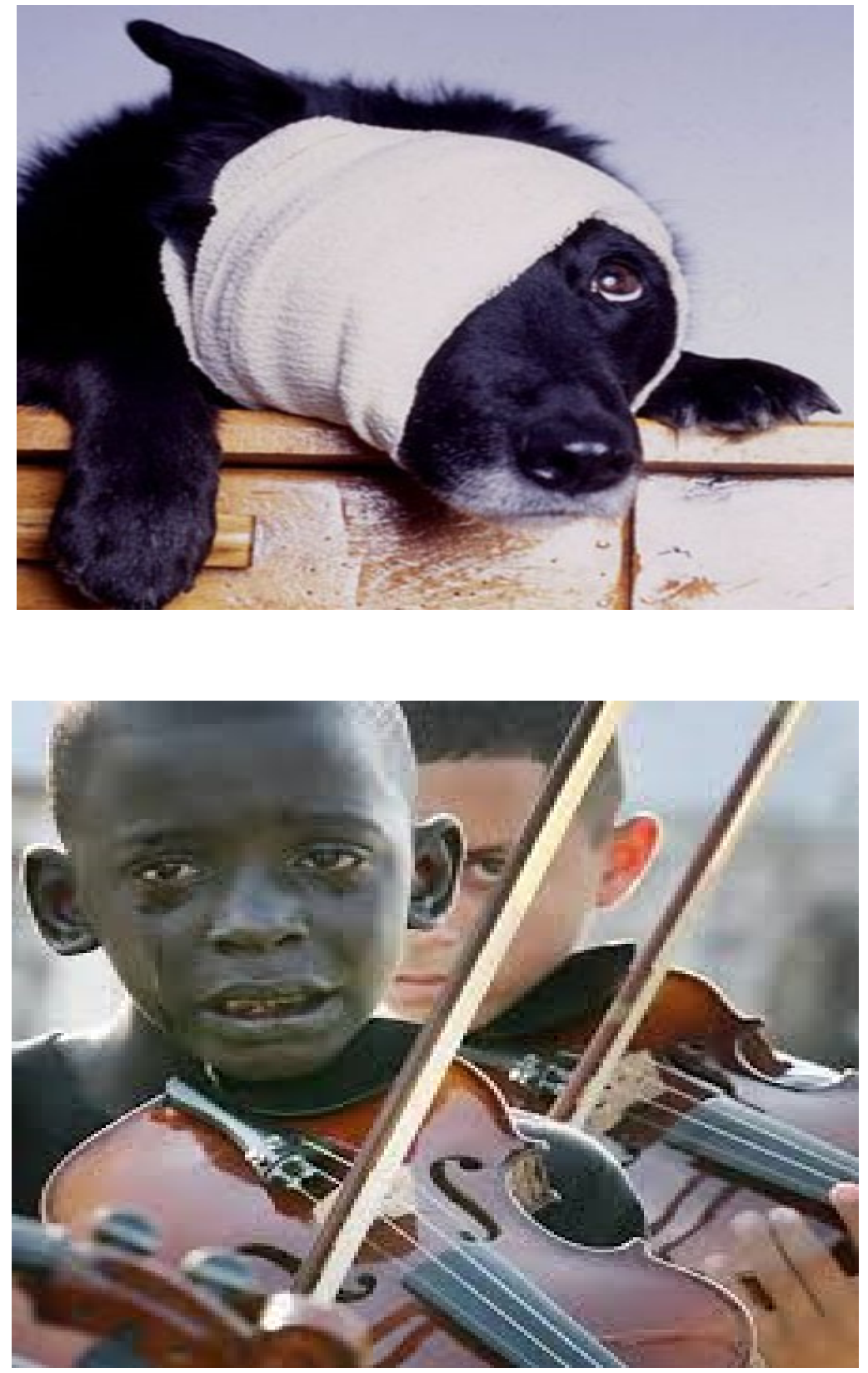

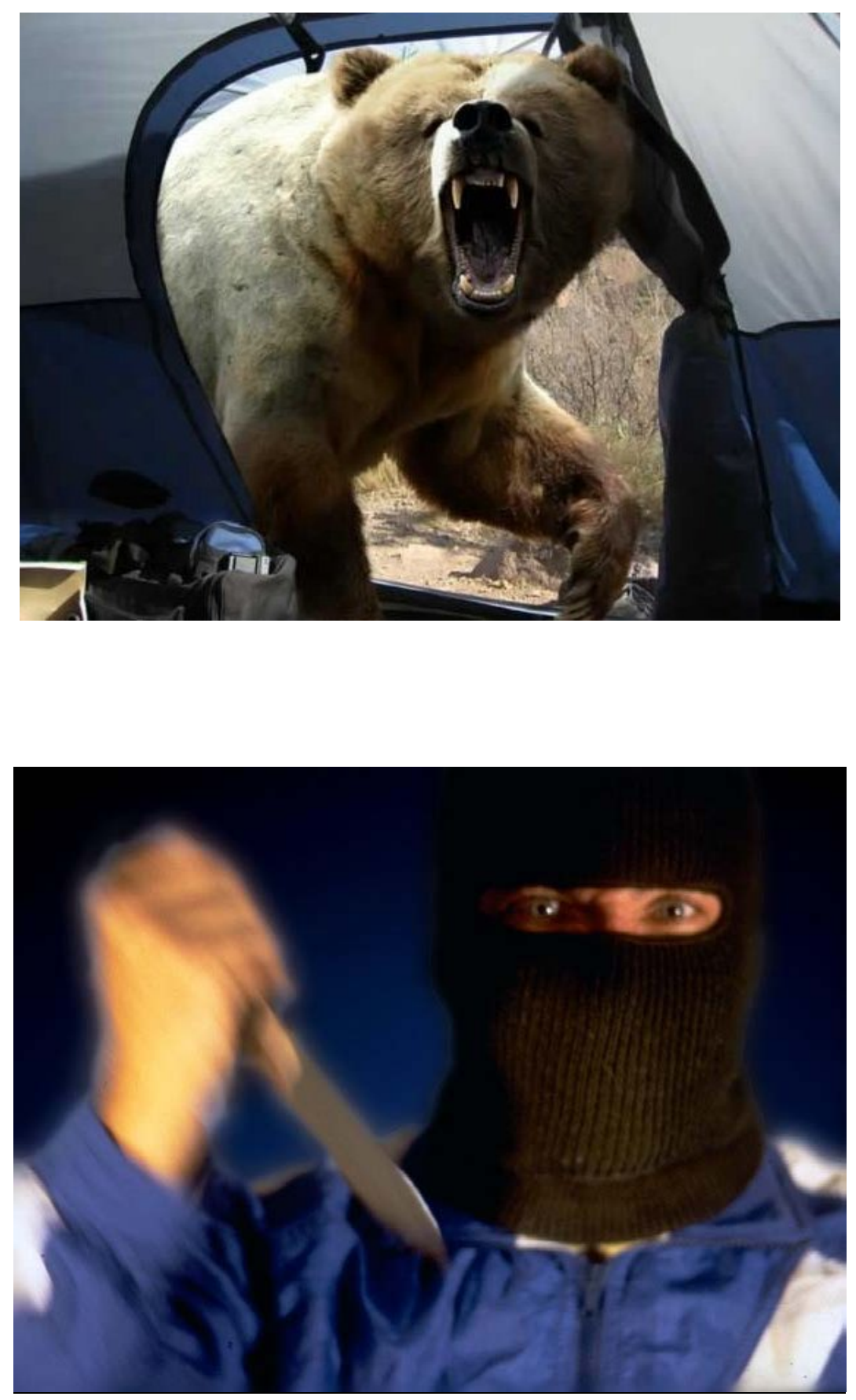
Neutral Example Images
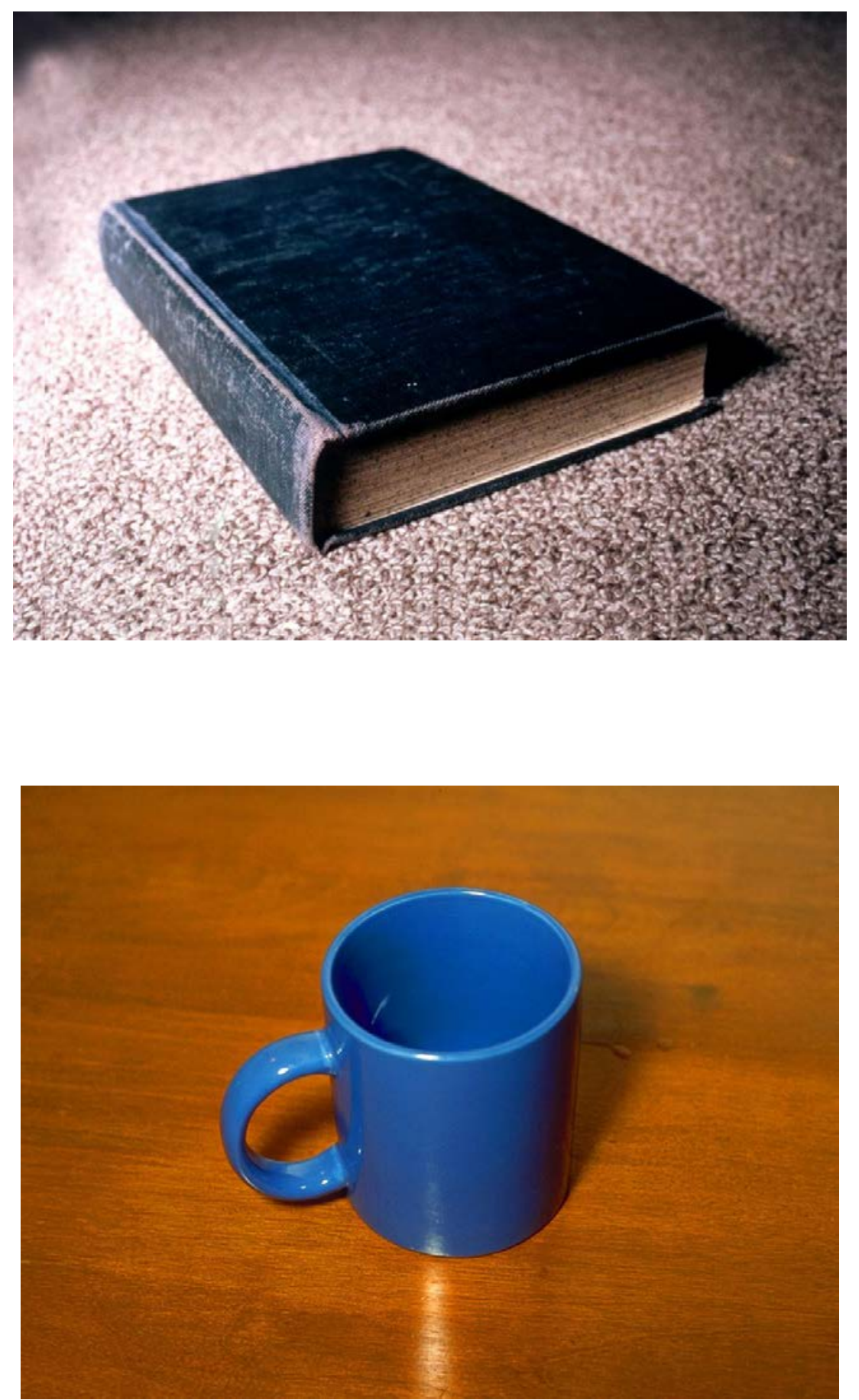
Positive Example Images
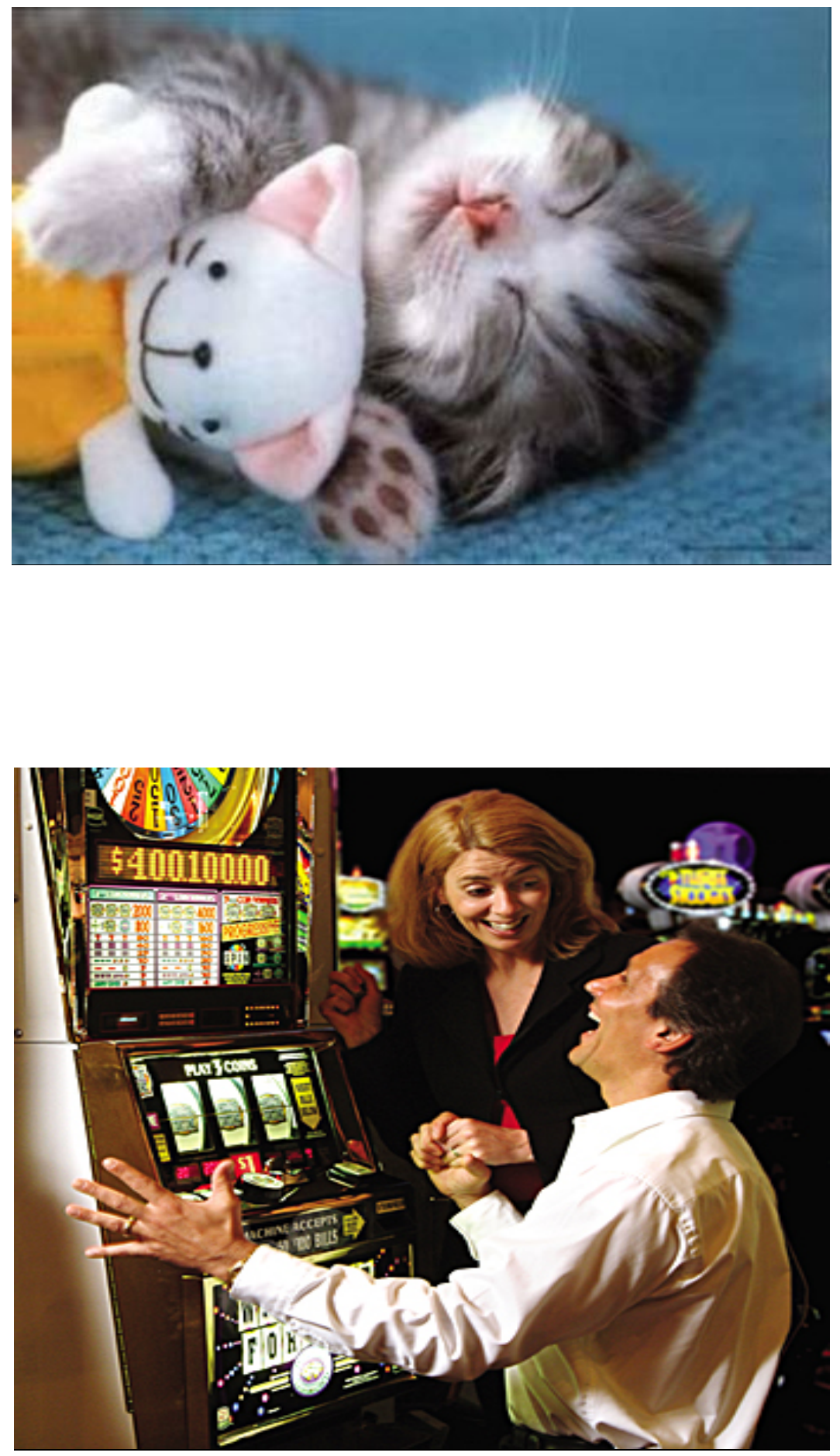
Appendix B

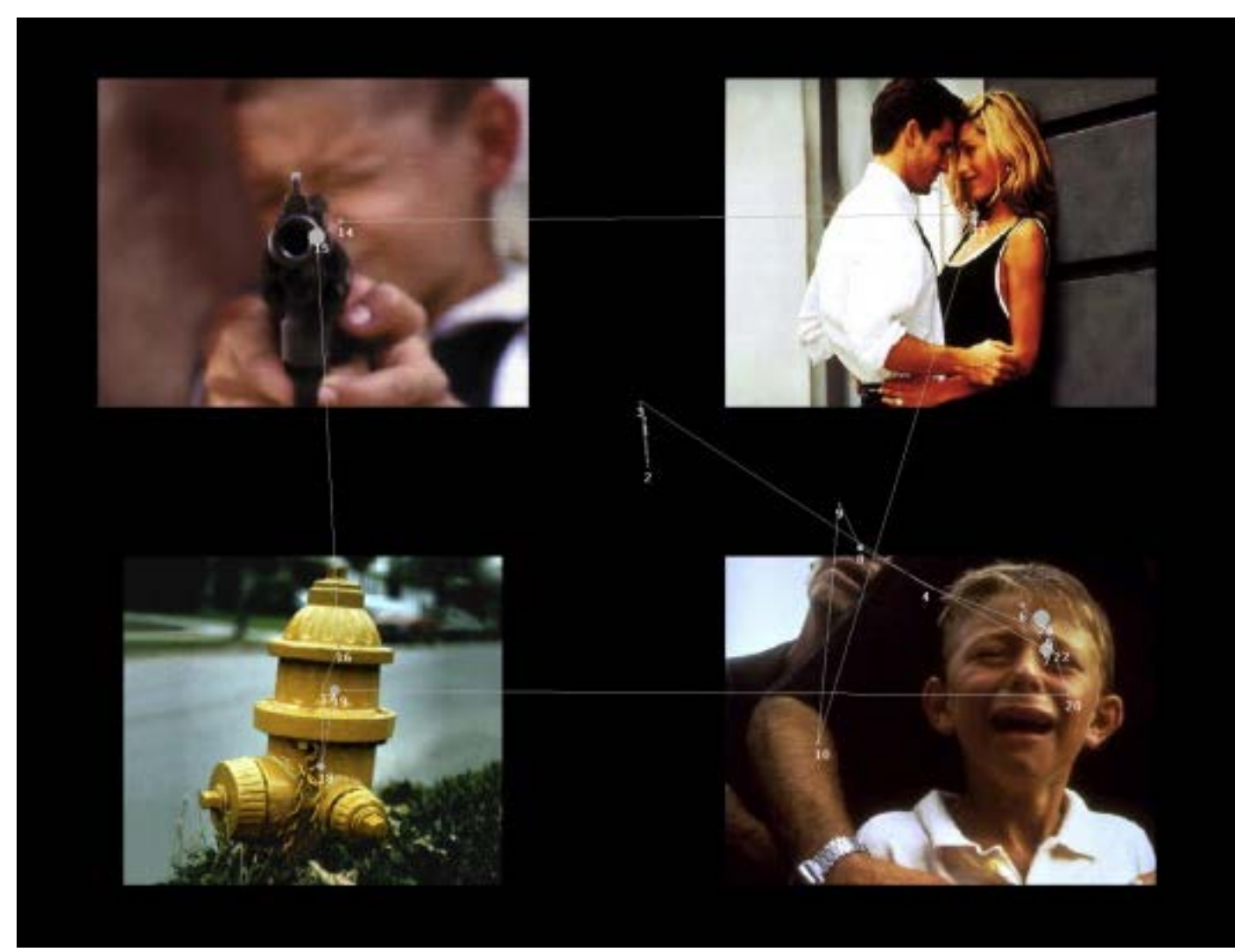




\section{Appendix C}

\section{Rumination Scale Treynor, Gonzalez, \& Nolen-Hoeksema, 2003}

People think and do many different things when they feel depressed. Please read each of the items below and indicate whether you almost never, sometimes, often, or almost always think or do each one when you feel down, sad, or depressed. Please indicate what you generally do, not what you think you should do.

1 almost never 2 sometimes 3 often 4 almost always

1. Think about how alone you feel

2. Think "I won't be able to do my job if I don't snap out of this"

3. Think about your feelings of fatigue and achiness

4. Think about how hard it is to concentrate

5. Think "What am I doing to deserve this?"

6. Think about how passive and unmotivated you feel.

7. Analyze recent events to try to understand why you are depressed

8. Think about how you don't seem to feel anything anymore

9. Think "Why can't I get going?"

10. Think "Why do I always react this way?"

11. Go away by yourself and think about why you feel this way

12. Write down what you are thinking about and analyze it

13. Think about a recent situation, wishing it had gone better

14. Think "I won't be able to concentrate if I keep feeling this way."

15. Think "Why do I have problems other people don't have?"

16. Think "Why can't I handle things better?"

17. Think about how sad you feel.

18. Think about all your shortcomings, failings, faults, mistakes

19. Think about how you don't feel up to doing anything

20. Analyze your personality to try to understand why you are depressed

21. Go someplace alone to think about your feelings

22. Think about how angry you are with yourself 


\section{DAS-SF1}

\section{Beevers, Strong, Meyer, Pilkonis, \& Miller, 2007}

The sentences below describe people's attitudes. Circle the number which best describes how much each sentence describes your attitude. Your answer should describe the way you think most of the time.

Totally Agree

1

$$
\begin{gathered}
\text { Agree } \\
2
\end{gathered}
$$

\author{
Disagree \\ 3
}

\author{
Totally Disagree \\ 4
}

1. If I don't set the highest standards for myself, I am likely to end up a second-rate person.

2. My value as a person depends greatly on what others think of me.

3. People will probably think less of me if I make a mistake.

4. I am nothing if a person I love doesn't love me.

5. If other people know what you are really like, they will think less of you.

6. If I fail at my work, then I am a failure as a person.

7. My happiness depends more on other people than it does me.

8. I cannot be happy unless most people I know admire me.

9. It is best to give up your own interests in order to please other people. 
State and Trait Anxiety Inventory

(Spielberger, Gorsuch, Lushene, Vagg, \& Jacobs 1983)

The following is a list of statements which people have used to describe themselves. We want you to indicate how you feel right now, that is, at this moment using the rating scale provided. There are no right or wrong answers. Do not spend too much time on any one statement, just give the answer which seems to describe your present feelings best.

\begin{tabular}{|l|c|c|c|c|}
\hline & $\begin{array}{c}\text { Not at } \\
\text { all }\end{array}$ & $\begin{array}{c}\text { Somew } \\
\text { hat }\end{array}$ & $\begin{array}{c}\text { Moderat } \\
\text { ely so }\end{array}$ & $\begin{array}{c}\text { Very } \\
\text { much } \\
\text { so }\end{array}$ \\
\hline 1. I feel calm & 1 & 2 & 3 & 4 \\
\hline 2. I feel secure & 1 & 2 & 3 & 4 \\
\hline 3. I am tense & 1 & 2 & 3 & 4 \\
\hline 4. I feel strained & 1 & 2 & 3 & 4 \\
\hline 5. I feel at ease & 1 & 2 & 3 & 4 \\
\hline 6. I feel upset & 1 & 2 & 3 & 4 \\
\hline 7. I am presently working over possible misfortunes & 1 & 2 & 3 & 4 \\
\hline 8. I feel satisfied & 1 & 2 & 3 & 4 \\
\hline 9. I feel heighten & 1 & 2 & 3 & 4 \\
\hline 10. I feel comfortable & 1 & 2 & 3 & 4 \\
\hline 11. I feel self-confident & 1 & 2 & 3 & 4 \\
\hline 12. I feel nervous & 1 & 2 & 3 & 4 \\
\hline 13. I am jittery & 1 & 2 & 3 & 4 \\
\hline 14. I feel indecisive & 1 & 2 & 3 & 4 \\
\hline 15. I am relaxed & & 2 & 3 & 4 \\
\hline 16. I feel content & 1 & & 3 & 4 \\
\hline
\end{tabular}




\begin{tabular}{|l|c|c|c|c|}
\hline 17. I am worried & 1 & 2 & 3 & 4 \\
\hline 18. I feel confused & 1 & 2 & 3 & 4 \\
\hline 19. I feel steady & 1 & 2 & 3 & 4 \\
\hline 20. I feel pleasant & 1 & 2 & 3 & 4 \\
\hline
\end{tabular}

Now please indicate how you generally feel using the rating scale provided. Again, there are no right or wrong answers. Do not spend too much time on any one statement just give the answer which seems to describe how you generally feel.

\begin{tabular}{|l|c|c|c|c|}
\hline & $\begin{array}{c}\text { Not at } \\
\text { all }\end{array}$ & $\begin{array}{c}\text { Somew } \\
\text { hat }\end{array}$ & $\begin{array}{c}\text { Moderat } \\
\text { ely so } \\
\text { much } \\
\text { so }\end{array}$ & $\begin{array}{c}\text { Very } \\
\text { 21. I feel pleasant }\end{array}$ \\
\hline 22. I feel nervous and restless & 1 & 2 & 3 & 4 \\
\hline 23. I feel satisfied with myself & 1 & 2 & 3 & 4 \\
\hline 24. I wish I could be as happy as others seem to be & 1 & 2 & 3 & 4 \\
\hline 25. I feel like a failure & 1 & 2 & 3 & 4 \\
\hline 26. I feel rested & 1 & 2 & 3 & 4 \\
\hline 27. I am “calm, cool, and collected” & 1 & 2 & 3 & 4 \\
\hline 28. I feel that difficulties are piling up so that I cannot \\
overcome them
\end{tabular}




\begin{tabular}{|l|c|c|c|c|}
\hline 32. I lack self-confidence & 1 & 2 & 3 & 4 \\
\hline 33. I feel secure & 1 & 2 & 3 & 4 \\
\hline 34. I make decisions easily & 1 & 2 & 3 & 4 \\
\hline 35. I feel inadequate & 1 & 2 & 3 & 4 \\
\hline 36. I am content & 1 & 2 & 3 & 4 \\
\hline $\begin{array}{l}\text { 37. Some unimportant thought runs through my mind } \\
\text { and bothers me }\end{array}$ & 1 & 2 & 3 & 4 \\
\hline $\begin{array}{l}\text { 38. I take disappointments so keenly that I can't pull } \\
\text { them out of my mind }\end{array}$ & 1 & 2 & 3 & 4 \\
\hline $\begin{array}{l}\text { 39. I am a steady person } \\
\text { 40. I get in a state of tension or turmoil as I think over } \\
\text { my recent concerns and interests }\end{array}$ & 1 & 2 & 3 & 4 \\
\hline
\end{tabular}




\section{The Positive Affect Negative Affect Schedule (PANAS; Watson, Clark \& Tellegen, 1988)}

Instructions: This scale consists of a number of words that describe different feelings and emotions. Read each item and then mark the appropriate answer in the space next to that word. Indicate to what extent you feel this way RIGHT NOW, that is, at this very moment. Use the following scale to record your answers:

\begin{tabular}{|c|c|c|c|c|}
\hline $\begin{array}{l}\text { very slightly } \\
\text { or not at all }\end{array}$ & a little & moderately & quite a bit & extremely \\
\hline 1 & 2 & 3 & 4 & 5 \\
\hline interested & \multicolumn{4}{|c|}{ irritable } \\
\hline distressed & \multicolumn{4}{|c|}{ alert } \\
\hline excited & \multicolumn{4}{|c|}{ ashamed } \\
\hline upset & \multicolumn{4}{|c|}{ inspired } \\
\hline strong & \multicolumn{4}{|c|}{ nervous } \\
\hline guilty & \multicolumn{4}{|c|}{ determined } \\
\hline scared & \multicolumn{4}{|c|}{ attentive } \\
\hline hostile & \multicolumn{4}{|c|}{ jittery } \\
\hline enthusiastic & \multicolumn{4}{|c|}{ active } \\
\hline proud & \multicolumn{4}{|c|}{ afraid } \\
\hline
\end{tabular}




\section{Mindful Attention Awareness Scale (Brown and Ryan, 2003)}

Instructions: Below is a collection of statements about your everyday experience. Using the 1-6 scale below, please indicate how frequently or infrequently you currently have each experience. Please answer according to what really reflects your experience rather than what you think your experience should be. Please treat each item separately from every other item.

$\begin{array}{cccccc}1 & 2 & 3 & 4 & 5 & 6 \\ \text { Almost } & \text { Very } & \text { Somewhat } & \text { Somewhat } & \text { Very } & \text { Almost } \\ \text { Always } & \text { Frequently } & \text { Frequently } & \text { Infrequently } & \text { Infrequently } & \text { Never }\end{array}$

I could be experiencing some emotion and not be conscious of it until some time later.

$\begin{array}{llllll}1 & 2 & 3 & 4 & 5 & 6\end{array}$

I break or spill things because of carelessness, not paying attention, or thinking of something else.

I find it difficult to stay focused on what's happening in the present.

$\begin{array}{llllll}1 & 2 & 3 & 4 & 5 & 6\end{array}$

I tend to walk quickly to get where I'm going without paying attention to what I experience along the way.

$\begin{array}{llllll}1 & 2 & 3 & 4 & 5 & 6\end{array}$

I tend not to notice feelings of physical tension or discomfort until they really grab my attention.

$\begin{array}{llllll}1 & 2 & 3 & 4 & 5 & 6\end{array}$

I forget a person's name almost as soon as I've been told it for the first time.

$\begin{array}{llllll}1 & 2 & 3 & 4 & 5 & 6\end{array}$

It seems I am “running on automatic,” without much awareness of what I'm doing.

$\begin{array}{llllll}1 & 2 & 3 & 4 & 5 & 6\end{array}$

I rush through activities without being really attentive to them.

I get so focused on the goal I want to achieve that I lose touch with what I'm doing right now to get there.

$\begin{array}{llllll}1 & 2 & 3 & 4 & 5 & 6\end{array}$

I do jobs or tasks automatically, without being aware of what I'm doing.

I find myself listening to someone with one ear, doing something else at the same time. 


\begin{tabular}{|lrrrrrrr}
\hline $\begin{array}{l}\text { I drive places on "automatic pilot” and then wonder why I } \\
\text { went there. }\end{array}$ & 1 & 2 & 3 & 4 & 5 & 6 \\
I find myself preoccupied with the future or the past. & 1 & 2 & 3 & 4 & 5 & 6 \\
I find myself doing things without paying attention. & 1 & 2 & 3 & 4 & 5 & 6 \\
I snack without being aware that I'm eating. & 1 & 2 & 3 & 4 & 5 & 6 \\
\hline
\end{tabular}




\section{Cognitive and Affective Mindfulness Scale - Revised \\ (Feldman, Hayes, Kumar, Greeson \& Laurenceau, 2007)}

\begin{tabular}{|c|c|c|c|c|c|}
\hline \multicolumn{2}{|r|}{ Please respond to each item by marking one box per row } & \multirow{2}{*}{$\begin{array}{c}\begin{array}{c}\text { Rarely/Not } \\
\text { at All }\end{array} \\
\square \\
\square \\
\end{array}$} & \multirow{2}{*}{$\begin{array}{c}\text { Sometimes } \\
\square \\
2\end{array}$} & \multirow{2}{*}{$\frac{\square_{3}}{\square_{3}}$} & \multirow{2}{*}{$\begin{array}{c}\begin{array}{c}\text { Almost } \\
\text { Always }\end{array} \\
\frac{\square}{4}\end{array}$} \\
\hline CAMS-R1 & It is easy for me to concentrate on what I am doing. & & & & \\
\hline CAMS-R3 & I can tolerate emotional pain. & $\begin{array}{l}\square \\
1 \\
\end{array}$ & $\begin{aligned} \square \\
2 \\
\end{aligned}$ & $\begin{array}{ll}\square \\
3\end{array}$ & $\begin{aligned} \square \\
4 \\
\end{aligned}$ \\
\hline CAMS-R4 & I can accept things I cannot change. & 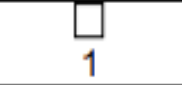 & 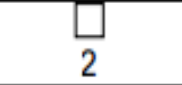 & $\square$ & $\square$ \\
\hline CAMS-R5 & $\begin{array}{l}\text { I can usually describe how I feel at the moment in } \\
\text { considerable detail. }\end{array}$ & $\begin{array}{l}\square \\
\end{array}$ & $\begin{array}{l}\square \\
\end{array}$ & $\square$ & 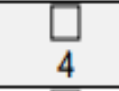 \\
\hline CAMS-R6 & I am easily distracted. (R) & $\begin{array}{l}\square \\
\end{array}$ & 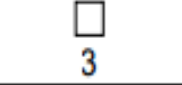 & $\begin{array}{l}\square \\
2\end{array}$ & $\begin{array}{l}\square \\
1\end{array}$ \\
\hline CAMS-R8 & $\begin{array}{l}\text { It's easy for me to keep track of my thoughts and } \\
\text { feelings. }\end{array}$ & $\begin{array}{l}\square \\
1\end{array}$ & $\square$ & $\square$ & $\square$ \\
\hline CAMS-R9 & I try to notice my thoughts without judging them. & $\begin{array}{l}\square \\
1\end{array}$ & $\begin{array}{l}\square \\
\end{array}$ & $\begin{array}{l}\square \\
\end{array}$ & $\begin{array}{l}\square \\
\end{array}$ \\
\hline$\underset{\text { R10 }}{\text { CAMS. }}$ & I am able to accept the thoughts and feelings I have. & $\begin{array}{l}\square \\
\end{array}$ & $\square$ & $\square$ & $\square$ \\
\hline $\begin{array}{c}\text { CAMS. } \\
\text { R11 }\end{array}$ & I am able to focus on the present moment. & $\square$ & $\square$ & $\square$ & $\square$ \\
\hline $\begin{array}{c}\text { CAMS. } \\
\text { R12 }\end{array}$ & $\begin{array}{l}\text { I am able to pay close attention to one thing for a long } \\
\text { period of time. }\end{array}$ & $\begin{array}{ll}\square \\
1\end{array}$ & $\square$ & 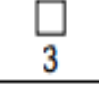 & $\frac{\square}{4}$ \\
\hline
\end{tabular}




\section{Beck Anxiety Inventory}

\section{(BAI; Beck, Epstein, Brown, \& Steer, 1988)}

Below is a list of common symptoms of anxiety. Please carefully read each item in the list. Indicate how much you have been bothered by that symptom during the past month, including today, by circling the number in the corresponding space in the column next to each symptom.

\begin{tabular}{|l|c|c|c|c|}
\hline & Not At All & $\begin{array}{l}\text { Mildly but it } \\
\text { didn't bother me } \\
\text { much. }\end{array}$ & $\begin{array}{l}\text { Moderately - it } \\
\text { wasn't pleasant at } \\
\text { times }\end{array}$ & $\begin{array}{l}\text { Severely - it } \\
\text { bothered me a lot }\end{array}$ \\
\hline Numbness or tingling & 0 & 1 & 2 & 3 \\
\hline Feeling hot & 0 & 1 & 2 & 3 \\
\hline Wobbliness in legs & 0 & 1 & 2 & 3 \\
\hline Unable to relax & 0 & 1 & 2 & 3 \\
\hline $\begin{array}{l}\text { Fear of worst } \\
\text { happening }\end{array}$ & 0 & 1 & 2 & 3 \\
\hline Dizzy or lightheaded & 0 & 1 & 2 & 3 \\
\hline Heart pounding/racing & 0 & 1 & 2 & 3 \\
\hline Unsteady & 0 & 1 & 2 & 3 \\
\hline Terrified or afraid & 0 & 1 & 2 & 3 \\
\hline Nervous & 0 & 1 & 2 & 3 \\
\hline Feeling of choking & 0 & 1 & 2 & 3 \\
\hline Hands trembling & 0 & 1 & 2 & 3 \\
\hline Shaky / unsteady & 0 & 1 & 2 & 3 \\
\hline Fear of losing control & 0 & 1 & 2 & 3 \\
\hline Difficulty in breathing & 0 & 1 & 2 & 3 \\
\hline Fear of dying & 0 & 1 & 2 & 3 \\
\hline Scared & 0 & 1 & 2 & 3 \\
\hline Indigestion & 0 & 1 & 2 & 3 \\
\hline Faint / lightheaded & 0 & 1 & 2 & 3 \\
\hline Face flushed & 0 & 1 & 2 & 3 \\
\hline Hot/cold sweats & 0 & 1 & 2 & 3 \\
\hline
\end{tabular}




\section{Five Facet Mindfulness Questionnaire \\ (Baer, Smith, Hopkins, Krietenmeyer \& Toney, 2006)}

Please rate each of the following statements using the scale provided. Write the number in the blank that best describes your own opinion of what is generally true for you.

$\begin{array}{lcccc}1 & 2 & 3 & 4 & 5 \\ \text { Never or } & \text { Rarely true } & \text { Sometimes true } & \text { often true } & \text { very often } \\ \text { Rarely true } & & & & \text { always true }\end{array}$

1. When I'm walking, I deliberately notice the sensations of my body moving.

2. I'm good at finding words to describe my feelings.

3. I criticize myself for having irrational or inappropriate emotions.

4. I perceive my feelings and emotions without having to react to them.

5. When I do things, my mind wanders off and I'm easily distracted.

6. When I take a shower or bath, I stay alert to the sensations of water on my body.

7. I can easily put my beliefs, opinions, and expectations into words.

8. I don't pay attention to what I'm doing because I'm daydreaming, worrying, or

otherwise distracted.

9. I watch my feelings without getting lost in them.

10. I tell myself I shouldn't be feeling the way I'm feeling.

11. I notice how foods and drinks affect my thoughts, bodily sensations, and emotions.

12. It's hard for me to find the words to describe what I'm thinking.

13. I am easily distracted.

14. I believe some of my thoughts are abnormal or bad and I shouldn't think that way.

15. I pay attention to sensations, such as the wind in my hair or sun on my face.

16. I have trouble thinking of the right words to express how I feel about things

17. I make judgments about whether my thoughts are good or bad.

18. I find it difficult to stay focused on what's happening in the present.

19. When I have distressing thoughts or images, I "step back" and am aware of the

thought or image without getting taken over by it.

20. I pay attention to sounds, such as clocks ticking, birds chirping, or cars passing.

21. In difficult situations, I can pause without immediately reacting.

22. When I have a sensation in my body, it's difficult for me to describe it because

I can't find the right words.

23. It seems I am "running on automatic" without much awareness of what I'm doing.

24. When I have distressing thoughts or images, I feel calm soon after.

25. I tell myself that I shouldn't be thinking the way I'm thinking.

26. I notice the smells and aromas of things.

27. Even when I'm feeling terribly upset, I can find a way to put it into words.

28. I rush through activities without being really attentive to them.

29. When I have distressing thoughts or images I am able just to notice them without reacting.

30. I think some of my emotions are bad or inappropriate and I shouldn't feel them. 
31. I notice visual elements in art or nature, such as colors, shapes, textures, or patterns of light and shadow.

32. My natural tendency is to put my experiences into words.

33. When I have distressing thoughts or images, I just notice them and let them go.

34. I do jobs or tasks automatically without being aware of what I'm doing.

35. When I have distressing thoughts or images, I judge myself as good or bad, depending what the thought/image is about.

36. I pay attention to how my emotions affect my thoughts and behavior.

37. I can usually describe how I feel at the moment in considerable detail.

38. I find myself doing things without paying attention.

39. I disapprove of myself when I have irrational ideas. 


\section{Demographics Questions}

Gender: $\quad$ Male $\quad$ Female

Age:

Year in College:

Freshman

Sophomore

Junior

Senior

Other:

Sexual orientation:

Straight/Heterosexual

Lesbian/Homosexual Female

Gay/Homosexual Male

Bi-sexual

Transgendered

Other

Political orientation:

Very conservative

Conservative

Moderate

Liberal

Very liberal

What political party best represents your beliefs?

_Democrat __ Republican ___Libertarian __ Independent __ Other

Marital Status:

\begin{tabular}{l} 
Single \\
Married \\
Separated \\
Divorced \\
\hline Widowed
\end{tabular}

Ethnicity:

White/Caucasian

Hispanic/Latino(a)

African-American/Black

Asian

Native American

Other - Please list:

What is your religious affiliation:

Christian - Protestant

Muslim 


Christian - Catholic
Hindu
Buddhist
Not religious

What is your family income?

Less than $\$ 10,000$

$\$ 10,000$ to $\$ 19,999$

$\$ 20,000$ to $\$ 29,999$

$\$ 30,000$ to $\$ 39,999$

$\$ 40,000$ to $\$ 49,999$

$\$ 50,000$ to $\$ 59,999$

$\$ 60,000$ to $\$ 69,999$

$\$ 70,000$ to $\$ 79,999$

$\$ 80,000$ to $\$ 89,999$

$\$ 90,000$ to $\$ 99,999$

$\$ 100,000$ to $\$ 149,999$

$\$ 150,000$ or more
Jewish

Atheist

Agnostic

Other - Please list:

How would you characterize your hometown? rural (unincorporated) small town (village or town) suburban (metropolitan area of a large city) small city (population $<30,000$ ) medium-sized city (population 30,000 - 100,000) large city (population $>100,000$ )

Please list all psychology courses that you have taken.

Are you color blind?

Yes
No

Do you have any specific phobias? (Please list all that apply)

1. Practiced meditation regularly $\mathrm{Y} / \mathrm{N}$

2. Practiced yoga regularly $\mathrm{Y} / \mathrm{N}$

3. Practiced Tai Chi regularly. Y/N

4. Practiced martial arts regularly $\mathrm{Y} / \mathrm{N}$ 


\section{State MAAS \\ (Brown and Ryan, 2003)}

Instructions: Using the 0-6 scale shown, please indicate to what degree you were having each experience described below when you were paged. Please answer according to what really reflected your experience rather than what you think your experience should have been.

$\begin{array}{ccccccc}0 & 1 & 2 & 3 & 4 & 5 & 6 \\ \text { not at all } & & & \text { somewhat } & & & \text { very much }\end{array}$

1. I was finding it difficult to stay focused on what was happening

2. I was doing something without paying attention.

3. I was preoccupied with the future or the past.

4. I was doing something automatically, without being aware of what I was doing.

5. I was rushing through something without being really attentive to it. 


\section{Mood Questionnaire \\ (Mata, Hogan, Joorman, Waugh \& Gibb, 2013)}

How positive are you feeling right now?

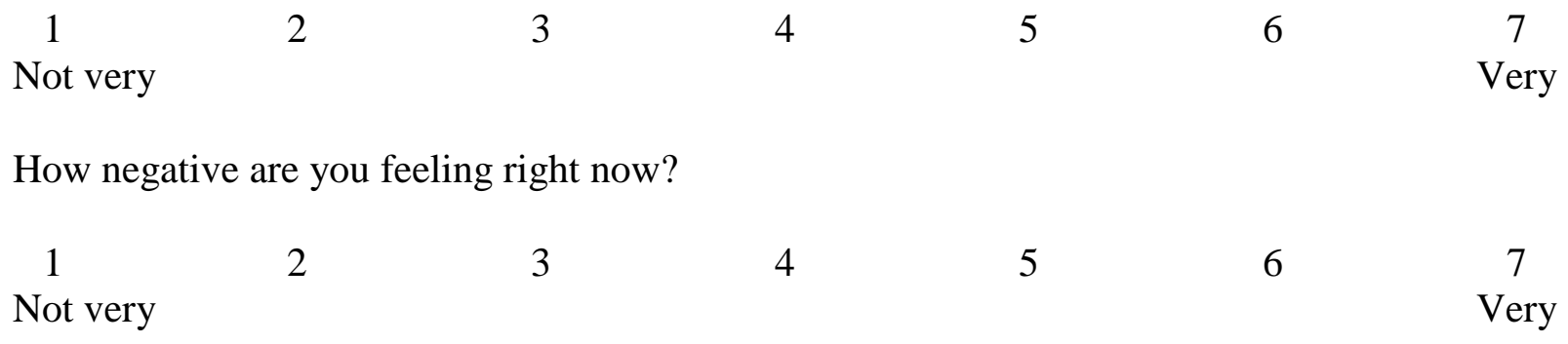

